SPECTROSCOPIC INVESTIGATION OF THE EFFECT OF POLYPHENOLIC COMPOUNDS ON THE AMYLOID- $\beta$ PROTEIN

\author{
A Dissertation \\ Presented to \\ The Doctoral Program Committee \\ At the University of Missouri-Columbia \\ In Partial Fulfillment \\ Of the Requirements for the Degree \\ Doctor of Philosophy \\ By \\ Brittany L. Hagenhoff \\ Dr. Renee JiJi, Dissertation Supervisor \\ December 2017
}


The undersigned, appointed by the dean of the Graduate School, have examined the dissertation entitled

\section{Spectroscopic Investigation of the Effect of Polyphenolic Compounds on the Amyloid- $\beta$ Protein}

Presented by Brittany L. Hagenhoff, a candidate for the degree of doctor of philosophy, and hereby certify that, in their opinion, it is worthy of acceptance.

Professor Renee D. JiJi

Professor C. Michael Greenlief

Professor Susan Z. Lever

Professor Gary A. Weisman 


\section{ACKNOWLEDGEMENTS}

I would never have gotten to this point without the guidance and patience

provided by my advisor, Dr. Renee JiJi, and for that I cannot thank her enough. Not only was she essential in facilitating my research goals but my teaching goals as well. She went above and beyond to help me pursue my passion for the classroom and gain invaluable experience.

I thank my committee members, Dr. Michael Greenlief, Dr. Susan Lever, and Dr. Gary Weisman for their input on my projects and enabling me to finish my work. I would like to express my gratitude to the Department of Chemistry at the University of Missouri-Columbia, which has not only provided the financial support and the means to conduct research, but also the opportunity to teach three different lecture courses that have helped me evolve as a young instructor in this field.

When I first joined Dr. JiJi's research group, I had no idea the impact the fellow graduate students would make on me. I have to thank all the members of the JiJi and Cooley research group, but particularly: Dr. Jian Xiong, Dr. Olayinka Oshokoya, and Dr. Michael Eagleburger. Their expertise and wisdom helped me more than they will know. I especially have to thank Dr. Anahita Zare and Bryan Lada. They have seen me through it all, good times and bad. They always showed their support and helped me more than I deserved.

Finally, thank you to my parents, Doug and Sandy Hagenhoff, and all my family and friends that have given me their love and support throughout my journey. I would not have made it here without them. 


\section{TABLE OF CONTENTS}

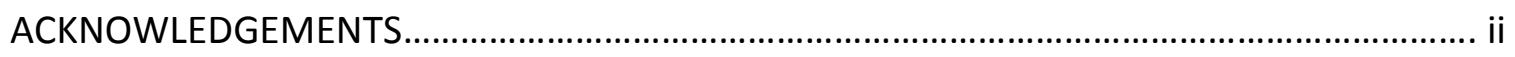

LIST OF FIGURES................................................................................................. vi

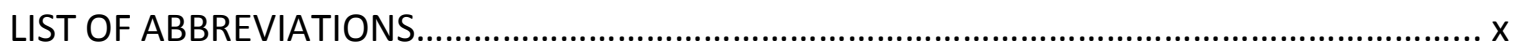

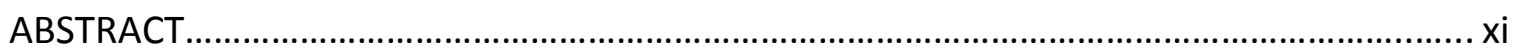

\section{CHAPTERS}

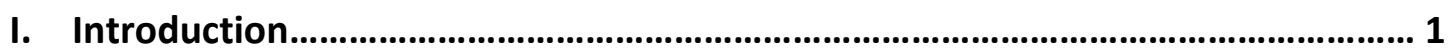

1.1 Alzheimer's Disease................................................................................... 1

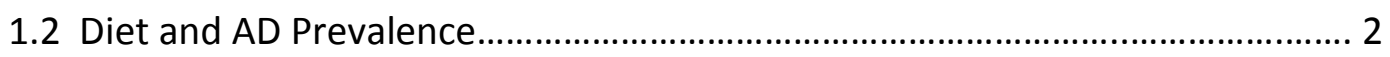

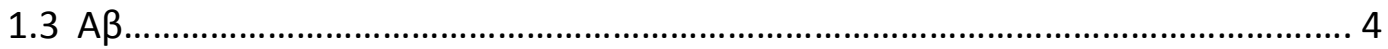

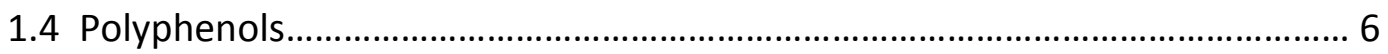

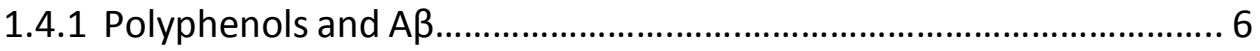

1.4.2 Structural Differences (Hydrophobicity vs Antioxidant Potency)...... 7

1.4.3 Polyphenol Structural Characteristics .................................................. 8

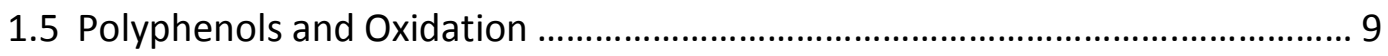

1.6 Spectroscopic Techniques Used..................................................................... 11

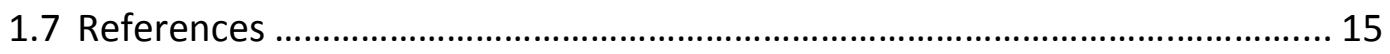

II. Increasing Ring Substituents Decreases $\beta$-sheet Formation.................................. 22

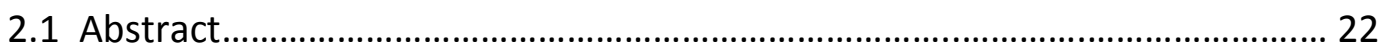

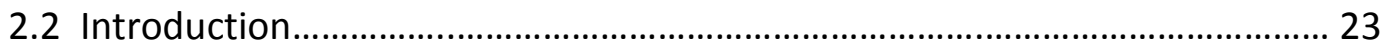

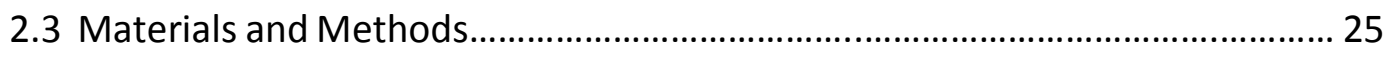

2.3.1 Materials ...................................................................................... 25

2.3.2 Amyloid- $\beta$ (1-42) Protein Preparation .............................................. 25

2.3.3 Circular Dichroism (CD) ............................................................... 26

2.3.4 Deep Ultraviolet Resonance Raman (dUVRR) .................................... 26

2.3.5 Data Analysis.................................................................................. 26

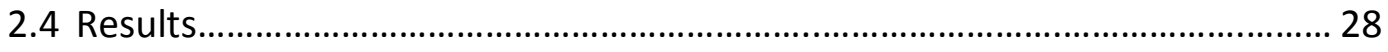




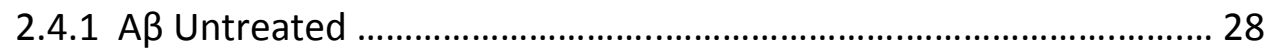

2.4.2 Piceatannol ..................................................................................... 29

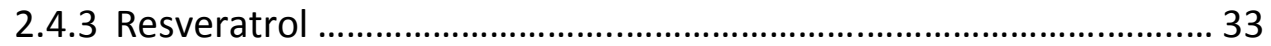

2.4.4 Diethylstilbestrol .......................................................................... 37

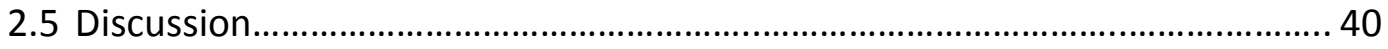

2.6 References............................................................................................ 43

III. Increasing Chain Linker Polarity Increases $\beta$-sheet Formation............................. 44

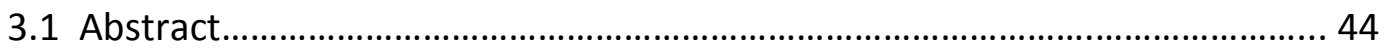

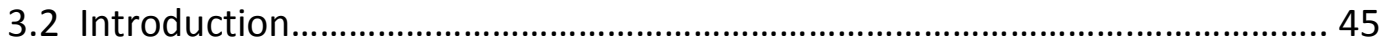

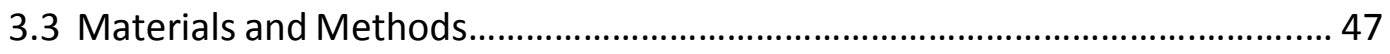

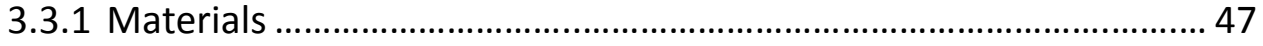

3.3.2 Spectroscopic Measurements and Data Analysis .............................. 47

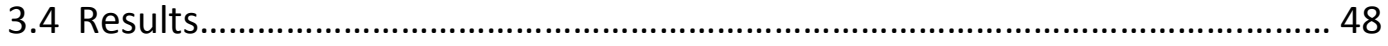

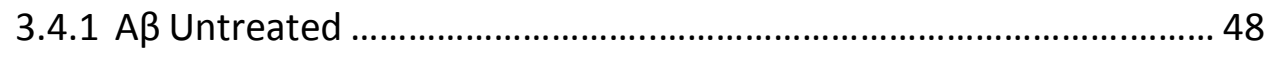

3.4.2 Nordihydroguaiaretic Acid ............................................................ 49

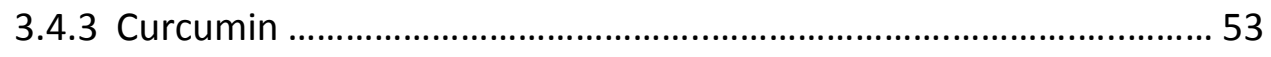

3.4.4 Rosmarinic Acid ........................................................................... 57

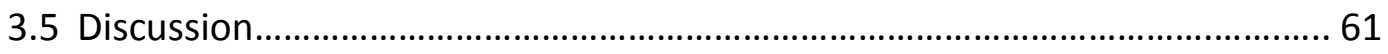

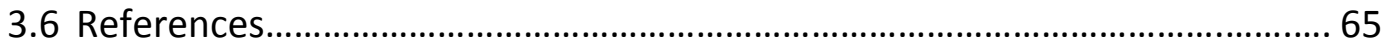

IV. Increasing Ring Substituents Destabilizes Pre-formed Amyloid- $\beta$ Fibrils............ 67

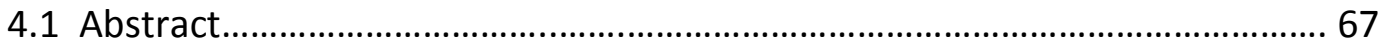

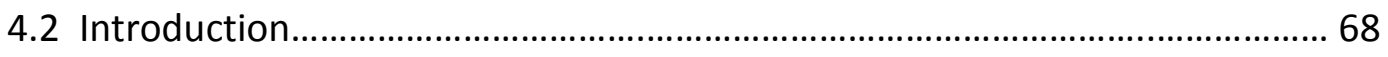

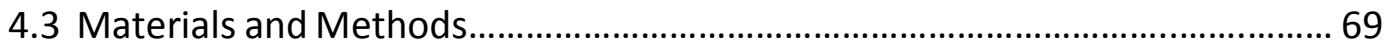

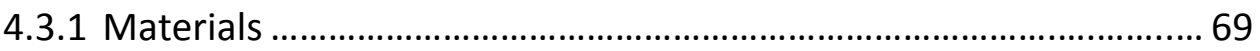

4.3.2 Amyloid- $\beta$ (1-42) Protein Preparation ................................................. 69

4.3.3 Circular Dichroism (CD) ................................................................. 70

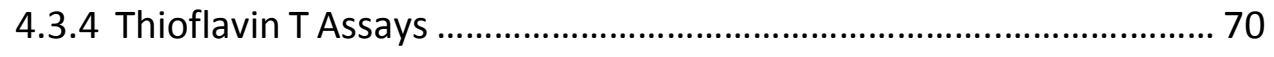

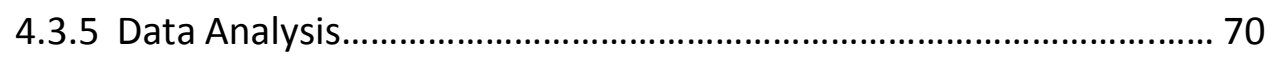




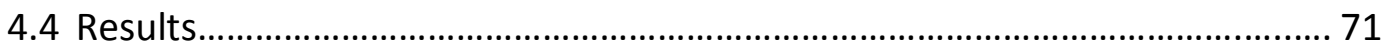

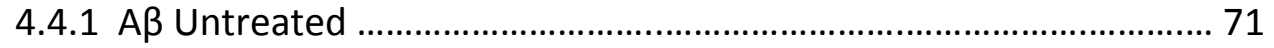

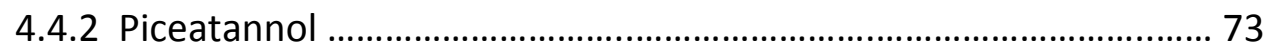

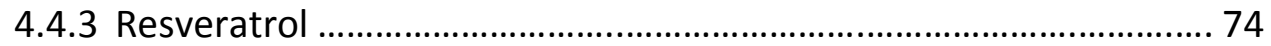

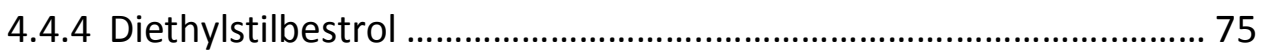

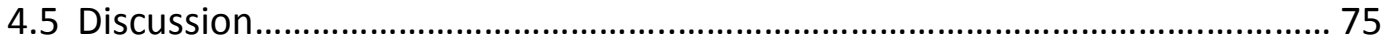

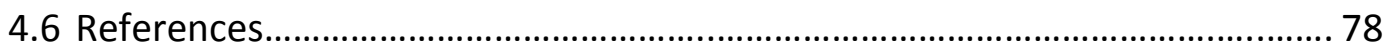

V. Increasing Chain Linker Polarity Destabilizes Pre-formed Amyloid- $\beta$ Fibrils...... 79

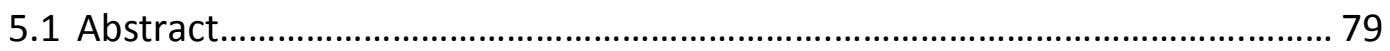

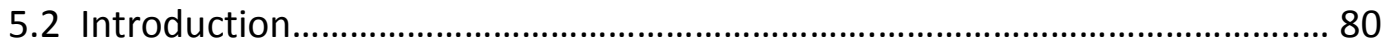

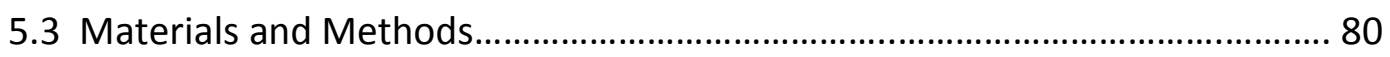

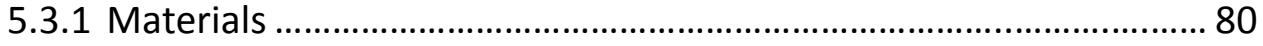

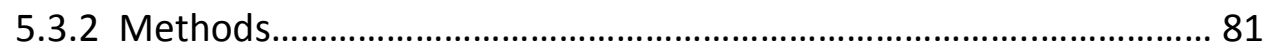

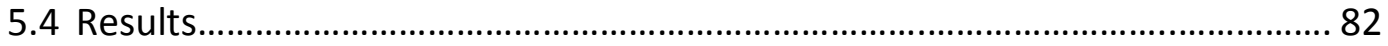

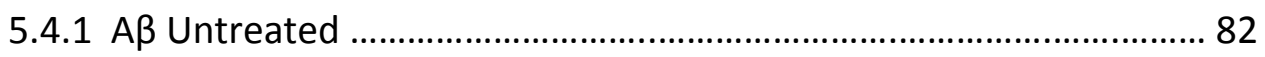

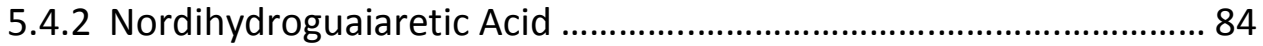

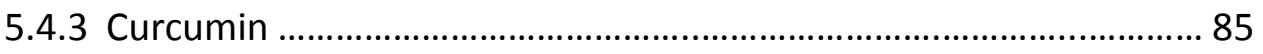

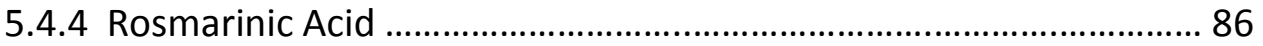

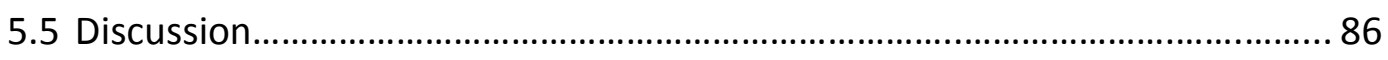

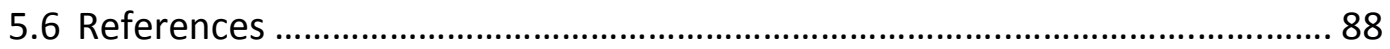

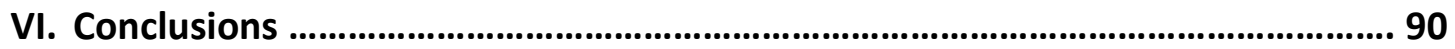

VITA 


\section{LIST OF FIGURES}

1.1 Prevalence and projection of Alzheimer's disease .................................................. 2

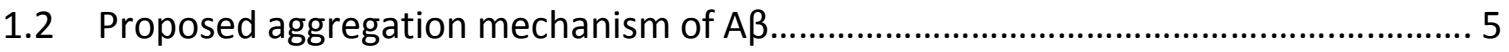

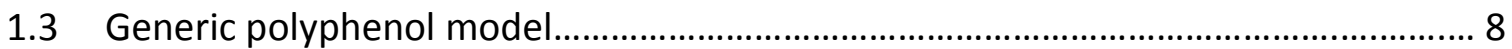

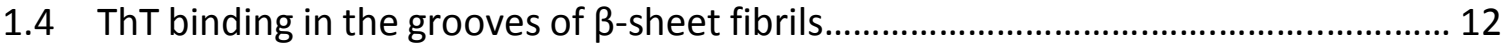

$1.5 C D$ spectra of secondary structure seen in $A \beta$ aggregation.................................... 13

1.6 Amide and aromatic modes present in a dUVRR spectrum of a protein.................. 14

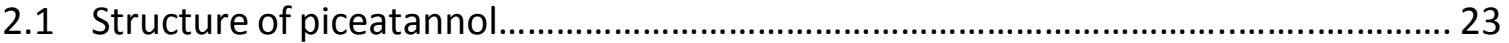

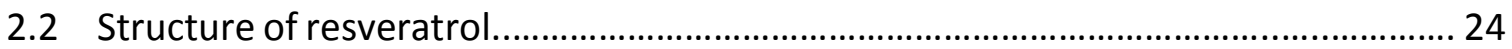

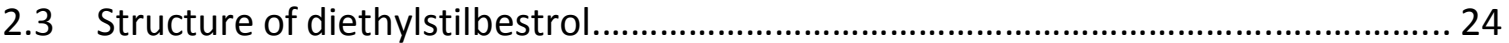

$2.4 \mathrm{CD}$ spectra of $\mathrm{A} \beta 42$ during a 12-day incubation period............................................. 28

2.5 CD spectra of $A \beta 42$ treated with piceatannol during a 12-day incubation period.. 29

2.6 Deconvoluted dUVRR spectra of A 342 treated with piceatannol on Days 0 and 12 30

2.7 DUVRR spectra of $A \beta 42$ overlaid with $A \beta 42$ treated with piceatannol on Day 0 and 12 31

2.8 DUVRR difference spectra of the deconvoluted peaks of $A \beta 42$ treated with

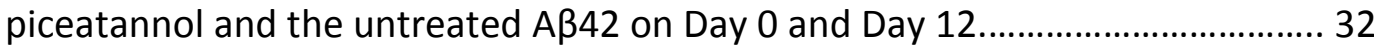

2.9 CD spectra of $A \beta 42$ treated with resveratrol during a 12-day incubation period.... 33

2.10 Deconvoluted dUVRR spectra of $A \beta 42$ treated with resveratrol on Days 0 and 12 34

2.11 DUVRR spectra of $A \beta 42$ overlaid with $A \beta 42$ treated with resveratrol on Days 0 and 12 35

2.12 DUVRR difference spectra of the deconvoluted peaks of $A \beta 42$ treated with resveratrol and the untreated $A \beta 42$ on Day 0 and Day 12 36

2.13 CD spectra of $A \beta 42$ treated with diethylstilbestrol during a 12-day incubation period 
2.14 Deconvoluted dUVRR spectra of $A \beta 42$ treated with diethylstilbestrol on Days 0

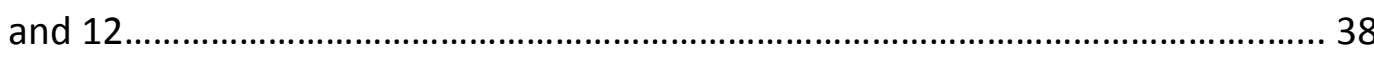

2.15 DUVRR spectra of $A \beta 42$ overlaid with $A \beta 42$ treated with diethylstilbestrol on Day 0 and 12

2.16 DUVRR difference spectra of the deconvoluted peaks of $A \beta 42$ treated with diethylstilbestrol and the untreated $A \beta 42$ on Days 0 and 12

2.17 Changes over the 12-day incubation period in the individual aromatic modes of the dUVRR spectra

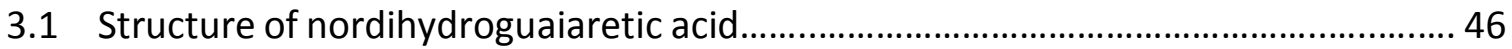

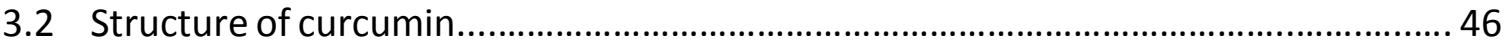

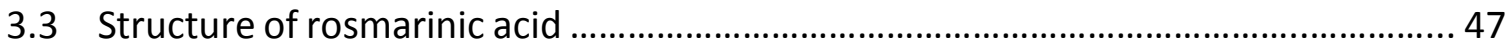

3.4 CD spectra of $A \beta 42$ during a 12-day incubation period............................................. 48

3.5 CD spectra of $A \beta 42$ treated with nordihydroguaiaretic acid during a 12-day incubation period.

3.6 Deconvoluted dUVRR spectra of $A \beta 42$ treated with nordihydroguaiaretic acid on Days 0 and 12 50

3.7 DUVRR spectra of $A \beta 42$ overlaid with $A \beta 42$ treated with nordihydroguaiaretic acid on Day 0 and 12 51

3.8 DUVRR difference spectra of the deconvoluted peaks of $A \beta 42$ treated with nordihydroguaiaretic acid and the untreated $A \beta 42$ on Day 0 and Day 12 52

3.9 CD spectra of $A \beta 42$ treated with curcumin during a 12-day incubation period....... 53

3.10 Deconvoluted dUVRR spectra $A \beta 42$ treated with curcumin on Days 0 and $12 \ldots \ldots . . .54$

3.11 DUVRR spectra of $A \beta 42$ overlaid with $A \beta 42$ treated with curcumin on Days 0 and 12 55

3.12 DUVRR difference spectra of the deconvoluted peaks of $A \beta 42$ treated with curcumin and the untreated $A \beta 42$ on Day 0 and Day 12 56

3.13 CD spectra of $A \beta 42$ treated with rosmarinic acid during a 12-day incubation period 
3.14 Deconvoluted dUVRR spectra A $\beta 42$ treated with rosmarinic acid on Days 0 and 12 58

3.15 DUVRR spectra of $A \beta 42$ overlaid with $A \beta 42$ treated with rosmarinic acid on Days 0 and 12 59

3.16 DUVRR difference spectra of the deconvoluted peaks of $A \beta 42$ treated with

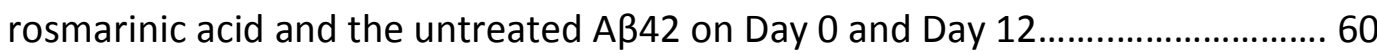

3.17 Blue shift of the amide $S$ mode of treated $A \beta 42$ 62

3.18 Changes over the 12-day incubation period in the individual aromatic modes of the dUVRR spectra 64

4.1 Structures of piceatannol, resveratrol, and diethylstilbestrol.................................69

4.2 CD spectra of $A \beta 42$ over a 12-day incubation period.............................................. 72

4.3 ThT assay of $A \beta 42$ over a 14-day incubation period.................................................. 72

4.4 CD spectra of $A \beta 42$ treated with piceatannol midway during a 12-day incubation period 73

4.5 CD spectra of $A \beta 42$ treated with resveratrol midway during a 12-day incubation period 74

4.6 CD spectra of $A \beta 42$ treated with diethylstilbestrol midway during a 12-day Incubation period...... 75

4.7 Intensity changes at $217 \mathrm{~nm}$ of the polyphenol-treated and untreated $A \beta$ samples over a 12-day incubation period 77

5.1 Structures of nordihydroguaiaretic acid, curcumin, and rosmarinic acid. 81

5.2 CD spectra of $A \beta 42$ over a 12-day incubation period. 82

5.3 ThT assay of $A \beta 42$ over a 14-day incubation period 83

5.4 CD spectra of $A \beta 42$ treated with nordihydroguaiaretic acid midway during a $12-$ day incubation period. 84

5.5 CD spectra of $A \beta 42$ treated with curcumin midway during a 12-day incubation period 
5.6 CD spectra of $A \beta$ treated with rosmarinic acid midway during a 12-day

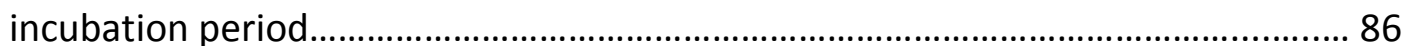




\section{LIST OF ABBREVIATIONS}

$\begin{array}{ll}\text { AD } & \text { Alzheimer's disease } \\ \text { A } & \text { Amyloid- } \beta \\ \text { APP } & \text { Amyloid Precursor Protein } \\ \text { NMR } & \text { Nuclear Magnetic Resonance } \\ \text { ROS } & \text { Reactive Oxygen Species } \\ \text { ThT } & \text { Thioflavin T } \\ \text { CD } & \text { Circular Dichroism } \\ \text { DUVRR } & \text { Deep Ultraviolet Resonance Raman } \\ \text { Y } & \text { Tyrosine } \\ \text { F } & \text { Phenylalanine }\end{array}$




\section{ABSTRACT \\ Spectroscopic Investigation of the Effect of Polyphenolic Compounds on the Amyloid- $\beta$ Protein \\ Brittany Hagenhoff}

Aggregation of the amyloid- $\beta(A \beta)$ protein is associated with the development of Alzheimer's disease. $A \beta$ is a 39-43 residue cleavage product of the amyloid precursor protein (APP). A $\beta$ aggregates to produce insoluble plaques in the brain, which are composed of cross $\beta$-sheet structured fibrils. Various polyphenolic compounds, both naturally occurring and synthetic, have been shown to interfere with $A \beta$ aggregation. To evaluate the ability of specific polyphenols to prevent $A \beta$ aggregation, this investigation utilized nordihydroguaiaretic acid, curcumin, rosmarinic acid, resveratrol, piceatannol, and diethylstilbestrol. These polyphenols differ in the number of ring substituents and the atom linker between the rings. The interaction of $A \beta$ with the polyphenolic compounds was analyzed using circular dichroism (CD) and deep ultraviolet resonance Raman (dUVRR) spectroscopies.

The polyphenols diethylstilbestrol, resveratrol, and piceatannol have increasing numbers of hydroxyl substituents on their rings, having two, three, and four respectively. It was found that with increasing number of hydroxyl ring substituents, the protein remained predominantly disordered and prevented formation of $\beta$-structure in the protein and aided in the destabilization of pre-formed $A \beta$ fibrils. Decreasing the number of hydroxyl substituents increases the likelihood of $\beta$-sheet formation, 
prevented destabilization of pre-formed $A \beta$ fibrils, and induced loss of stability of the protein.

The polyphenols nordihydroguaiaretic acid, curcumin, and rosmarinic acid have increasing polarity respectively in the chain linker between the phenolic rings. Each of these polyphenols have four ring substituents and have four to six atoms in their chain linker. It was found that with increasing the polarity of the linker, the protein had a greater tendency to form a $\beta$-structure, however pre-formed $A \beta$ fibrils were destabilized efficiently by all three polyphenols. Though a nonpolar chain linker pushed fibrillar protein toward a more disordered structure initially, the final state was similar regardless of added polyphenol. 


\section{Chapter I: Introduction}

\subsection{Alzheimer's Disease}

Alzheimer's disease $(A D)$ is a leading cause of dementia in people above the age of 65 and the sixth leading cause of death in the United States (Figure 1.1). AD is an irreversible neurodegenerative disease, and is characterized by synaptic failure, neuronal damage, and cellular degeneration in regions of the brain that mediate memory and cognitive function. ${ }^{[1,2,3]}$ Diagnosis of $A D$ is difficult as there is no single test that can diagnose the disease. AD accounts for about $60-80 \%$ of all diagnosed dementias with diagnosis made based upon clinical history, documentation of functional decline, and the ruling out of all other forms of dementia. A conformation of the diagnosis cannot be made until autopsy. ${ }^{[1,4]}$

There are two main hallmarks associated with $A D$ which are senile plaques and neurofibrillary tangles in the plasma and cerebrospinal fluid in the brain. Plaques, which are made up of the $A \beta$ protein, are believed to interfere with neuron communication at

synapses. ${ }^{[1,2]}$ Neurofibrillary tangles are made up of the tau protein and interfere with nutrient transport in neurons. ${ }^{[1-2,5-6]}$ 


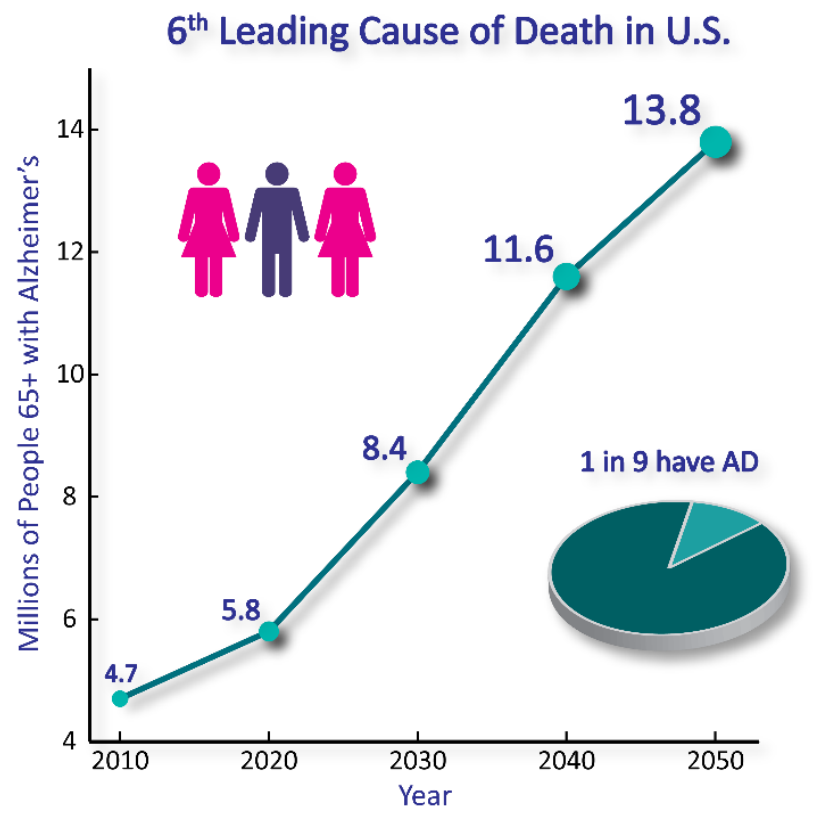

Figure 1.1: Diagnosis of Alzheimer's disease is on the rise, projected to reach nearly 14 million by year 2050. One in nine people over 65 years of age have the disease, with two out of every three of those being women. ${ }^{[1]}$

\subsection{Diet and AD Prevalence}

In 2010, there was an estimated 35.6 million people worldwide suffering with dementia with projections of over 65 million by $2030 .{ }^{[7]}$ There are many different forms of dementia including vascular dementia, Lewy body dementia, frontotemporal dementia, normal pressure hydrocephalus dementia, and mixed dementia, all with common outward symptoms such as cognitive impairment and memory loss with about $60-80 \%$ of these diagnoses being AD. ${ }^{[4]}$ The prevalence of dementia in 2010 was $2.6 \%$ in Africa, 3.9\% in Asia, 6.2\% in Europe, and 6.5\% in the Americas. ${ }^{[7]}$ Many ethnic groups living in the United States have much higher AD prevalence rates than those living in their homelands. ${ }^{[7]}$ Studies of these populations show that $6.24 \%$ of Nigerians living in the United States suffered from AD whereas only $1.41 \%$ of Nigerians suffered in Ibadan, 
Nigeria. In the United States, 4.1\% Japanese suffered from AD, while AD prevalence in Japan was only $1.88 \% .^{[8-10]}$

The prevalence of $A D$ among different ethnicities in the United States is roughly ranked as African Americans and Hispanics > American and European whites $>$ Asian Americans $>$ Native Americans. ${ }^{[1,7,11]}$ One of the primary differences among these ethnic groups is that they vary in location. A potentially unifying factor that could influence the chance of developing AD may be location and more specifically, diet. Different ethnicities and cultures have different eating and drinking habits, which could very well be one of the factors at the root of the differences in AD prevalence.

As one would assume, eating healthy should keep your overall health in good standing. The food pyramid is one representation of a healthy diet. However, which foods comprise the food pyramid varies depending on region. In general, high consumption of meat, butter, high-fat dairy products, eggs, refined sugar, saturated fatty acids, high caloric intake, and excessive alcohol consumption has a negative association with brain health, whereas diets rich in fruits, vegetables, fish, tea, coffee, and whole grains has a positive association. ${ }^{[12-13]}$ A diet that follows this general trend of high fresh food intake and low meat and fat intake is the Mediterranean diet. The Mediterranean diet consists of:[13-17]

- High intake of vegetables, legumes, fruits, nuts and seeds, potatoes, breads and cereals, and olive oil

- Moderate intake of fish 
- Low to moderate intake of dairy products (cheese and yogurt) from goat, sheep, buffalo, cow and camel

- Low intake of meat and poultry

- Low to moderate amount of alcohol wine

- Condiments, i.e., garlic, onion, and herbs

- Desserts of fresh fruit or sweets containing concentrated sugars or honey

Olive oil is the main source of fat, unlike in Western diets where animal fats are more common. Also, Western diets are higher in dairy products. ${ }^{[14-16]}$ The traditional Japanese diet is similar to the Mediterranean diet in that it is made up of mainly fish and plant foods, and is limited in animal fats. ${ }^{[31]}$ Conversely, the Western diet is comprised mostly of red and processed meats, refined grains, sweets, and moderate alcohol intake. ${ }^{[15,17]}$ These differences could be the reason that AD prevalence is higher in the U.S and Northern Europe than in the Mediterranean region and Japan.

Adherence to a healthy diet, much like the Mediterranean diet has been associated with reduced risk of cardiovascular disease and cancers, lowered rates of chronic diseases, reduced risk of neurodegenerative disease, reduced risk of $A D$, better cognitive functioning, and overall longer life expectancy. ${ }^{[13,18-21]}$

\subsection{Amyloid-6}

The buildup of the $A \beta$ protein into plaques is a hallmark of $A D .^{[1]} A \beta$ is a $\sim 4 \mathrm{kDa}$ protein that is $39-43$ residues long. It is produced from the proteolytic cleavage of the transmembrane amyloid precursor protein (APP) by $\beta$-secretase and $\psi$-secretase. ${ }^{[22-24]}$ 
Once $A \beta$ is cleaved from APP, the monomer can self-aggregate into various sized oligomers. ${ }^{[25-27]}$ The oligomers are transient in nature, changing size and structure quickly, making individual species difficult to isolate. The suggested mechanism in which the aggregation occurs (Figure 1.2) can differ for each oligomeric species as well. Unsystematic intermediates can form between monomers and oligomers before forming a fibril. ${ }^{[25-26,28-29]}$

One type of intermediate that can form is the protofibril. Protofibrils are short, flexible fibrils which are precursors of longer, more rigid fibrils. ${ }^{[28-29]}$ The occurrence of protofibrils decreases as fibril formation increases. ${ }^{[29]}$ The process of fibril formation by protofibril aggregation into mature fibrils is irreversible; they do not dissociate into protofibrils. ${ }^{[29]}$ All forms of $A \beta$ from monomers to high molecular weight oligomers have proven to be neurotoxic. ${ }^{[23]}$

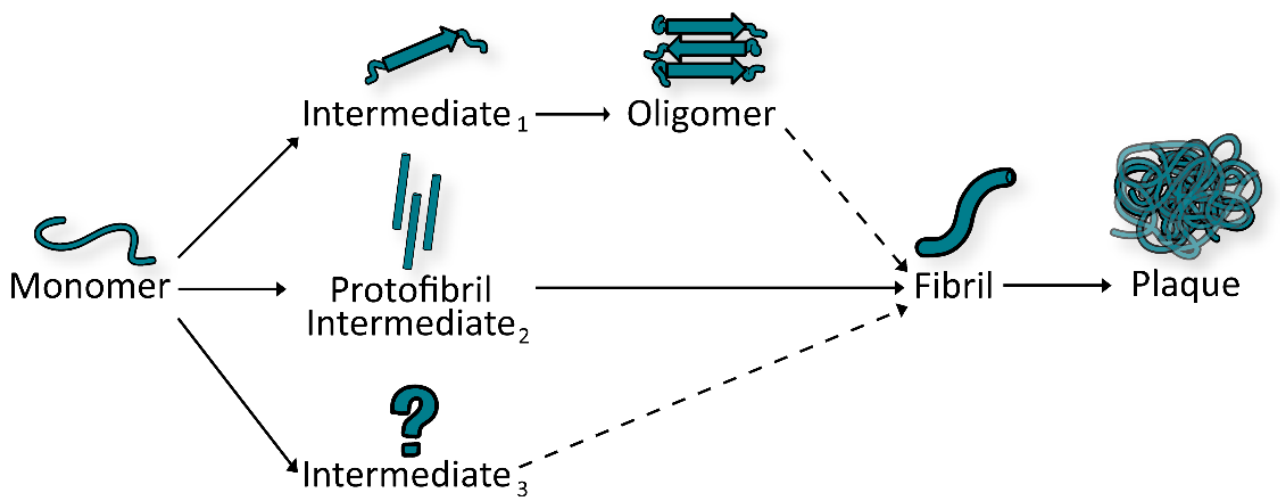

Figure 1.2: Proposed aggregation mechanism of AB.

The different species present in the aggregation pathway can take on different types of protein secondary structure. The $A \beta$ monomer is disordered in structure and as the protein begins to aggregate, it becomes more ordered forming $\beta$-sheet secondary structure. ${ }^{[25-26,28-29]}$ These secondary structures are based on the dihedral angles around 
$\mathrm{C}_{\alpha}$ of an amino acid in the protein backbone, phi and psi. ${ }^{[30]}$ The disordered secondary structure means there is no order, or more specifically, no one set of dihedral angles. The $\beta$-sheet secondary structure can be categorized into two types; parallel and antiparallel. Parallel $\beta$-strands align themselves in the same direction with carbonyl and amide groups staggered. Anti-parallel $\beta$-strands align themselves in opposite directions with carbonyl and amide groups lining up. Parallel $\beta$-sheets have phi and psi angles of $119^{\circ}$ and $113^{\circ}$, respectively, and anti-parallel $\beta$-sheets have phi and psi angles of $-139^{\circ}$ and $135^{\circ}$, respectively. ${ }^{[31]}$

\subsection{Polyphenols}

\subsubsection{Polyphenols and $A B$}

Polyphenols have been shown to reduce the toxicity of various forms of $A \beta$ and play a role in the $A \beta$ aggregation process. ${ }^{[32]}$ Structurally, polyphenols are aromatic rings with hydroxyl group substituents that are naturally found in plants and foods with over 8,000 compounds identified. ${ }^{[32-33]}$

As previously mentioned, $A \beta$ can self-aggregate in various ways and into various sized oligomers, changing in size and structure quickly. ${ }^{[25-29]}$ Although the exact mechanism is unknown, a general classification of polyphenols based on how they act on the aggregation of $A \beta$ can be made. The three classifications are those that prevent the formation of fibrils, those that prevent the extension of fibrils, and those that can destabilize pre-formed fibrils. ${ }^{[33,35]}$ 


\subsubsection{Structural Differences (Hydrophobicity vs Antioxidant Potency)}

Polyphenols vary widely in structure. They can be sub-classified into many groups based on structure, such as flavonoids, stilbenes, or lignans. The flavonoid group is the largest polyphenol group, with over 6,000 flavonoids identified, and has sparked research in many areas. ${ }^{[32-33,36]}$ All flavonoids have a similar $\left(\mathrm{C}_{6}-\mathrm{C}_{3}-\mathrm{C}_{6}\right)$ structure with two aromatic rings bound by a 3-carbon formed oxygenated heterocycle which makes up a third ring, and differ mainly in the substituents on the rings. ${ }^{[37]}$ Stilbenes have a similar structure to flavonoids except for one less carbon that prevents the third ring from forming (i.e., $\mathrm{C}_{6}-\mathrm{C}_{2}-\mathrm{C}_{6}$ ). Instead, the two rings are connected by a double bond; i.e., methylene bridge. ${ }^{[32,37]}$ Lignans are a combination of two phenylpropane units where two $C_{6}-C_{3}$ structures are attached at their central $\left(C_{8}\right)$ carbon. ${ }^{[32-33,38]}$ Some polyphenols do not fall into a specific classification and are grouped as an "other polyphenol".

Flavonoids have been heavily researched due to their vast abundance. However, the focus of this research is on non-flavonoid polyphenols such as stilbenes and other polyphenols. Depending on the classification of the polyphenol, different structural characteristics are present. In this investigation, the polyphenols used were each linear polyphenols (non-flavonoid) each with two rings, a chain linker between the rings, and hydroxyl group substituents on the rings.

Polyphenols can act upon any stage of the $A \beta$ aggregation process, with certain structural characteristics suiting early or late stages more appropriately. Recent studies have surfaced that deal with the structural significance of polyphenols. ${ }^{[34,38-39]}$ Certain 
characteristics that can play a role on how it will behave are the number and type of substituents on the rings, length of the atom linker between the rings, and the polarity of the atom linker. An example of a generic polyphenol is shown in Figure 1.3.

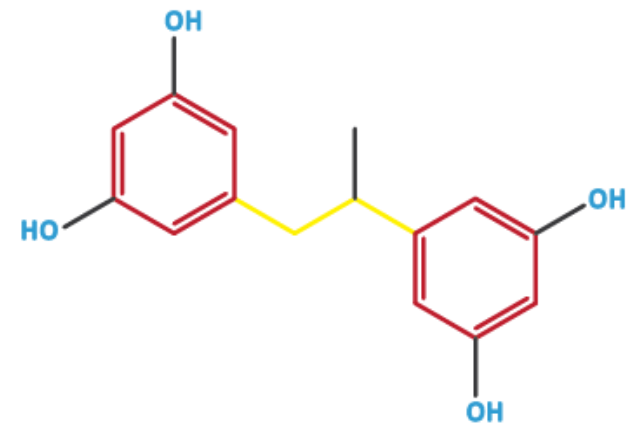

Figure 1.3: Generic polyphenol model: hydroxyl group substituents (blue), aromatic rings (red), and an atom linker chain (yellow).

\subsubsection{Polyphenol Structural Characteristics}

Hydrophobicity and antioxidant potency of polyphenols have been shown to affect their ability to inhibit $A \beta$ aggregation. It is believed that hydrophobicity interferes with the formation and extension stages, whereas antioxidative potency affects the fibrillization stages. ${ }^{[34]}$ Two rings with a minimum of two to three hydroxyl substitutions on those rings is preferred. ${ }^{[34]}$ Polyphenols can interact with proteins through a multitude of ways, including aromatic $\pi-\pi$ stacking interactions between the polyphenolic rings and aromatic residues, hydrogen bonding between the hydroxyl substituents and the protein backbone, and hydrophobic interactions. ${ }^{[34,40-45]}$

Structurally, a polyphenol with both high hydrophobicity and antioxidant potency would be the most desirable for preventing $A \beta$ aggregation. This would allow binding to hydrophobic motifs of the protein, interference with aromatic stacking that could facilitate aggregation, formation of hydrogen bond which could weaken salt bridges between aggregating $A \beta$ species, and provide free radical scavenging. ${ }^{[34]}$ 
Binding of each individual polyphenol to the $A \beta$ protein not only depends on the polyphenol's structure but also which $A \beta$ species it is binding to along the aggregation pathway. Disordered monomers versus small oligomers versus $\beta$-sheet fibrils will bind polyphenols by different means due to their variation of secondary and higher order structure. Typically, polyphenols interact with the peptide at residues such as tyrosine10 and phenylalanines- 4,19 , and 20 due to $\pi$-stacking interactions of the aromatic residues with the phenolic rings of the polyphenol..$^{[46-47]}$

Isolated segments of the protein change in secondary structure differently. For example, the C-terminus has a higher probability of rapid oligomerization due to the increased number of nonpolar residues. ${ }^{[46]}$ Lührs et al. showed two parallel $\beta$-sheets that run along the fibril axis from residues $\approx 18-26$ and $\approx 31-42$ through the use of hydrogen/deuterium-exchange nuclear magnetic resonance (NMR) spectroscopy. ${ }^{[48]}$ Polyphenols interacting with residues within this region could prevent the $\beta$-strands from developing. Polyphenols such as curcumin have been shown to interact with residues in this region including phenylalanines-19 and 20, serine-26, isoleucine-32, and methionine-35 as well as within the loop of these $\beta$-strands at lysine-28. ${ }^{[47-48]}$ Once $\beta$ strands have formed, polyphenols such as rosmarinic acid and resveratrol, have been shown to hydrogen bond along the side of $\beta$-sheet amyloid structures promoting destabilization of fibrils. ${ }^{[47,49]}$

\subsection{Polyphenols and Oxidation}

Polyphenols are famous for their antioxidative properties and are abundant in fruits, vegetables, and beverages, such as tea and red wine. Free radicals are products 
of naturally occurring processes in cells, which in turn cause oxidative stress in the cells that can lead to cell death. Antioxidants remove free radicals by scavenging them. ${ }^{[50]}$ Polyphenols have been known to possess hepatoxic, antioxidant, anti-inflammatory, antimicrobial, anticancer, antiviral, anti-allergic, and anti-ulcer effects. ${ }^{[33]}$ Polyphenols have many different biological actions, such as interaction with transition metals, inactivation of free radicals, precipitation of protein, inhibition of inflammatory response, modulation of the activity of different enzymes, and effects on intracellular signaling pathways and gene expression. ${ }^{[51-54]}$

Reactive oxygen species (ROS) are free radicals with a single unpaired electron that are produced during naturally occurring cellular processes that can damage proteins, nucleic acids, and lipid membranes leading to membrane instability eventually causing apoptosis. ${ }^{[55-56]}$ Oxidative stress occurs when the generation of ROS is higher than the level of antioxidants acting against them. Examples of ROS are superoxide anions $\left(\mathrm{O}_{2}{ }^{--}\right)$, hydroxyl radicals $(\mathrm{OH})$, and hydrogen peroxide $\left(\mathrm{H}_{2} \mathrm{O}_{2}\right) \cdot{ }^{[57]}$ Increasing polyphenol intake can combat ROS generation due to the ability of polyphenols to donate a hydrogen atom to lipids and other molecules, thereby preventing their oxidation by ROS. ${ }^{[54]}$

Protein oxidation and increased oxidative stress has been shown in numerous studies to lead to the manifestations of $A D .^{[57-58]}$ The brain is particularly susceptible to free radical damage due to a high content of easily peroxidizable lipids, a high rate of oxygen consumption, and a lack of antioxidants in relation to other organs in the body. ${ }^{[2,58-59]}$ Protein oxidation impairs protein synthesis, neuronal function, enzyme 
activation, and the ability of cells to regulate homeostasis leading to cognitive decline. ${ }^{[59-60]}$

ROS produce oxidative stress which induces intracellular $A \beta$ accumulation in cell cultures and $A \beta$ itself has been shown to induce oxidative stress in vivo and in vitro. ${ }^{[36,62-}$

66] Studies have shown that lipid peroxidation is enhanced by increases in intracellular $A \beta .{ }^{[63]}$ The oxidation of the methionine- 35 residue in $A \beta$ could play an important role in free radical and oxidative stress generation. ${ }^{[2,63,65]}$ The tyrosine-10 residue is also an easy target of oxidation in the protein. ${ }^{[2,63]}$ Studies by Murakami et al. have shown that the oxidation of tyrosine-10 could be lead to oxidation of the methionine- 35 residue, thereby contributing to the increase in free radical concentration and neurotoxicity of the protein. ${ }^{[63]}$ Thus, a vicious cycle between ROS and $A \beta$ accumulation may accelerate the progression of AD.

\subsection{Spectroscopic Techniques Used}

Spectroscopic techniques are frequently utilized for analysis of biological samples, such as proteins. Different forms of spectroscopy instrumentation were implemented throughout this study. For $A \beta$, Thioflavin $T$ (ThT) assays are commonly used to monitor fibril formation. In order to observe the secondary structure, two different forms of spectroscopy are commonly used, circular dichroism (CD) and deep ultraviolet resonance Raman (dUVRR).

The ThT assay is a fluorescence based method that can monitor the formation of $A \beta$ fibrils in solution over time. The fluorophore selectively binds to $\beta$-sheet fibrils 
(Figure 1.4) causing it to fluoresce. ${ }^{[67]}$ The assay confirms the presence of cross- $\beta$-sheet structure, which is only present in amyloid fibrils.

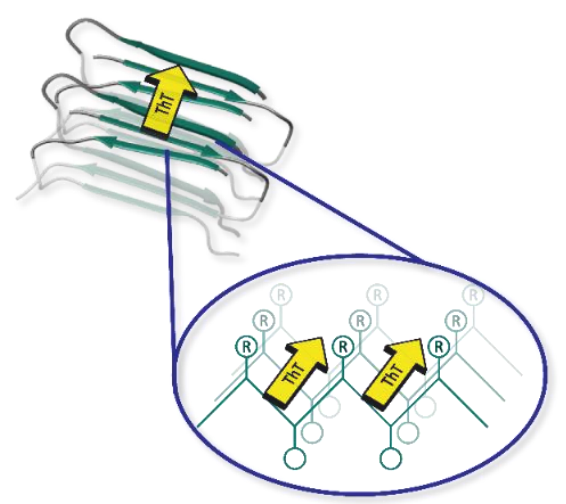

Figure 1.4: ThT binds in the grooves of B-sheet fibrils increasing fluorescence.

CD involves illuminating samples with circularly polarized light and then measuring the difference in absorption of left and right polarized light. ${ }^{[68]}$ Depending on the secondary structure of the protein and the dihedral angles that correspond with the structure, the light will be absorbed differently producing characteristic CD spectra as seen in Figure 1.5. If the protein is lacking defined structure and is disordered, a strong minimum at $197 \mathrm{~nm}$ and a slight maximum at $217 \mathrm{~nm}$ will be observed. For a $\beta$-sheet structured protein, the spectrum is nearly flipped with a strong minimum at $217 \mathrm{~nm}$ and a slight maximum at $197 \mathrm{~nm}$. From this, classification of secondary structure was obtained as well as the changes in the structure over time. 


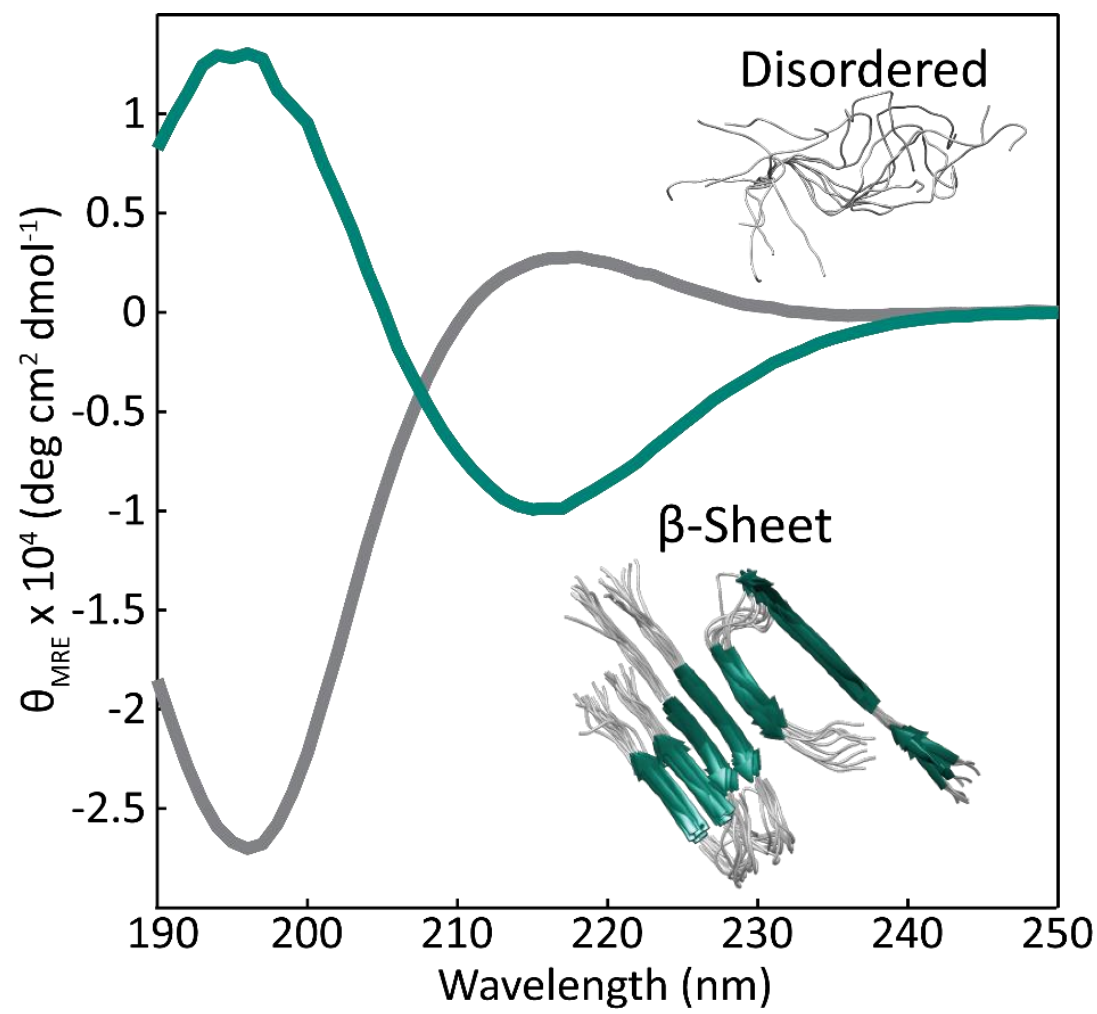

Figure 1.5: CD spectra of secondary structure seen in AB aggregation; disordered structure (grey) with a negative minimum at $197 \mathrm{~nm}$ and a slight positive maximum at $217 \mathrm{~nm}$, and b-sheet (teal) with a positive minimum at $197 \mathrm{~nm}$ and a negative minimum at $217 \mathrm{~nm}$.

DUVRR is an emerging technique for the determination of secondary structure changes of a protein. Raman is an inelastic scattering method in which the emitted light from molecules is shifted to different frequencies than the incident excitation light. ${ }^{[69]}$ With dUVRR, the laser excites within the deep UV region and enhances the vibrational modes of the amide backbone, as well as aromatic side chains. ${ }^{[70]}$ DUVRR spectra of proteins have four protein backbone associated amide bonds; amide I ( $\mathrm{C}=\mathrm{O}$ stretching) at $1650-1680 \mathrm{~cm}^{-1}$, amide II (C-H/N-H in phase stretching/bending) at $1480-1560 \mathrm{~cm}^{-1}$, amide III (C-H/N-H out of phase stretching/bending) at $1240-1315 \mathrm{~cm}^{-1}$, and amide S (coupled $\mathrm{C}-\mathrm{H} / \mathrm{N}-\mathrm{H}$ bending) at $1350-1400 \mathrm{~cm}^{-1}$, as seen in Figure 1.6. ${ }^{[71]}$ Intensity changes in these regions signify changes in secondary structure. When a protein 
changes from disordered to $\beta$-sheet, there are not typically high intensity changes in the amide modes. There can be shift in the amide $\mathrm{S}$ mode when transitioning between these two structures as well as slight intensity changes in amide I. There can also be changes in the environment of the aromatic residues of the protein. $A \beta$ contains tyrosine $(Y)$ and phenylalanine (F). The Y1 tyrosine modes are at 820 and $858 \mathrm{~cm}^{-1}$ and the $Y 9 \mathrm{a}, \mathrm{Y} 7 \mathrm{a}$, and Y8b tyrosine modes are present at 1191, 1213, and $1608 \mathrm{~cm}^{-1}$, respectively. ${ }^{[72]}$ The phenylalanine modes are represented by the $\mathrm{F} 12$ mode at $1008 \mathrm{~cm}^{-1}, \mathrm{~F}$ mode at 1191 and $1213 \mathrm{~cm}^{-1}$, and F8b and F8a modes at 1586 and $1608 \mathrm{~cm}^{-1}$, respectively. ${ }^{[72]}$ With the use of these different spectroscopic techniques, the impact of polyphenols on the aggregation of $A \beta$ can be determined.

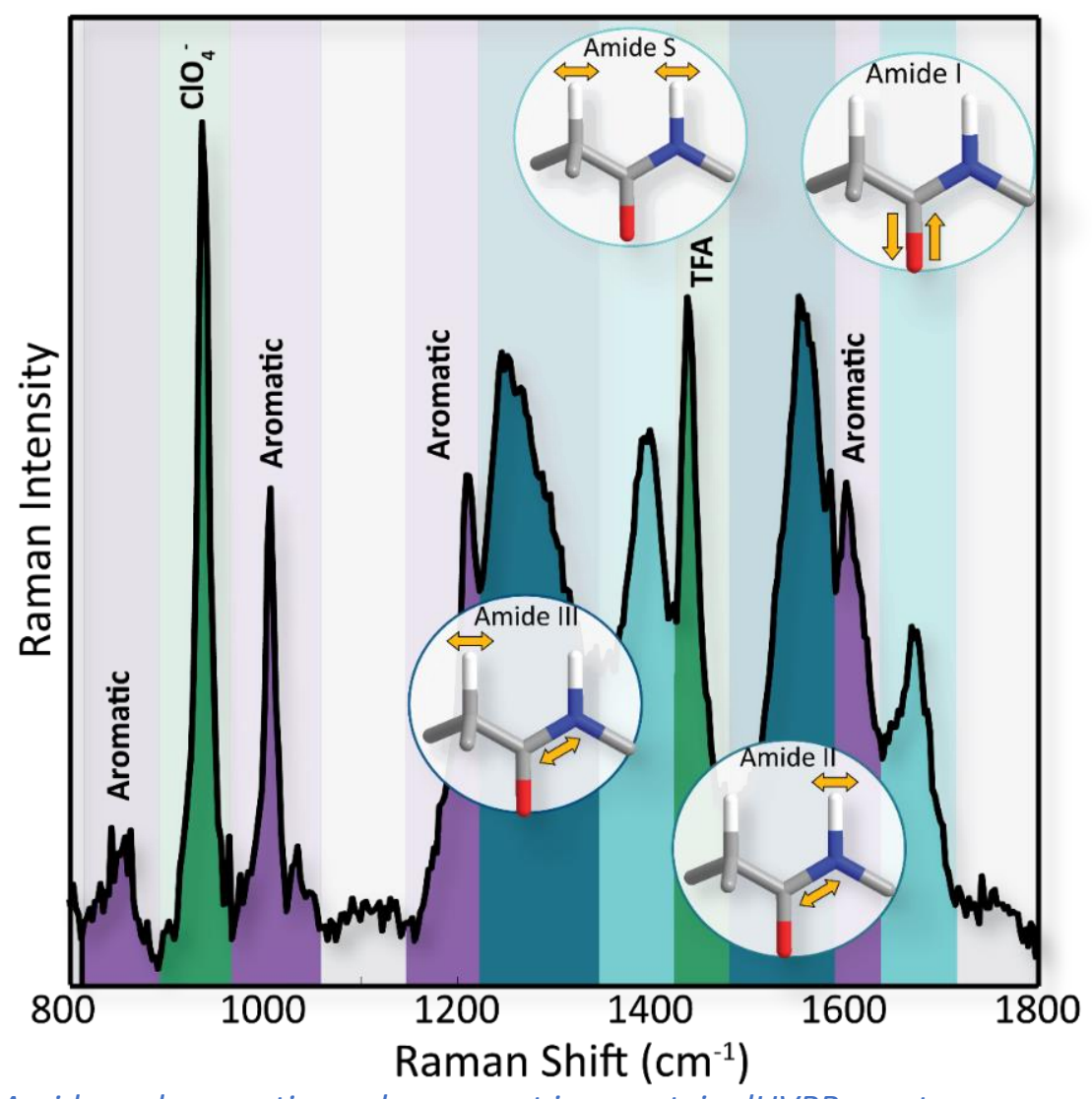

Figure 1.6: Amide and aromatic modes present in a protein dUVRR spectrum. 


\subsection{References}

1. Alzheimer's ssocation. 2014 Alzheimer's disease facts and figures. Alzheimer's \& Dementia 2014, $10(2), 4-28$.

2. Masters, C. L.; Cappai, R.; Barnham, K. J.; Villemagne, V. L., Molecular mechanisms for Alzheimer's disease: implications for neuroimaging and therapeutics. J Neurochem 2006, 97 (6), 1700-25.

3. Pike, C. J. B., D.; Walencewicz, A. J.; Glabe, C. G.; Cotman, C. W., Neurodegeneration Induced by $\beta$-Amyloid peptides in vitro: the role of peptide assembly state. J Neurosci 1993, 13 (4), 1676 1687.

4. Neugroschl, J.; Wang, S. Alzheimer's disease: Diagnosis and treatment across the spectrum of disease severity. Mt Sinai J Med 2012, 78 (4), 596-612.

5. Alzheimer's Disease.

http://nihseniorhealth.gov/alzheimersdisease/whatisalzheimersdisease/01.html.

6. Haass, C. S., M. G.; Hung, A. Y.; Vigo-Pelfrey, C.; Mellon, A.; Ostaszewski, B. L.; Lieberburg, I.; Koo, E. H.; Schenk, D.; Teplow, D. B.; Selkoe, D. J. , Amyloid-beta peptide is produced by cultured cells during normal metabolism. Nature 1992, 359, 322-325.

7. World Alzheimer Report - Executive Summary. Alzheimer's Disease 2009, 6-8.

8. White, L.; Petrovitch, H.; Ross, G. W.; Masaki, K. H.; Abbott, R. D.; Teng, E. L.; Rodriguez, B. L.; Blanchette, P. L.; Havlik, R. J.; Wergowske, G.; Chiu, D.; Foley, D. J.; Murdaugh, C.; Curb, D., Prevalence of dementia in older Japanese-American men in Hawaii. JAMA 1996, 276 (12), 955960.

9. Hendrie, H. C.; Osuntokun, B. O.; Hall, K. S., Prevalence of Alzheimer's disease and dementia in two communities: Nigerian Africans and African Americans. Am J Psychiatry 1995, 152 (10), 14851492. 
10. Graves, A. B.; Larson, E. B.; Edland, S. D.; Bowen, J. D.; McCormick, W. C.; McCurry, S. M.; Rice, M. M.; Wenzlow, A.; Uomoto, J. M., Prevalence of dementia and its subtypes in the Japanese American population of King County, Washington State. Am J Epidemiol 1996, 144 (8), 760-771.

11. Mehta, K. M.; Yeo, G. W., Systematic review of dementia prevalence and incidence in US race/ethnic populations. Alzheimers Dement 2017, 13, 72-83.

12. Morris, M. C.; Evans, D. A.; Bienias, J. L.; Tangney, C. C.; Bennett, D. A.; Wilson, R. S.; Aggarwal, N.; Schneider, J., Consumption of fish and n-3 fatty acids and risk of incident Alzheimer disease. Arch Neurol 2003, 60, 940-946.

13. Hu, N.; Yu, J. T.; Tan, L.; Wang, Y. L.; Sun, L.; Tan, L., Nutrition and the risk of Alzheimer's disease. Biomed Res Int 2013, 2013, 1155-1167.

14. Gillette-Guyonnet, S.; Vellas, B., Diet and prevention of cognitive decline and dementia. Canadian Review of Alzheimer's Disease and Other Dementias 2007, September, 18-27.

15. Willet, W. C.; Sacks, F.; Trichopoulou, A.; Drescher, G.; Perro-Luzzi, A.; Helsing, E.; Trichopoulou, D., Mediterranean diet pyramid: a cultural model for healthy eating. Am J Clin Nutr 1995, 61, 1402S-1406S.

16. Kromhout, D.; Keys, A.; Aravanis, C.; Buzina, R.; Fidanza, F.; Giampaoli, S.; Jansen, A.; Menotti, A.; Ndeljkovic, S.; Pekkarinen, M.; Simic, B. S.; Toshima, H., Food consumption patterns in the 1960s in seven countries. Am J Clin Nutr 1989, 49, 889-894.

17. Allbaugh, L. G., Crete: a case study of an underdeveloped area. Princeton University Press: Princeton, NJ, 1953.

18. Kesse-Guyot, E.; Andreeva, V. A.; Jeandel, C.; Ferry, M.; Hercberg, S.; Galan, P., A healthy dietary pattern at midlife is associated with subsequent cognitive performance. J Nutr 2012, 142 (5), 909-915.

19. Hankey, G. J., Nutrition and the risk of stroke. The Lancet Neurology 2012, 11 (1), 66-81.

20. Feart, C. S., C.; Rondeau, V.; Amieva, H.; Portet, F.; Dartigues, JF.; Scarmeas, N.; BarbergerGateau, P., Adherence to a Mediterranean diet, cognitive decline, and risk of dementia. JAMA 2009, 302 (6), 638-648. 
21. Willet, W. C., Diet and Health: What Should We Eat? Science 1994, 264, 532-537.

22. Kang, J.; Lemaire, H. G.; Unterbeck, A.; Salbaum, J. M.; Masters, C. L.; Grzeschik, K. H.; Multhaup, G.; Beyreuther, K.; Muller-Hill, B., The precursor of Alzheimer's disease amyloid A4 protein resembles a cell-surface receptor. Nature 1987, 325 (6106), 733-736.

23. Cappai, R. W., A. R. , Amyloid $\beta$. Int Biochem \& Cell Bio 1999, 31 (9), 885-889.

24. Glenner, G. G.; Wong, C. W., Alzheimer's disease: initial report of the purificaiton and characterization of a novel cerebrovascular amyloid protein. Biochem Biophys Res Commun 1984, $16(120), 885-890$.

25. Ono, K.; Li, L.; Takamura, Y.; Yoshiike, Y.; Zhu, L.; Han, F.; Mao, X.; Ikeda, T.; Takasaki, J.; Nishijo, H.; Takashima, A.; Teplow, D. B.; Zagorski, M. G.; Yamada, M., Phenolic compounds prevent amyloid beta-protein oligomerization and synaptic dysfunction by site-specific binding. J Biol Chem 2012, 287 (18), 14631-43.

26. Necula, M.; Kayed, R.; Milton, S.; Glabe, C. G., Small molecule inhibitors of aggregation indicate that amyloid beta oligomerization and fibrillization pathways are independent and distinct. J Biol Chem 2007, 282 (14), 10311-10324.

27. Roychaudhuri, R.; Yang, M.; Deshpande, A.; Cole, G. M.; Frautschy, S.; Lomakin, A.; Benedek, G. B.; Teplow, D. B., C-terminal turn stability determines assembly differences between Abeta40 and Abeta42. J Mol Biol 2013, 425 (2), 292-308.

28. Walsh, D. M.; Lomakin, A.; Benedek, G. B.; Condron, M. M.; Teplow, D. B., Amyloid B-Protein Fibrillogenesis. J Biol Chem 1997, 272, 22364-22372.

29. Harper, J. D.; Wong, S. S.; Lieber, C. M.; Lansbury, P. T., Observation of metastable AB amyloid protofibrils by atomic force microscopy. Chem Biol 1997, 4, 119-125.

30. Ramachandran, G. N.; Ramakrishnan, C.; Sasisekharan, V. Stereochemistry of polypeptide chain configurations. J Mol Biol 1963, 7, 95-99.

31. Pauling, L.; Corey, R. B., The pleated sheet, a new layer configuration of polypeptide chains. Proc Natl Acad Sci 1951, 37, 251-256. 
32. Lakey-Beitia, J.; Berrocal, R.; Rao, K. S.; Durant, A. A., Polyphenols as therapeutic molecules in Alzheimer's disease through modulating amyloid pathways. Mol Neurobiol 2015, 51 (2), 466-479.

33. Odeh, F.; Al-Jaber, H.; Khater, D., Nanoflora - How nanotechnology enhanced the use of active phytochemicals. Application of Nanotechnology in Drug Delivery 2014, Chapter 10, 344-346.

34. Shoval, H.; Lichtenberg, D.; Gazit, E., The molecular mechanisms of the anti-amyloid effects of phenols. Amyloid 2007, 14 (1), 73-87.

35. Ono, K.; Yoshiike, Y.; Takashima, A.; Hasegawa, K.; Naiki, H.; Yamada, M., Potent antiamyloidogenic and fibril-destabilizing effects of polyphenols in vitro: implications for the prevention and therapeutics of Alzheimer's disease. J Neurochem 2003, 87 (1), 172-181.

36. Harnly, J. M.; Doherty, R. F.; Beecher, G. R.; Holden, J. M.; Haytowitz, D. B.; Bhagwat, S.; Gebhardt, S., Flavonoid content of U.S. fruits, vegetables, and nuts. J Agric Food Chem 2006, 54, 9966-9977.

37. Li, F. G., Q.; Dong, H.; Shi, J., Resveratrol, a neuroprotective supplement for Alzheimer's disease. Curr Pharm Des 2012, 18, 27-33.

38. Manach, C. S., A.; Morand, C.; Remesy, C.; Jimenez, L., Polyphenols: food sources and bioavailability. Am J Clin Nutr 2004, 79, 727-747.

39. Churches, Q. I.; Caine, J.; Cavanagh, K.; Epa, V. C.; Waddington, L.; Tranberg, C. E.; Meyer, A. G.; Varghese, J. N.; Streltsov, V.; Duggan, P. J., Naturally occurring polyphenolic inhibitors of amyloid beta aggregation. Bioorg Med Chem Lett 2014, 24 (14), 3108-3112.

40. Porat, Y.; Abramowitz, A.; Gazit, E., Inhibition of amyloid fibril formation by polyphenols: structural similarity and aromatic interactions as a common inhibition mechanism. Chem Biol Drug Des 2006, 67 (1), 27-37.

41. Gazit, E., Mechanisms of amyloid fibril self-assembly and inhibition: Model short peptides as a key research tool. FEBS J 2005, 272, 5971-5978.

42. Gazit, E., Global analysis of tandem aromatic octapeptide repeats: The significance of the aromatic-glycine motif. Bioinformatics 2002, 18 (6), 880-883. 
43. Porat, Y.; Mazor, Y.; Efrat, S.; Gazit, E., Inhibition of islet amyloid polypeptide fibril formation: a potential role for heteroaromatic interactions. Biochemistry 2004, 43, 14454-14462.

44. Azriel, R.; Gazit, E., Analysis of the minimuml amyloid-forming fragment of the islet amyloid polypeptide. An experimental support for the key role of the phenylalanine residue in amyloid formation. J Biol Chem 2001, 276 (36), 34156-34161.

45. Hudson, S. A.; Ecroyd, H.; Dehle, F. C.; Musgrave, I. F.; Carver, J. A., (-)-Epigallocatechin-3-gallate (EGCG) maintains kappa-casein in its pre-fibrillar state without redirecting its aggregation pathway. J Mol Biol 2009, 392 (3), 689-700.

46. Serpell, L. C. Alzheimer's amyloid fibrils: structure and assembly. BBA - Mol Basis Dis 2000, 1502 (1), 16-30.

47. Wang, Y.; Latshaw, D. C.; Hall, C. K. Aggregation of $A \beta(17-36)$ in the presence of naturally occuring phenolic inhibitiors using coarse-grained simulations. J Mol Bio 2017, In-press. [Online early access]. http://www.sciencedirect.com/science/article/pii/s0022283617304886.

48. Lührs, T.; Ritter, C.; Adrian, M.; Riek-Loher, D.; Bohrmann, B.; Döbeli, H.; Schubert, D.; Riek, R. 3D structure of Alzheimer's amyloid- $\beta$ (1-42) fibrils. PNAS 2005, 102 (48), 17342-17347.

49. Ramazzotti, M.; Melani, F.; Marchi, L.; Mulinacci, N.; Gestri, S.; Tiribilli, B.; Degl'Innocenti, D. Mechanisms for the inhibition of amyliod aggregation by small ligands. Biosci Rep 2016, 36 (5), 114.

50. Choi, D. Y.; Lee, Y. J.; Hong, J. T.; Lee, H. J., Antioxidant properties of natural polyphenols and their therapeutic potentials for Alzheimer's disease. Brain Res Bull 2012, 87 (2-3), 144-153.

51. Obrenovich, M. E.; Nair, N. G.; Beyaz, A.; Aliev, G.; Reddy, V. P., The role of polyphenolic antioxidants in health, disease, and aging. Rejuventation Res 2010, 13 (6), 631-643.

52. Rojanathammanee, L.; Puig, K. L.; Combs, C. K., Pomegranate polyphenols and extract inhibit nuclear factor of activated T-cell activity and microglial activation in vitro and in a transgenic mouse model of Alzheimer disease. J Nutr 2013, 143 (5), 597-605.

53. Bravo, L., Polyphenols: chemistry, dietary sources, metabolism, and nutritional significance. Nutritions Reviews 1998, 56 (11), 317-333. 
54. Sun, A. Y.; Wang, Q.; Simonyi, A.; Sun, G. Y., Botanical phenolics and brain health. Neuromolecular Med 2008, 10 (4), 259-274.

55. Romano, A. D.; Serviddio, G.; de Matthaeis, A.; Bellanti, F.; Vendemiale, G., Oxidative stress and aging. J Nephrol 2010, 23 (S15), S29-S36.

56. Nunomura, A.; Castellani, R. J.; Zhu, X.; Moreira, P. I.; Perry, G.; Smith, M. A., Involvement of oxidative stress in Alzheimer disease. J Neuropathol Exp Neurol 2006, 65 (7), 631-641.

57. Keller, J. N.; Schmitt, F. A.; Scheff, S. W.; Ding, Q.; Chen, Q.; Butterfield, D. A.; Markesbery, W. R., Evidence of increased oxidative damage in subjects with mild cognitive impairment. Neurology 2005, 64, 1152-1156.

58. Floyd, R. A.; Hensley, K., Oxidative stress in brain aging - Implications for therapeutics of neurodegenerative diseases. Neurobio Aging 2002, 23, 795-807.

59. Mattson, M. P.; Chan, S. L.; Duan, W., Modification of brain aging and neurodegenerative disorders by genes, diet, and behavior. Physiol Rev 2002, 82, 637-672.

60. Ding, Q.; Markesbery, W. R.; Chen, Q.; Li, F.; Keller, J. N., Ribosome dysfunction is an early event in Alzheimer's disease. J Neurosci 2005, 25 (40), 9171-9175.

61. Berlett, B. S.; Stadtman, E. R. Protein oxidation in aging, disease, and oxidative stress. J Bio Chem 1997, 272, 20313-20316.

62. Rinaldi, P.; Polidori, M. C.; Metastasio, A.; Mariani, E.; Mattioli, P.; Cherubini, A.; Catani, M.; Cecchetti, R.; Senin, U.; Mecocci, P., Plasma antioxidants are similarly depleted in mild cognitive impairment and in Alzheimer's disease. Neurobiology of Aging 2003, 24 (7), 915-919.

63. Misonou, H.; Marishima-Kawashima, M.; Ihara, Y., Oxidative stress induces intracellular accumulation of Amyloid $\beta$-protein $(A \beta)$ in human neuroblastoma cells. Biochemistry 2000, 39, 6951-6959.

64. Paola, D.; Domenicotti, C.; Nitti, M.; Vitali, A.; Borghi, R.; Cottalasso, D.; Zaccheo, D.; Odetti, P.; Strocchi, P.; Marinari, U. M.; Tabaton, M.; Pronzato, M. A., Oxidative stress induces increase in intracellular amyloid beta-protein production and selective activation of betal and betall PKCs in NT2 cells. Biochem Biophys Res Commun 2000, 268 (2), 642-646. 
65. Murakami, K.; Irie, K.; Ohigashi, H.; Hara, H.; Nagao, M.; Shimizu, T.; Shirasawa, T., Formation and stabilization model of the 42-mer $A \beta$ radical - Implications for the long-lasting oxidative stress in Alzheimer's disease. J Am Chem Soc 2005, 127, 15168-15174.

66. Tabner, B. J.; El-Agnaf, O. M.; Turnbull, S.; German, M. J.; Paleologou, K. E.; Hayashi, Y.; Cooper, L. J.; Fullwood, N. J.; Allsop, D., Hydrogen peroxide is generated during the very early stages of aggregation of the amyloid peptides implicated in Alzheimer disease and familial British dementia. J Biol Chem 2005, 280 (43), 35789-35792.

67. Schoneich, C., Methionine oxidation by reactive oxygen species: reaction mechanisms and relevance to Alzheimer's disease. Biochim Biophys Acta 2005, 1703 (2), 111-119.

68. Coalier, K. A.; Paranjape, G. S.; Karki, S.; Nichols, M. R. Stability of early-stage amyloid- $\beta$ (1-42) aggregation species. BBA 2013, 1834, 65-70.

69. Greenfield, N. J. Analysis of circular dichroism data. Methods in Enzymology 2004, 383, 282-317.

70. Mikhonin, A. V. Direct UV resonance Raman monitoring of protein folding reaction coordinate: alpha-helix melting and formation revisited. Ph. D Thesis, University of Pittsburgh, 2006.

71. Wang, Y.; Purrello, R.; Jordan, T.; Spiro, T. G. UVRR spectroscopy of the peptide bond. 1. Amide S, a nonhelical structure marker, is a $\mathrm{C}_{\alpha} \mathrm{H}$ bending mode. J Am Chem Soc 1991, 113, 6359-6368.

72. Austin, J. C.; Jordan, T.; Spiro, T. G. Ultraviolet resonance Raman studies of proteins and related model compounds. Chapter 2. Biomolecular Spectroscopy, Part A. Clark, R. J. H.; Hester, R. E. John Wiley \& Sons Ltd. 1993; 55-127. 


\section{Chapter II: Increasing Ring Substituents Decreases $\beta$-sheet Formation}

\subsection{Abstract}

Stilbenoids have common structural components consisting of two phenolic rings with hydroxyl group substituents and a chain linker between the rings. Hydroxyl group substituents on the polyphenolic rings not only facilitate hydrogen bonding with the protein backbone but also provide antioxidant properties. The antioxidant capacity increases with the number of substituents. This study investigated the ability of stilbenoids, differing in the number of hydroxyl substituents to prevent $\beta$-sheet fibril formation. The polyphenols diethylstilbestrol, resveratrol, and piceatannol are structurally similar, differing in the number of hydroxyl group substituents, having two, three, and four, respectively. It was found that with increasing number of hydroxyl ring substituents on the polyphenols, the protein remained more disordered and formed smaller amounts of $\beta$-structure. Diethylstilbestrol, with only two ring substituents, did not prevent formation of $\beta$-sheet structure, which could in turn lead to the formation of fibrillar plaques in the brain. 


\subsection{Introduction}

Hydroxyl group substituents on polyphenolic rings allow for essential binding to the proteins through hydrogen bonding of the polyphenol to the amide rich backbone of the proteins. ${ }^{[1-3]}$ Studies have shown that greater numbers of hydroxyl groups on polyphenols, is correlated with higher the antioxidant activity. ${ }^{[4]}$ Ono et al. compared four flavonoid compounds (myricetin, morin, quercetin, and kaempferol ) that have similar structures and differ only in the numbers of hydroxyl substituents. It was found that decreasing the number of hydroxyl substituents decreased the anti-amyloidogenic and fibril-destabilizing activity of the polyphenol. ${ }^{[4]}$

This study sought to determine the effect of the number of hydroxyl substituents on stilbenoids compounds, on their ability to prevent $\beta$-sheet fibril formation. The polyphenols diethylstilbestrol, resveratrol, and piceatannol have two, three, and four hydroxyl substituents on their rings, respectively. They each have a 2-atom carbon chain between the two rings allowing for a conjugated double bond system in the molecule.

Piceatannol is a naturally occurring stilbenoids found in plants such as Japanese knotweed, as well as in fruits including many grape families. ${ }^{[5]}$ Figure 2.1 shows the structure of the first polyphenol analyzed, piceatannol.

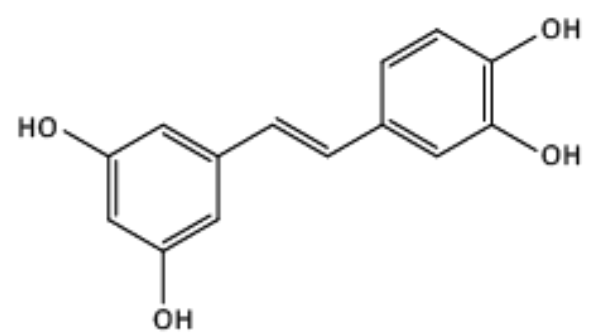

Figure 2.1: Structure of piceatannol. 
Resveratrol is found in many fruits, such as grapes, blueberries, and cranberries, and is famous for its presence in red wines. ${ }^{[6]}$ Resveratrol, shown in Figure 2.2, has one less hydroxyl group substituent than piceatannol resulting in a diminished antioxidant potency.

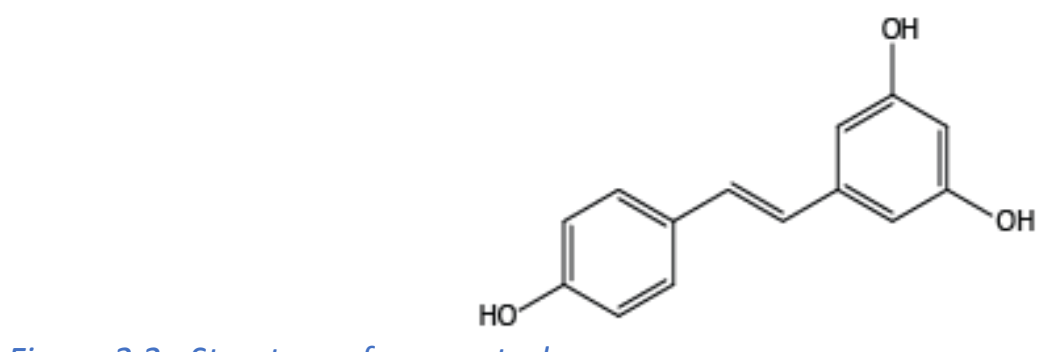

Figure 2.2: Structure of resveratrol.

Diethylstilbestrol was first synthesized to mimic estrogen and was prescribed to pregnant woman in the mid-1900's to aid in pregnancy complications. ${ }^{[7]}$ In the early 1970's, diethylstilbestrol was found to be an endocrine disruptor, classified as a carcinogen, and ultimately prohibited. ${ }^{[7]}$ Diethylstilbestrol, shown in Figure 2.3 , has the least number of hydroxyl substituents of the three compounds with only two, one on each ring, further lessening its antioxidant potency.

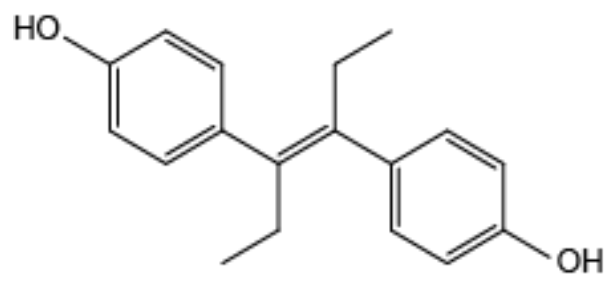

\section{Figure 2.3: Structure of diethylstilbestrol.}

The aim of this study was to investigate how the number of hydroxyl group substituents on stilbenoids affects the aggregation of $A \beta$. 


\subsection{Materials and Methods}

\subsubsection{Materials}

$\mathrm{A} \beta(1-42)$ (>95\% pure) was purchased from Atlantic Peptides (Scarborough, ME). Ethanol and sodium perchlorate were purchased from Sigma-Aldrich (St. Louis, MO) and used without further purification. Sodium phosphate monobasic, sodium phosphate dibasic, sodium chloride, and cyclohexane were purchased from Fisher Scientific (Pittsburgh, PA). Resveratrol, piceatannol, and diethylstilbestrol were purchased from Sigma-Aldrich (St. Louis, MO).

\subsubsection{Amyloid- $\beta$ (1-42) Protein Preparation}

$A \beta$ (1-42) (M.W. $4512.3 \mathrm{kDa}$ ) was dissolved in $10 \mathrm{mM}$ sodium phosphate buffer, $20 \mathrm{mM} \mathrm{NaCl}, \mathrm{pH} 7.4$, sonicated for $10 \mathrm{~min}$ in an ice bath and centrifuged for 30 minutes at $14,000 \mathrm{rcf}$ and $4^{\circ} \mathrm{C}$ (Centrifuge $5804 \mathrm{R}$, Eppendorf AG, Hamburg Germany). The concentration was estimated using the extinction coefficient of tyrosine, which is 1,280 $\mathrm{M}^{-1} \mathrm{~cm}^{-1}$ at $280 \mathrm{~nm}$ using a Varian Cary Bio 50 UV-Vis spectrophotometer (Varian, Palo Alto, $C A)^{[8]}$. Peptide concentrations ranged from $70-80 \mu \mathrm{M}$ and were normalized in all the spectral processing. $A \beta$ was prepared and incubated at $37^{\circ} \mathrm{C}$ over a 12 -day time period. During this time, samples were analyzed every four days by CD and on Day 0 and Day 12 by dUVRR spectroscopy. Stock solutions of each polyphenolic compound were prepared in ethanol at a concentration of $5 \mathrm{mM}$. Polyphenolic compounds were then added to the protein solution for a final concentration of $25 \mu \mathrm{M}$. For dUVRR spectroscopy, sodium perchlorate was added to the sample as an internal intensity standard at a final concentration of $50 \mathrm{mM}$. 


\subsubsection{Circular Dichroism (CD)}

A Jasco J-815 CD Spectropolarimeter (Jasco, Easton, MD) was used to collect CD spectra in the range of $190-250 \mathrm{~nm}$. A scan speed of $50 \mathrm{~nm} / \mathrm{min}$ with a response time of $4 \mathrm{sec}$ was used. Five spectra were averaged for each sample. A $1 \mathrm{~mm}$ optical path length quartz cuvette (Hellman, Plainview, NY) was employed for all measurements.

\subsubsection{Deep Ultraviolet Resonance Raman (dUVRR)}

The dUVRR instrument has been described previously by Wang et al. ${ }^{[8]}$ Briefly, UV light was generated using the fourth harmonic of a tunable $4 \mathrm{kHz}$ frequency quadrupled Ti:Sapphire laser (Coherent Inc., Santa Clara, CA), which was pumped by a diode-pumped frequency doubled Nd:YLF laser (Coherent Inc., Santa Clara, CA). An excitation wavelength of $197 \mathrm{~nm}$ was employed for all measurements and the average power at the sample was attenuated to c.a. $500 \mu \mathrm{W}$ to prevent sample degradation. The excitation light was directed towards the sample stream guided by two Nitinol wires. The sample was kept under $\mathrm{N}_{2}$ gas to eliminated ambient oxygen, as $\mathrm{O}_{2}$ has a feature at $1550 \mathrm{~cm}^{-1}$. Spectra were calibrated using a cyclohexane spectrum and $50 \mathrm{mM}$ sodium perchlorate was used as an internal intensity standard.

\subsubsection{Data Analysis}

Data analysis was carried out in the MATLAB R2016b (MathWorks, Natick, MA) environment. For all dUVRR spectra, the background buffer spectrum was subtracted and cosmic spikes were removed using an in-house cosmic ray removal algorithm. A nonlinear least-squares (NLLS) algorithm was used to fit each spectrum with a series of 
Gaussian/Lorentzian bands, which approximate the Voigt line shape, as described previously. ${ }^{[9]}$ All dUVRR spectra are the average of triplicate trials.

The amide I mode was deconvoluted into two peaks at 1657 and $1670 \mathrm{~cm}^{-1}$. The amide II mode was centered at $1559 \mathrm{~cm}^{-1}$, and the amide S mode was centered 1400 $\mathrm{cm}^{-1}$. The amide III mode was deconvoluted into three bands at 1244, 1275, and 1311 $\mathrm{cm}^{-1} . A \beta$ has one tyrosine and three phenylalanines. It is likely that the tyrosine mode at $1263 \mathrm{~cm}^{-1}$ also contributes to the intensity of the amide III bands. Other tyrosine modes that show up in the dUVRR spectrum of $A \beta$ include the fermi doublet (Y1) fitted at 820 and $858 \mathrm{~cm}^{-1}$. The band at $820 \mathrm{~cm}^{-1}$ is about $10 \mathrm{~cm}^{-1}$ lower than expected, which is likely due to the high noise level at this region. The fitted peak at $1191 \mathrm{~cm}^{-1}$ is an overlapping phenylalanine and tyrosine mode (F/Y9a) that arises from $\mathrm{C}-\mathrm{H}$ bending. The ring stretching modes of phenylalanine and tyrosine are also overlapped with combined bonds at 1213 and $1608 \mathrm{~cm}^{-1}$. The feature at $936 \mathrm{~cm}^{-1}$ is from perchlorate, which was added as an internal intensity standard. A sharp feature at $1441 \mathrm{~cm}^{-1}$ is present in some spectra because TFA was added as a counter ion during the purification of early batches of the synthetic $A \beta$. The counter ion was later replaced by hydrochloride to remove this peak from the spectra. Difference spectra were created by subtraction of dUVRR spectra of $A \beta$ from spectra of samples treated with polyphenols at each time point (Days 0 and 12). The difference spectra were multiplied by ten to better visualize the differences. Further difference spectra were created by subtraction of Day 0 dUVRR spectra from Day 12 dUVRR spectra to visualize the structural impact of the polyphenols made on the protein over time in the aromatic regions. 
All CD spectra were averaged and the background buffer spectrum was

subtracted from the raw data. The mean residue ellipticity $\left(\theta_{\mathrm{MRE}}\right)$ was calculated using Equation 1 with $\vartheta$ being the raw ellipticity from the measurement (degrees), $M W$ being the molecular weight of the protein $(\mathrm{g} / \mathrm{mol}), n$ being the number of protein residues, $d$ being the path length of the cell $(\mathrm{cm})$, and $C$ being the concentration of the sample (M). ${ }^{[8-10]}$ All spectra are averaged spectra of triplicate trials.

Equation 1:

$$
\left[\theta_{M R E}\right]=\frac{\theta \cdot M W}{10 \cdot n \cdot d \cdot C}
$$

\subsection{Results}

\subsubsection{AB Untreated}

Figure 2.4 shows the $C D$ spectra of $A \beta$ during the 12-day incubation period.

Initially on Day 0 , the protein is disordered in structure, as indicated by the minimum at $197 \mathrm{~nm}$. Over time, this feature decreases in intensity and another minimum is formed at $217 \mathrm{~nm}$, indicative of $\beta$-sheet formation.

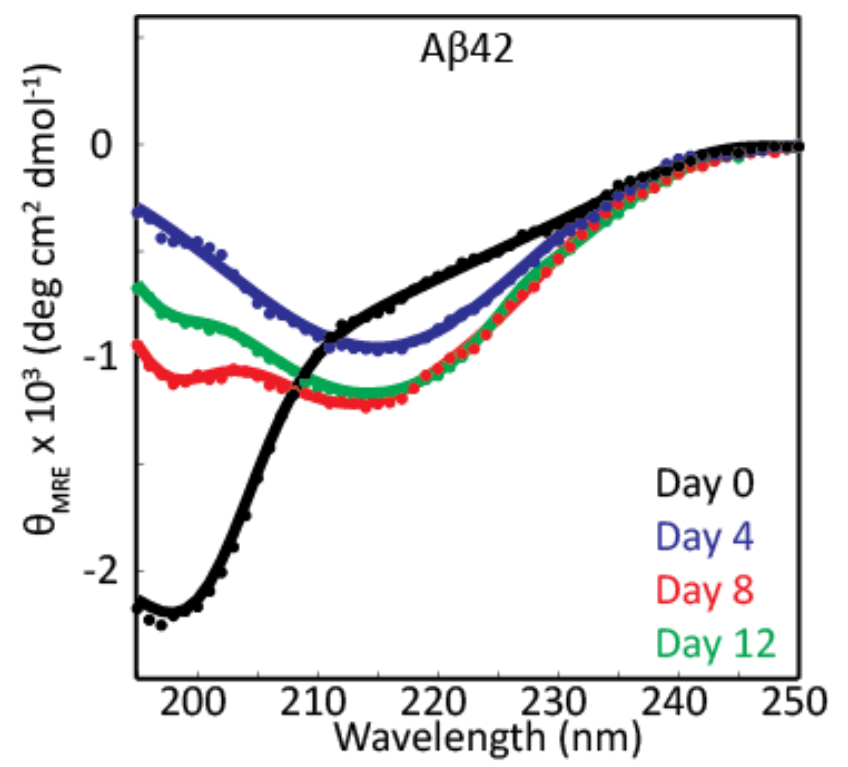

Figure 2.4: CD spectra of AB42 during a 12-day incubation period; Day 0 (black), Day 4 (blue), Day 8 (red), and Day 12 (green). 


\subsubsection{Piceatannol}

Figure 2.5 shows the $C D$ spectra of $A \beta$ treated with piceatannol during the $12-$ day incubation period. Initially on Day 0 , the protein is disordered in structure, as indicated by the minimum at $197 \mathrm{~nm}$. There is a slight loss of intensity at $197 \mathrm{~nm}$ on Day 4 but over time, the protein remains in a disordered state with no additional changes in structure. Thus, the addition of piceatannol prevents the protein from forming $\beta$-sheet structure.

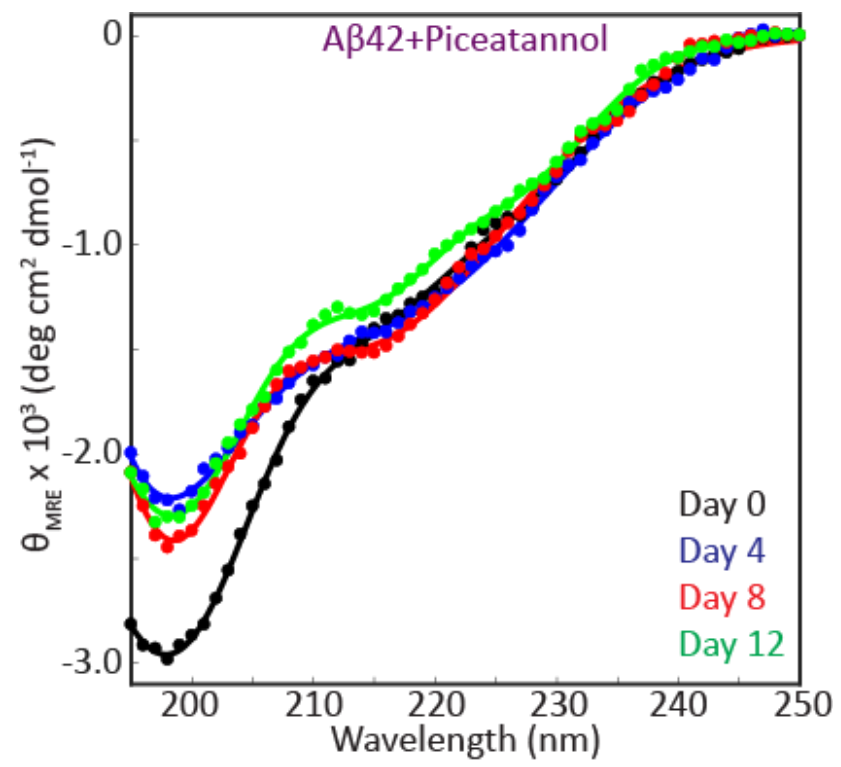

Figure 2.5: CD spectra of AB42 treated with piceatannol during a 12-day incubation period; Day 0 (black), Day 4 (blue), Day 8 (red), and Day 12 (green). 
Figure 2.6 shows the dUVRR spectra of the $A \beta$ protein treated with piceatannol on Day 0 when it was prepared and almost two weeks later on Day 12. The spectra were deconvoluted with each peak representing an amide or aromatic mode in the protein. Over time, there is no observable change in the spectra, however key differences are highlighted through difference spectra shown later.

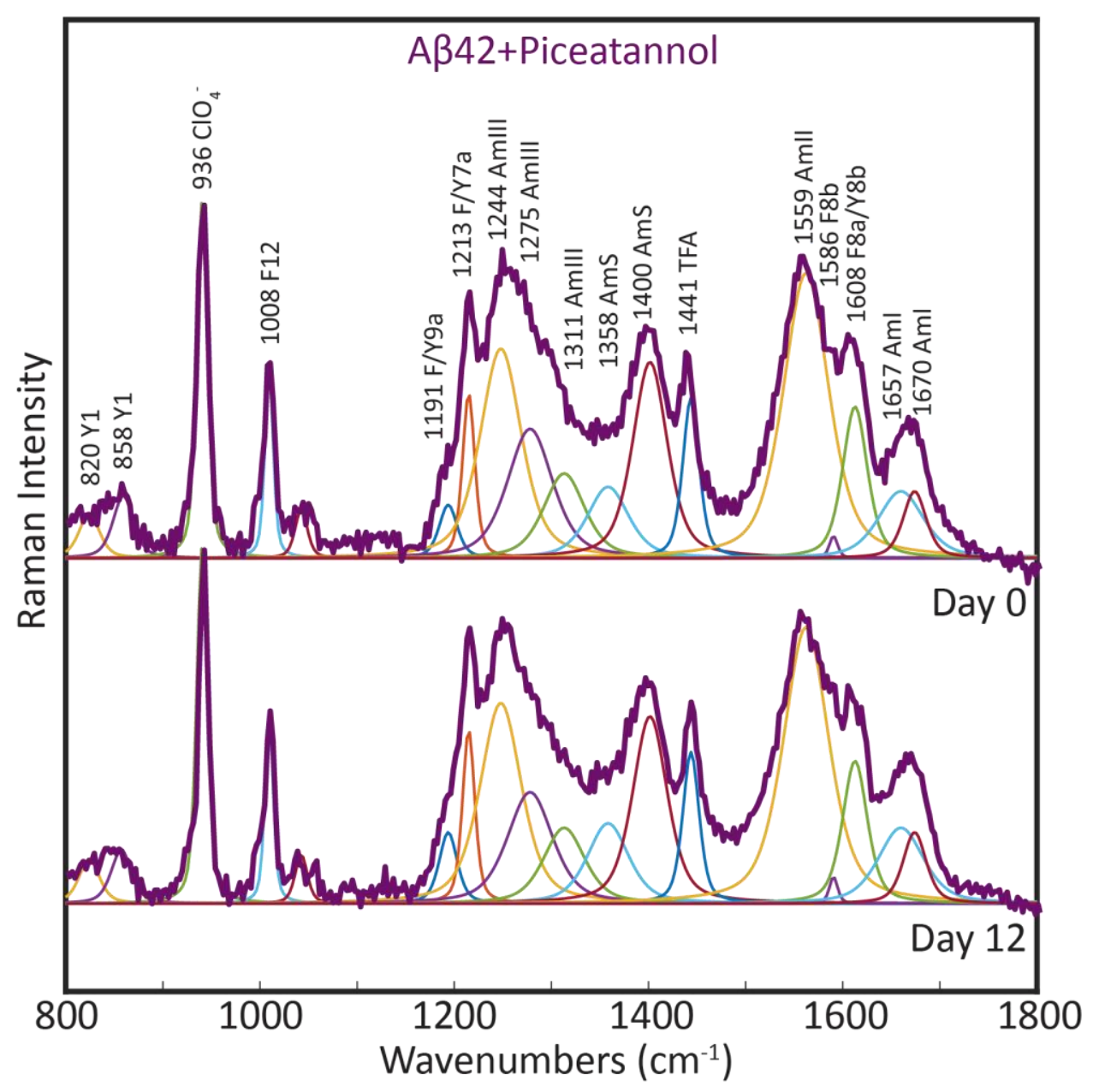

Figure 2.6: Deconvoluted dUVRR spectra of AB42 treated with piceatannol on Days 0 and 12 (purple). Deconvolution of the individual amide and aromatic modes was performed and those peaks are shown under each spectrum. 
Figure 2.7 shows the overlaid spectra of untreated $A \beta$ and $A \beta$ treated with piceatannol on Days 0 and 12. Differences are seen in amide III at $1244 \mathrm{~cm}^{-1}$, amide $\mathrm{S}$ at $1400 \mathrm{~cm}^{-1}$, and amide II at $1559 \mathrm{~cm}^{-1}$ which are stronger in the treated versus untreated spectrum on Day 12. Differences in the $\mathrm{Y}$ and $\mathrm{F}$ modes are seen predominantly in the $\mathrm{Y}$ modes at 820 and $858 \mathrm{~cm}^{-1}$, and the $\mathrm{Y} / \mathrm{F}$ modes at 1191,1586 , and $1608 \mathrm{~cm}^{-1}$.

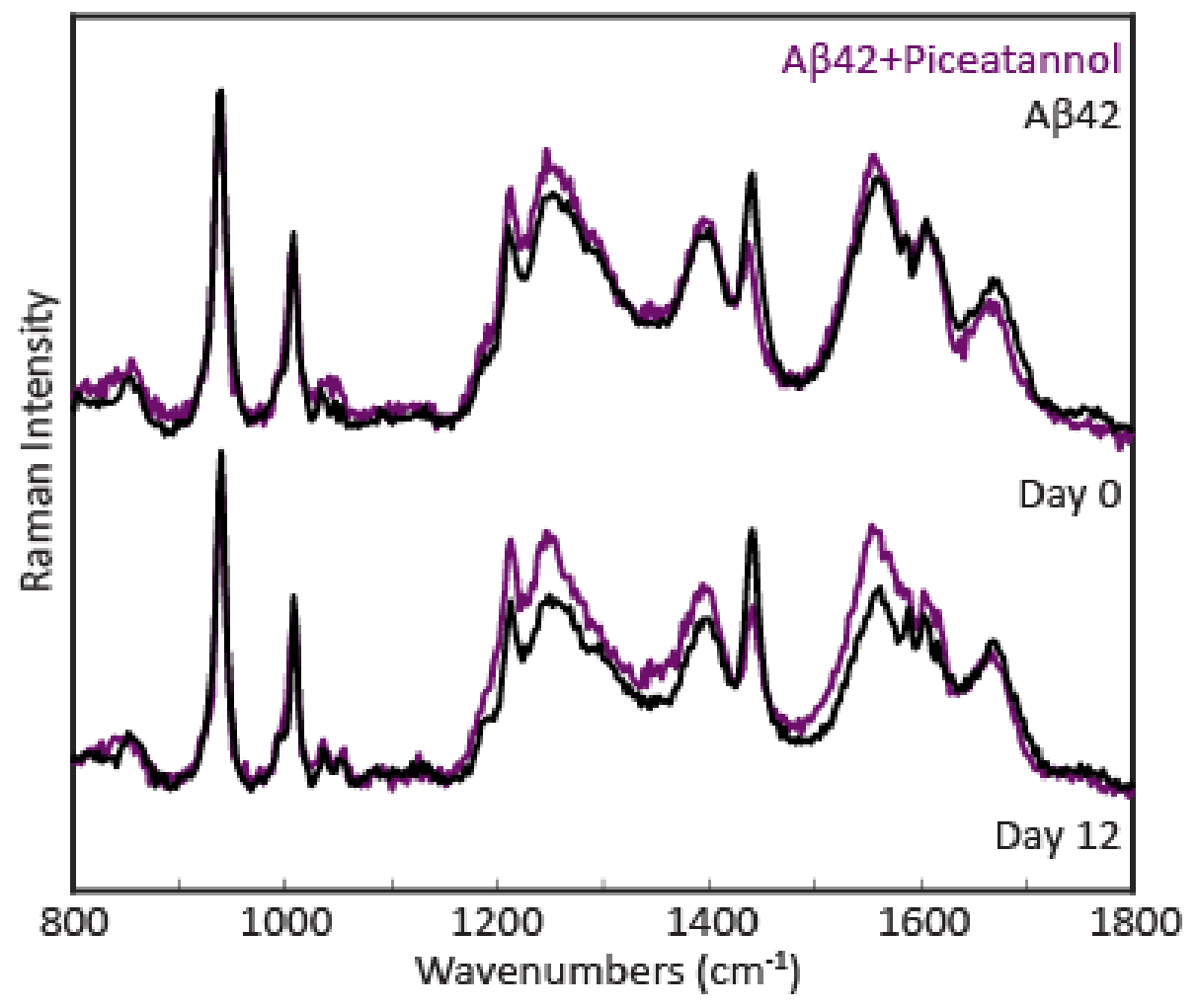

Figure 2.7: DUVRR spectra of AB42 (black) overlaid with AB42 treated with piceatannol (purple) on Days 0 and 12. 
Figure 2.8 shows the difference between the untreated $A \beta$ protein spectrum and the piceatannol-treated $A \beta$ spectrum on Days 0 and 12 . The difference spectra on Days 0 and 12 highlight that the amide modes are stronger in the dUVRR spectra of the treated samples. Modes associated with tyrosine at 858, 1191, 1213, and around 1608 $\mathrm{cm}^{-1}$ are increased in intensity in the dUVRR spectra of the treated samples. Interestingly, modes associated with phenylalanine at 1008,1586 , and $1608 \mathrm{~cm}^{-1}$ are decreased in intensity.

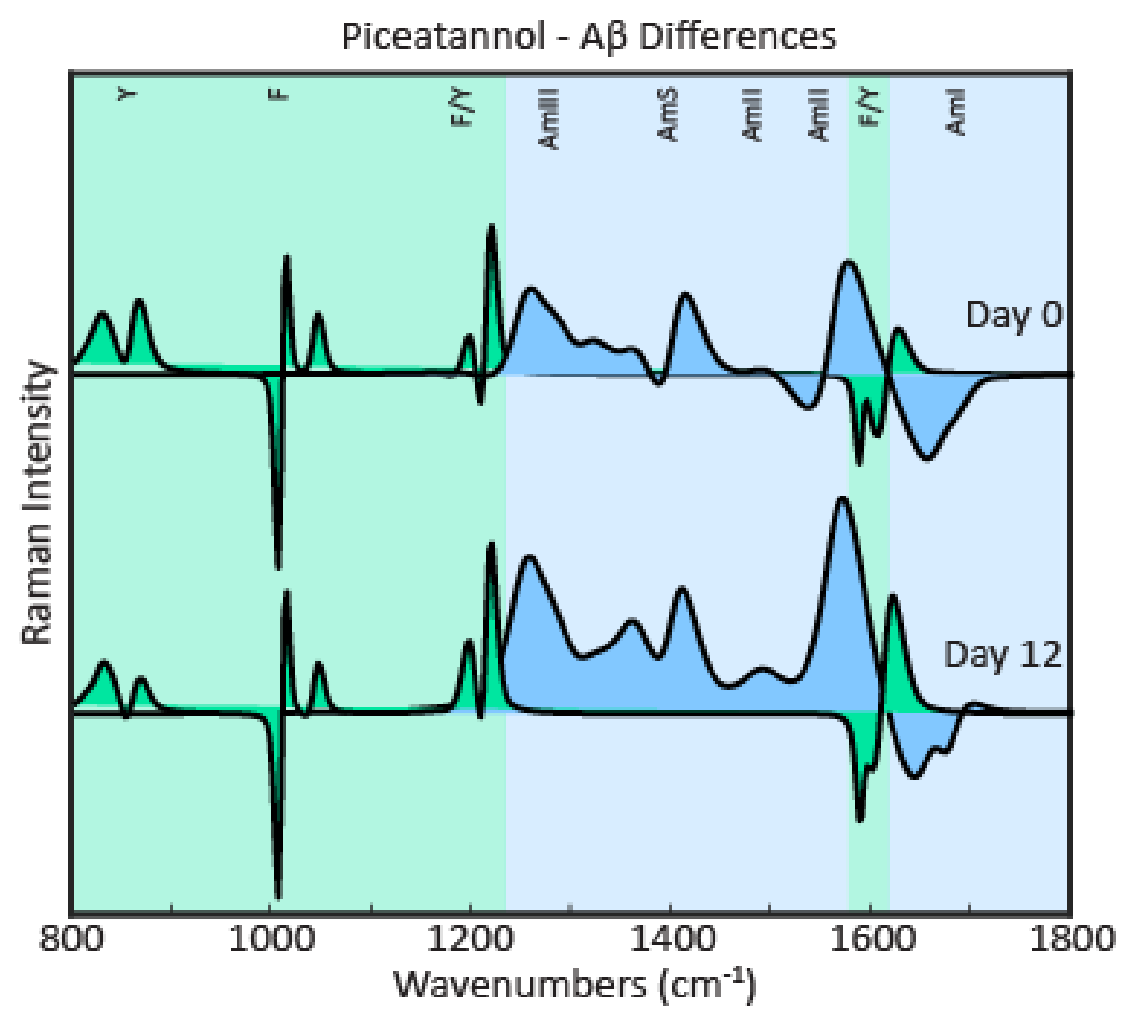

Figure 2.8: DUVRR difference spectra of the deconvoluted peaks of untreated AB42 and AB42 treated with piceatannol on Day 0 and Day 12. The amide modes are shown in blue and the aromatic modes are shown in green. 


\subsubsection{Resveratrol}

Figure 2.9 shows the $C D$ spectra of $A \beta$ treated with resveratrol during the 12 -day incubation period. Initially on Day 0 , the CD spectrum indicates that the protein is disordered in structure as indicated by the strong minimum at $197 \mathrm{~nm}$. The intensity of the minimum at $197 \mathrm{~nm}$ decreases with increasing incubation time, suggesting that the protein is changing structure. A small minimum begins to form at $217 \mathrm{~nm}$ by day 4, which is indicative of $\beta$-sheet structure. Addition of resveratrol does not prevent the $A \beta$ from forming $\beta$-sheet structure during incubation. Resveratrol may slow the formation of $\beta$-sheet structure, but ultimately it does not prevent aggregation.

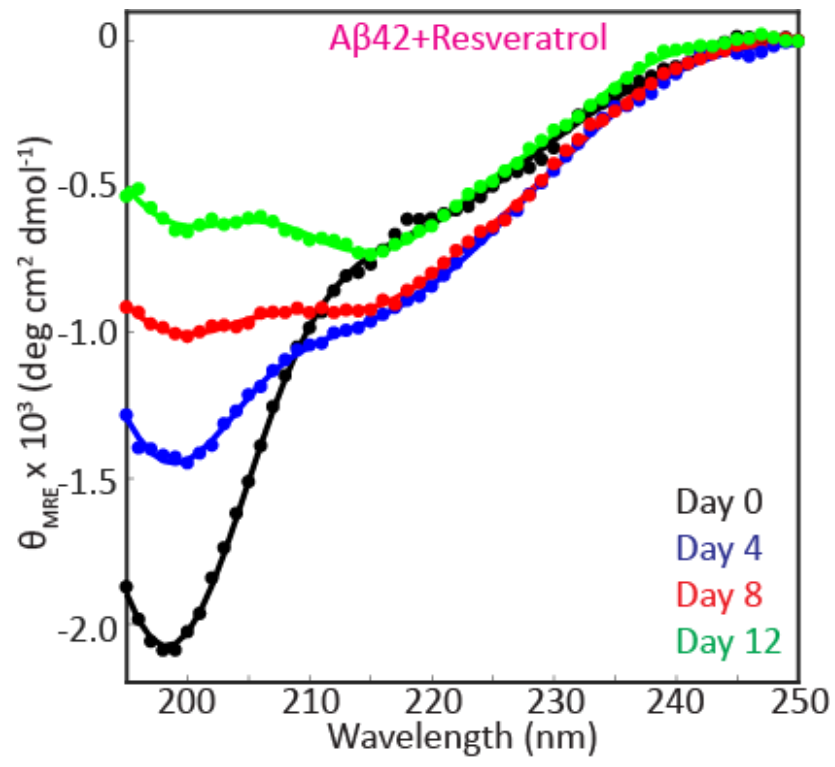

Figure 2.9: CD spectra of $A B 42$ treated with resveratrol during a 12-day incubation period; Day 0 (black), Day 4 (blue), Day 8 (red), and Day 12 (green). 
Figure 2.10 shows the dUVRR spectra of the $A \beta$ protein treated with resveratrol on Days 0 and 12. The spectra were deconvoluted in to a series of peaks that represent the amide and aromatic modes of the protein. Over the 12-day incubation period, there is no pronounced change in the spectra, however key differences are highlighted in the difference spectra shown later.

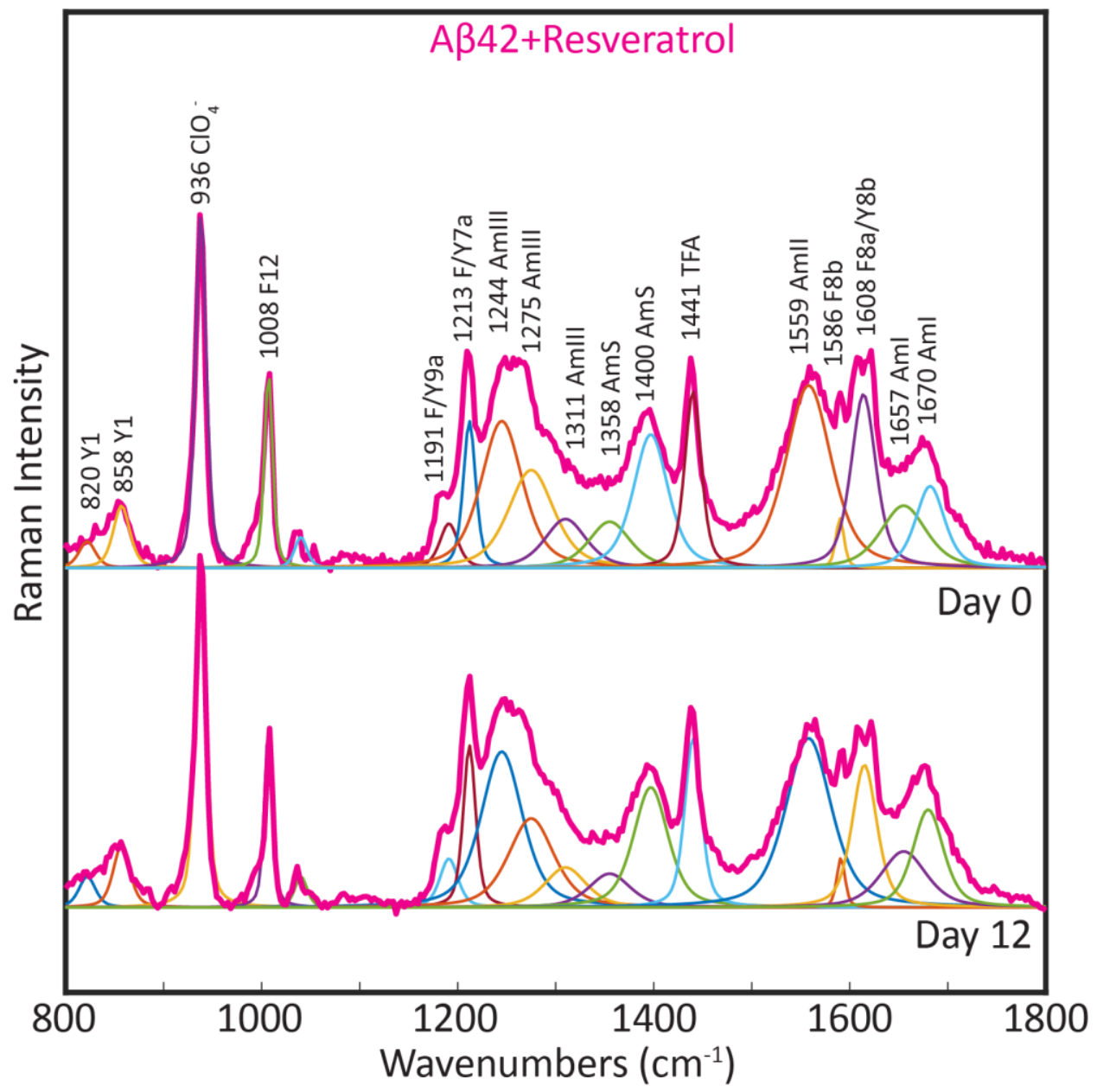

Figure 2.10: Deconvoluted dUVRR spectra of AB42 treated with resveratrol on Days 0 and 12 (pink). Deconvolution of the individual amide and aromatic modes was performed and those peaks are shown under each spectra. 
Figure 2.11 shows the overlaid dUVRR spectra of untreated $A \beta$ and $A \beta$ treated with resveratrol on Days 0 and 12 . There are more differences between the Day 0 spectra than the Day 12 spectra. The CD spectra show that initially the protein is disordered, but it eventually forms $\beta$-sheet structure. The similarity of the Day 12 spectra suggest that any differences in structure initially between the two treatments are likely gone after 12 days of incubation.

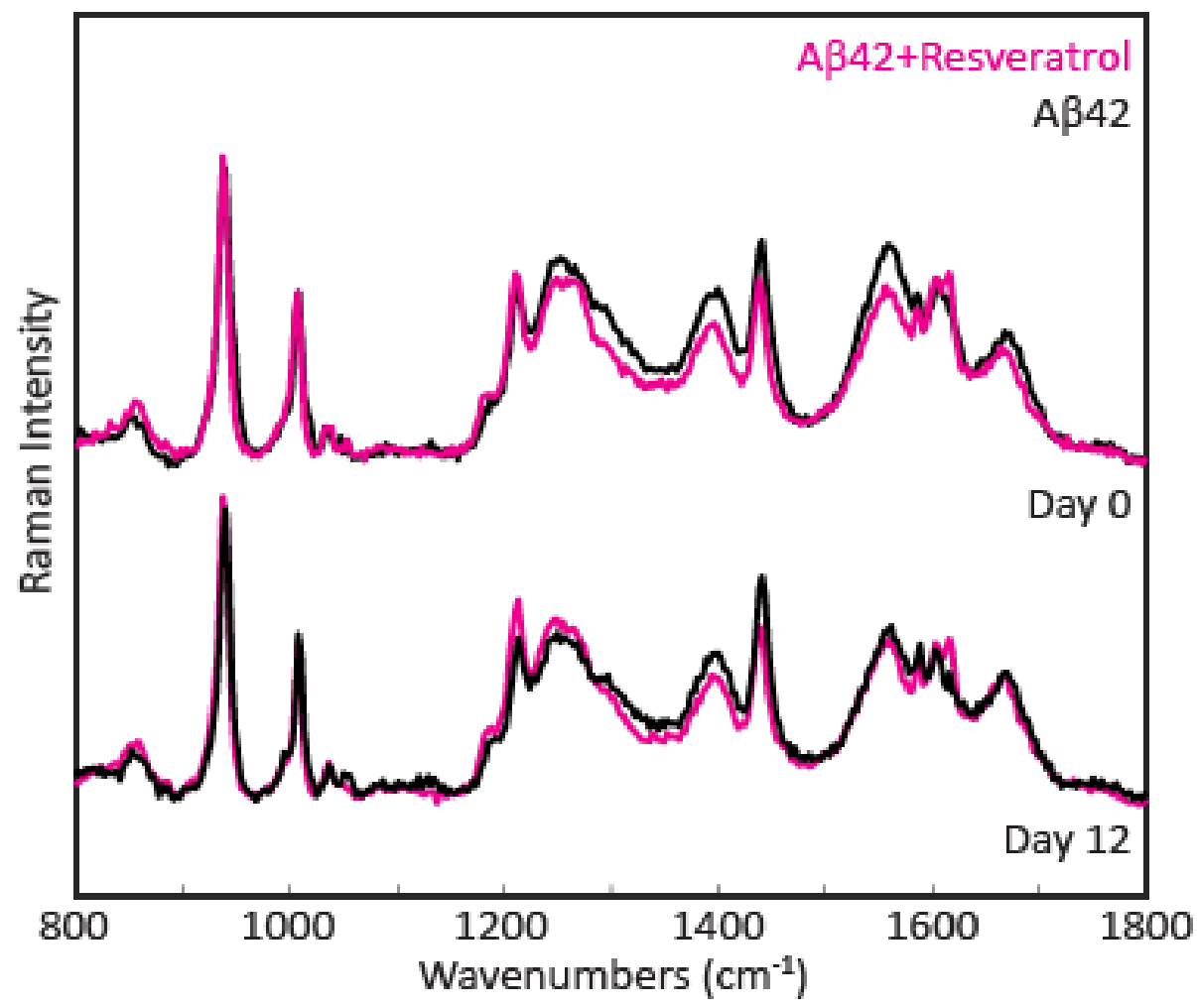

Figure 2.11: DUVRR spectra of AB42 (black) overlaid with AB42 treated with resveratrol (pink) on Days 0 and 12. 
Figure 2.12 shows the difference spectra of the untreated $A \beta$ protein and resveratrol-treated $A \beta$ on Days 0 and 12 . Initially there is a large difference in the intensity of the amide modes but this difference is reduced over time, likely due to the fact resveratrol does not prevent formation of $\beta$-sheet structure. The aromatic modes associated with tyrosine are stronger in the dUVRR spectra of $A \beta$ treated with resveratrol but the phenylalanine modes at $1008 \mathrm{~cm}^{-1}$ is not.

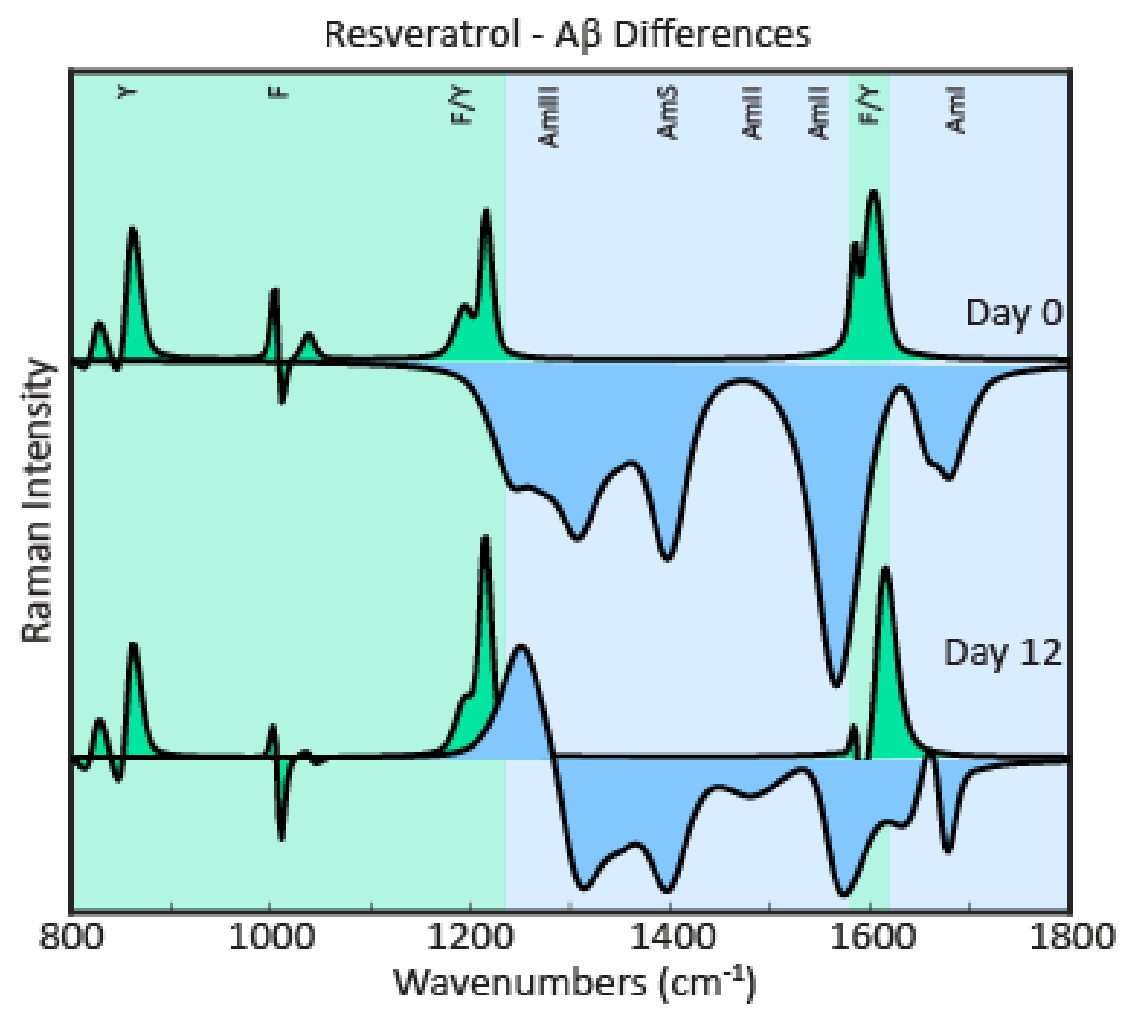

Figure 2.12: DUVRR difference spectra of the deconvoluted peaks of untreated $A B 42$ and $A B 42$ treated with resveratrol the untreated $A B$ on Day 0 and Day 12. The amide modes are shown in blue and the aromatic modes are shown in green. 


\subsubsection{Diethylstilbestrol}

Figure 2.13 shows the $C D$ spectra of diethylstilbestrol-treated $A \beta$ during the incubation period. Initially on Day 0 , the CD spectrum indicates that the protein is largely disordered as indicated by the minimum at $197 \mathrm{~nm}$. However, the CD spectrum quickly loses the negative feature at $197 \mathrm{~nm}$ and develops the characteristic $\beta$-sheet associated minimum at $217 \mathrm{~nm}$. After Day 4, the CD spectrum remains stable indicating the structure of the protein remains unchanged for the rest of the incubation period.

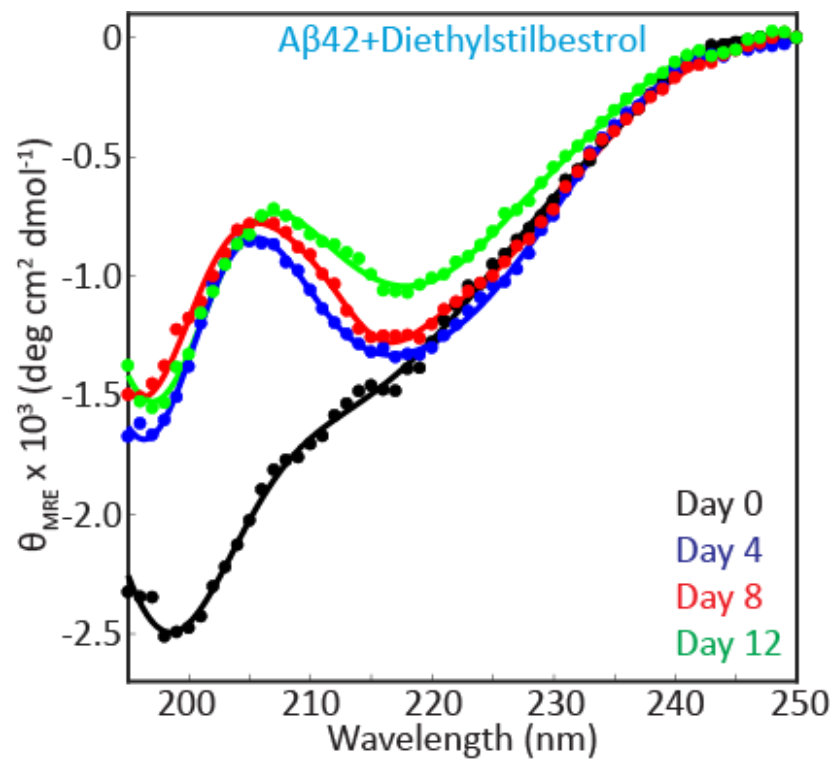

Figure 2.13: CD spectra of $A B 42$ treated with diethylstilbestrol during a 12-day incubation period; Day 0 (black), Day 4 (blue), Day 8 (red), and Day 12 (green). 
Figure 2.14 shows the dUVRR spectrum of the $A \beta$ protein treated with

diethylstilbestrol on Days 0 and 12 . With respect to the internal intensity standard perchlorate, the entire spectrum decreases in intensity. A decrease in overall intensity may be associated with formation of insoluble $\beta$-sheet oligomers.

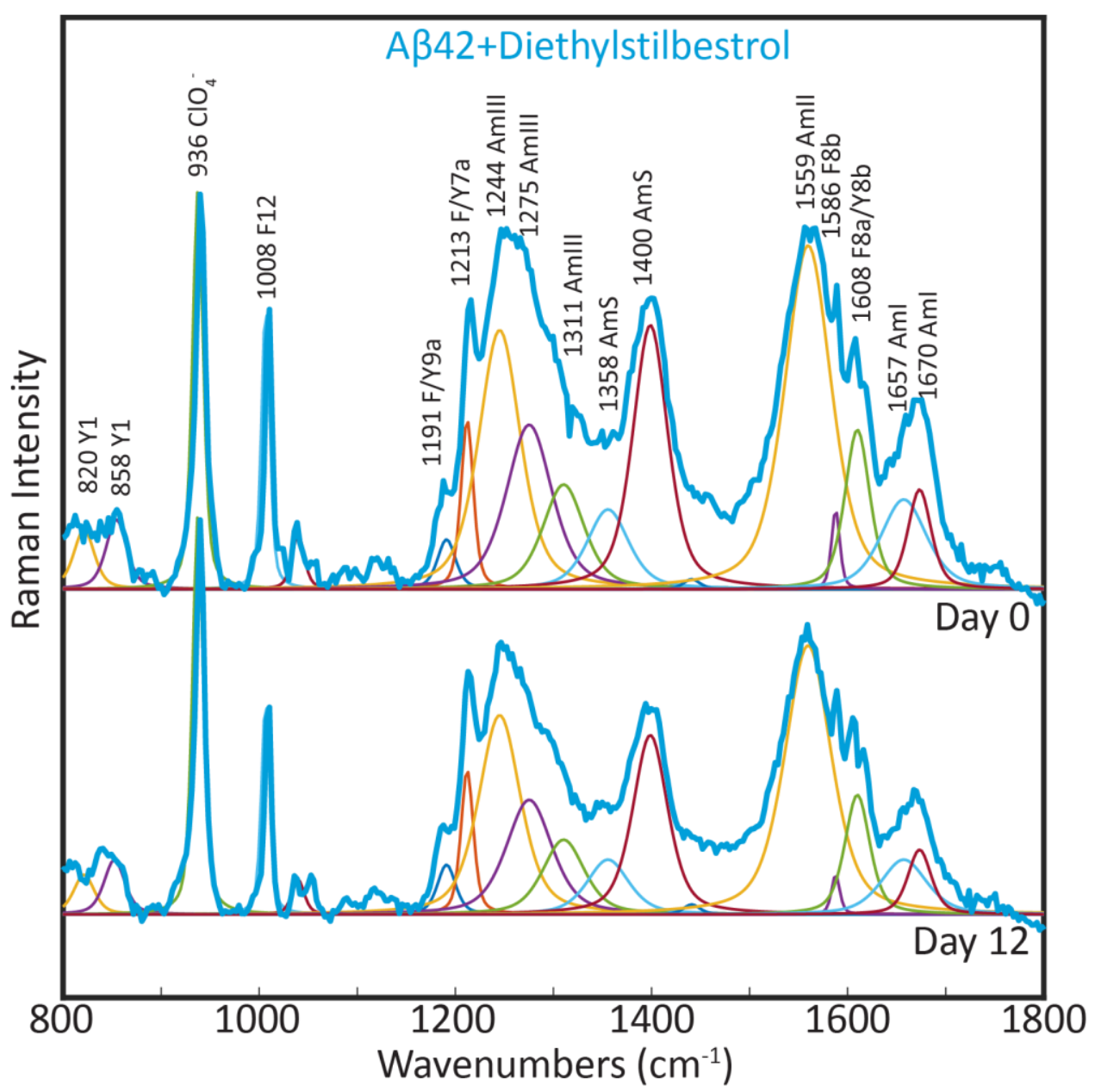

Figure 2.14: Deconvoluted dUVRR spectra of AB42 treated with diethylstilbestrol on Day 0 and Day 12 (blue). Deconvolution of the individual amide and aromatic modes was performed and those peaks are shown under each spectra. 
Figure 2.15 shows the overlaid spectra of untreated $A \beta 42$ and $A \beta 42$ treated with diethylstilbestrol on Days 0 and 12. The most pronounced differences between the untreated and diethylstilbestrol-treated $A \beta$ spectra are in the amide III and amide I regions. Differences in the aromatic modes of $Y$ at $820 \mathrm{~cm}^{-1}$ and $F$ at 1586 and $1608 \mathrm{~cm}^{-1}$ were also apparent. The spectrum of untreated $A \beta$ also has a TFA peak that is not present in the diethylstilbestrol-treated sample spectrum, as the counter ion for the protein was changed to hydrochloride which does not have a feature in dUVRR spectra.

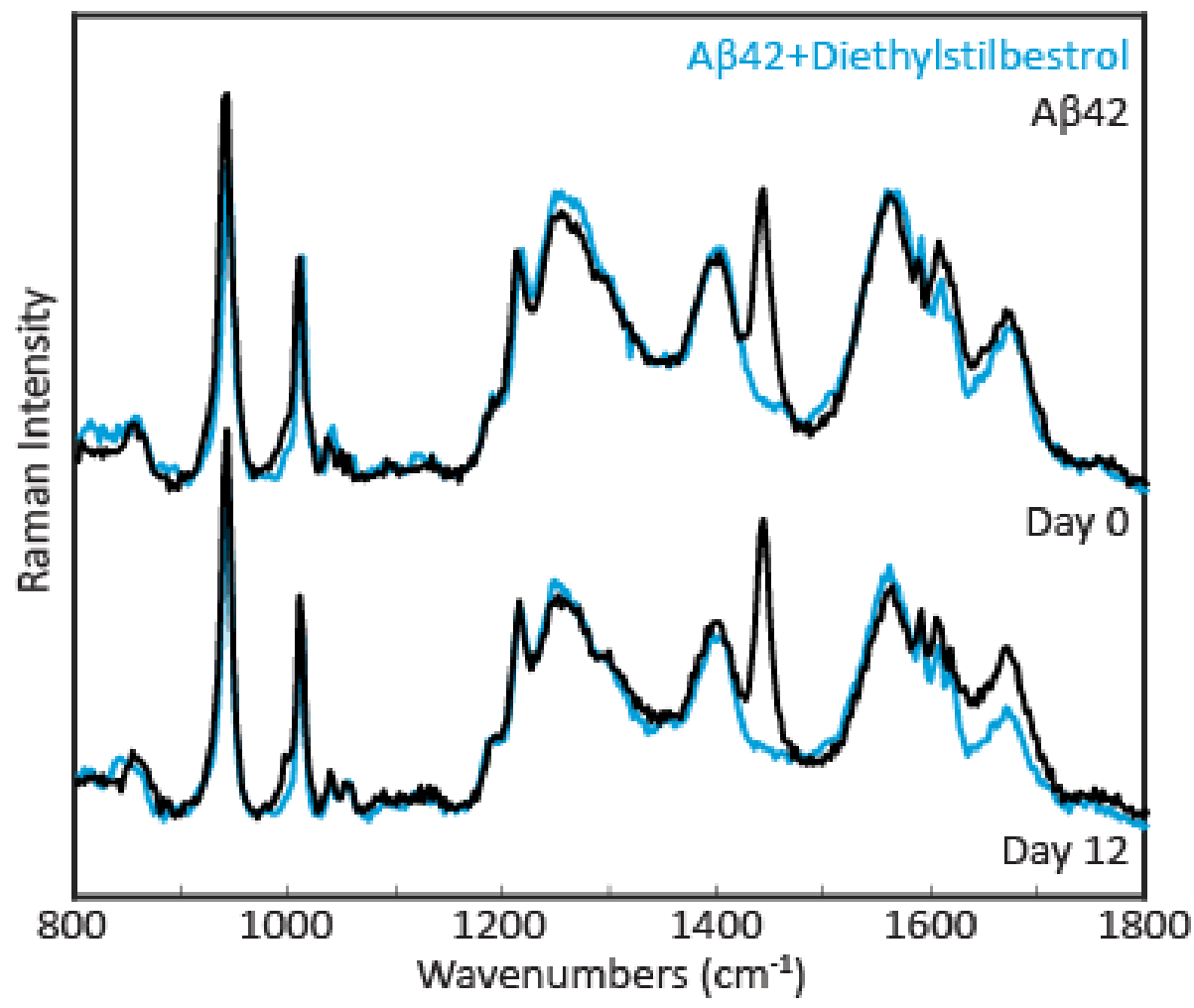

Figure 2.15: DUVRR spectra of AB42 (black) overlaid with AB42 treated with diethylstilbestrol (blue) on Days 0 and 12. 
Figure 2.16 shows the difference spectra for the untreated $A \beta$ protein and diethylstilbestrol-treated $A \beta$ protein on Days 0 and 12 . The amide II band at $1244 \mathrm{~cm}^{-1}$ is more intense suggesting the treated sample may have more $\beta$-sheet structure than the untreated sample initially. Interestingly, the amide I band is less intense. The aromatic bands are also decreased in intensity.

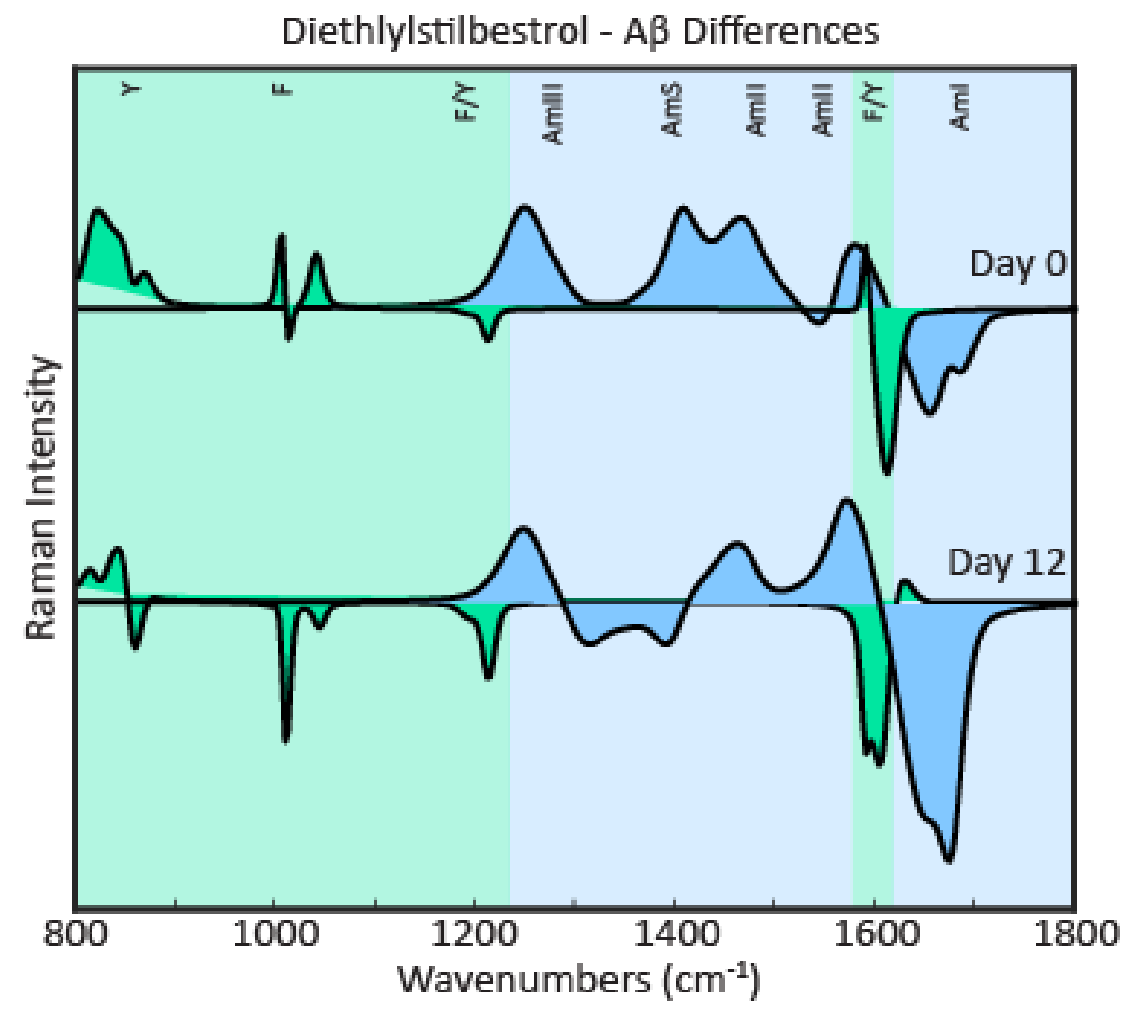

Figure 2.16: DUVRR difference spectra of the deconvoluted peaks of untreated $A B 42$ and $A B 42$ treated with diethylstilbestrol on Day 0 and Day 12. The amide modes are shown in blue and the aromatic modes are shown in green.

\subsection{Discussion}

The number of hydroxyl substituents on the rings of the stilbenoids used to treat $A \beta$ samples appears to be positively correlated with the polyphenol's ability to prevent $\beta$-sheet formation. Specifically, more substituents on the rings of the stilbenoid traps the peptide in a disordered state as confirmed by CD. On Day 0, each of the polyphenol- 
treated samples are disordered as evidenced by the strong negative feature at $197 \mathrm{~nm}$ in their CD spectra. By Day 4, the negative intensity at $197 \mathrm{~nm}$ is reduced in CD spectra of all samples, but to differing degrees. The piceatannol-treated sample remained largely disordered, while the sample treated with resveratrol has an observable amount of $\beta$-sheet structure. Samples treated with diethylstilbestrol lost their disordered structure completely by Day 4 and formed $\beta$-sheet structure.

As the number of hydroxyl substituents decrease, there is a trend in the effect on the aromatic modes as seen in Figure 2.17 . The phenylalanine mode at $1008 \mathrm{~cm}^{-1}$ was most intense in the dUVRR spectrum of $A \beta$ treated with piceatannol, which has the greatest number of hydroxyl substituents. As the number of hydroxyl substituents decreases, the intensity of the $1008 \mathrm{~cm}^{-1}$ band decreased. The overlapped ring stretching $\mathrm{Y} / \mathrm{F}$ mode at $1213 \mathrm{~cm}^{-1}$ was increased in dUVRR spectra of samples treated with piceatannol or resveratrol but decreased in samples treated with diethylstilbestrol.

As the number of substituents on the rings of the stilbenoids decrease, aromatic modes mainly decrease in intensity. As the number of substituents increase, there appears to be less interaction between the stilbenoid and the aromatic residues of the protein. Decreasing the polarity of the chain linker may also yield a stronger interaction between the polyphenol and the aromatic residues of the protein. This is seen in Figure 2.17 where the aromatic modes are most perturbed in the dUVRR spectra of samples treated with diethylstilbestrol. Decreasing the number of substituents, reduces the polarity of the polyphenol. This may be allowing for more pi-stacking interactions with 
the protein yielding greater changes in the aromatic bands than when more hydroxyl groups are present on the ring.

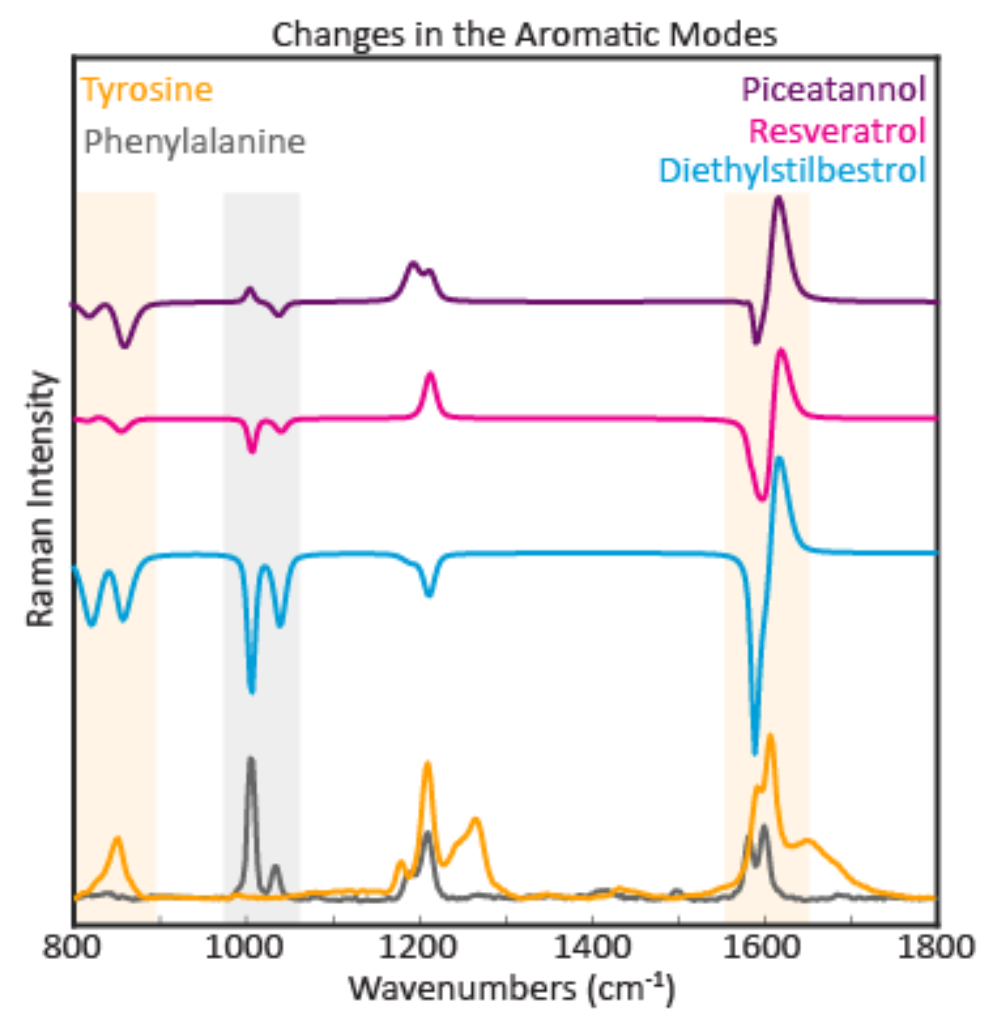

Figure 2.17: Changes over the 12-day incubation period in the individual aromatic modes of the dUVRR spectra. Diethylstilbestrol (blue), resveratrol (pink), and piceatannol (purple) are shown. 


\subsection{References}

1. Lakey-Beitia, J.; Berrocal, R.; Rao, K. S.; Durant, A. A., Polyphenols as therapeutic molecules in Alzheimer's disease through modulating amyloid pathways. Mol Neurobiol 2015, 51 (2), 466-479.

2. Porat, Y.; Abramowitz, A.; Gazit, E., Inhibition of amyloid fibril formation by polyphenols: structural similarity and aromatic interactions as a common inhibition mechanism. Chem Biol Drug Des 2006, 67 (1), 27-37.

3. Gauci, A. J. C., M.; Giese, A.; Scerri, C.; Vassallo, V. , Identification of polyphenolic compounds and black tea extract as potent inhibitors of lipid membrane destabilization by A beta(42) aggregates - ResearchGate. J of Alz Dis 2011, 24 (4), 767-779.

4. Ono, K.; Yoshiike, Y.; Takashima, A.; Hasegawa, K.; Naiki, H.; Yamada, M., Potent antiamyloidogenic and fibril-destabilizing effects of polyphenols in vitro: implications for the prevention and therapeutics of Alzheimer's disease. J Neurochem 2003, 87 (1), 172-181.

5. Lai, N. H.; Herent, M. F.; Leclercq, J. Q.; Nguyen, B. T.; Rogez, H.; Larondelle, Y.; Andre, C. Piceatannol, a potent bioactive stilbene, as major phenolic component in Rhodomyrtus tomentosa. Food Chem 2013, 138 (2-3), 1421-1430.

6. Burns, J.; Yokota, T. ; Ashihara, H.; Lean M. E.; Crozier, A. Plant foods and herbal sources of resveratrol. J Agric Food Chem 2002, 50 (11), 3337-3340.

7. Adam, E.; Decker, D. G.; Herbst, A. L.; Noller, K. L.; Tilley, B. C.; Townsend, D. E. Exposure in utero to diethylstilbestrol and related synthetic hormones: Association with vaginal and cervical cancers and other abnormalities. JAMA 1976, 236 (10), 1107-1109.

8. Wang, M.; JiJi, R.D. Spectroscopic detection of $\beta$-sheet structure in nascent $A \beta$ oligomers. J Biophotonics 2011, 4, 637-644.

9. Simpson, J. V.; Balakrishnan, G.; JiJi, R. D. MCR-ALS analysis of two-way UV resonance Raman spectra to resolve discrete protein secondary structural motifs. Analyst 2009, 134, 138-147.

10. Wang, M.; JiJi, R. D. Resolution of localized small molecule-A $\beta$ interactions by deep-ultraviolet resonance Raman spectroscopy. Biophys Chem 2011, 158, 96-103. 


\section{Chapter III: Increasing Chain Linker Polarity Increases $\beta$-sheet Formation}

\subsection{Abstract}

Diaryl polyphenolic compounds have common structural components consisting of two phenolic rings with hydroxyl group substituents and a chain linker between the rings. The chain between the two phenol rings can vary significantly both in length and atom makeup, affecting the potency of the polyphenol. Flavonoid compounds do not contain a chain linker and understanding how the chain linker can affect the activity of non-flavonoid compounds is imperative. The aromatic rings of polyphenols are important for their binding to proteins and effects on protein aggregation. The type of linker between the aromatic rings of diaryl polyphenols is also important because it could affect $A \beta$ binding and aggregation. The diaryl polyphenols nordihydroguaiaretic acid, curcumin, and rosmarinic acid have increasing polarity, respectively, in the chain linker between the phenolic rings. Each of these polyphenols has four ring substituents and 4 - 6 atoms in their chain linker. It this study, it was found that with increasing polarity of the linker, the polyphenol-treated $A \beta$ sample had a greater tendency to form a $\beta$-structure. Thus, in order to maintain $A \beta$ in a disordered structure (non-fibrillar), the linker between the phenol rings should be of low polarity. A polar linker leads to a reduction in disordered content and an increase in $\beta$-sheet structure in the protein. 


\subsection{Introduction}

The chain linker between the two phenol rings can vary significantly both in length and atom makeup, and has an important impact on how the polyphenols will metabolize and behave. ${ }^{[1-2]}$ It is suggested that the optimum chain length for inhibiting amyloid formation between the rings should be 2-6 atoms long. ${ }^{[3]}$ Studies have shown that diaryl polyphenols such as curcumin, are better than flavonoids at inhibiting $A \beta^{\prime} s$ amyloidogenic activity, possibly due to the type of chain linker between the aromatic rings. ${ }^{[1-2]}$

The aromatic rings are important for polyphenol binding to proteins and for inhibiting protein aggregation. The aromatic rings of polyphenols participate in hydrophobic interactions with proteins that can interrupt endogenous hydrophobic interactions within the protein. The endogenous interactions promote aggregation of $A \beta$ by maintaining its stability and orienting the protein for self-assembly of organized structures. ${ }^{[3,4-9]}$ The rings of polyphenols have the necessary planarity to bind to the protein in the hydrophobic groove, interrupt the $\pi$ stacking, and preventing aggregation by intercalating within the $\beta$-sheets. ${ }^{[1,4,10-11]}$ The chain linker polarity will affect the polyphenol's overall polarity and its ability to disrupt hydrophobic interactions necessary for protein aggregation.

The polyphenols nordihydroguaiaretic acid, curcumin, and rosmarinic acid have increasing polarity, respectively, within the chain linker region between their phenolic rings. Each of these polyphenols have four ring substituents and $4-6$ atoms in their chain linker. 
Nordihydroguaiaretic acid is a plant based polyphenol found in the leaves of a desert shrub, the creosote bush and is valued for its anti-inflammatory effects. ${ }^{[12]}$ Nordihydroguaiaretic acid, shown in Figure 3.1, is the least polar of the polyphenols evaluated in this study. It has four atoms in the chain linker between the rings with two methyl substituents off the second and third carbons. It has a total of four hydroxyl groups on the rings as well.

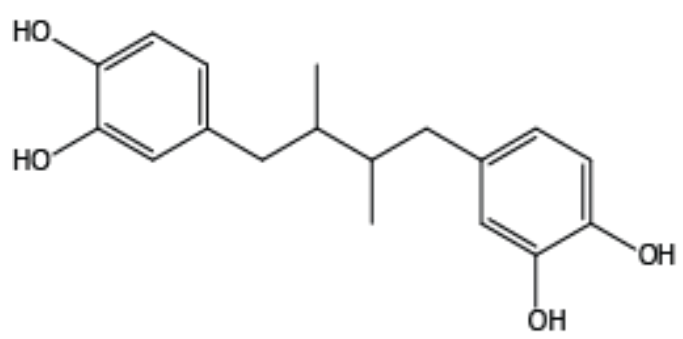

Figure 3.1: Structure of nordihydroguaiaretic acid.

Curcumin is the principal curcuminoid found in turmeric and has not only been used as a spice, but for its anticancer and anti-inflammatory effects as well. ${ }^{[13]}$ Curcumin, shown in Figure 3.2, is more polar than nordihydroguaiaretic acid, but less polar than rosmarinic acid. It has five atoms in the chain linker between the rings with one hydroxyl substituent off the second carbon and a ketone off the fourth carbon. It has a total of two hydroxyl groups on the rings as well as two ether groups.

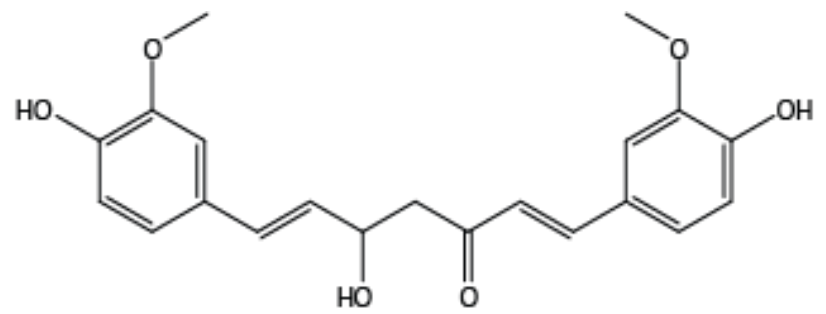

Figure 3.2: Structure of curcumin.

Rosmarinic acid is commonly found in many spices such as sage, mint, thyme, basil, and rosemary where it developed its name. ${ }^{[14]}$ Rosmarinic acid, shown in Figure 
3.3, is the most polar of the three polyphenols evaluated. It has six atoms in the chain linker between the rings with an ester and carboxylic acid on the third and fourth carbons, respectively.

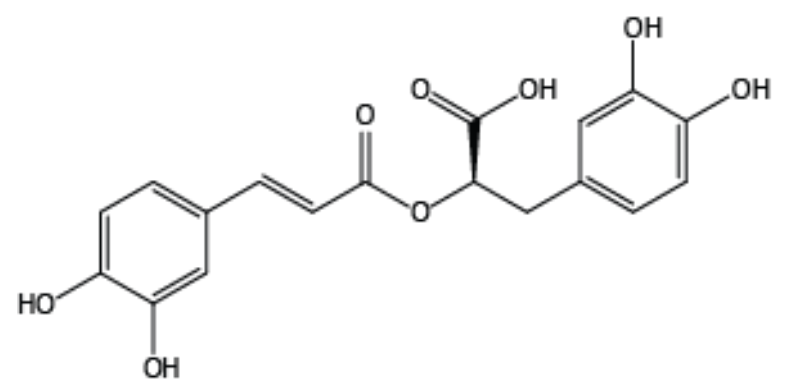

Figure 3.3: Structure of rosmarinic acid.

This study aims to discover how the polarity of the chain linker of polyphenols can affect $A \beta$ aggregation and $\beta$-sheet formation over time.

\subsection{Materials and Methods}

\subsubsection{Materials}

$A \beta$ (1-42) (>95\% pure) was purchased from Atlantic Peptides (Scarborough, ME). Ethanol and sodium perchlorate were purchased from Sigma-Aldrich (St. Louis, MO) and used without further purification. Sodium phosphate monobasic, sodium phosphate dibasic, sodium chloride, and cyclohexane were purchased from Fisher Scientific (Pittsburgh, PA). Rosmarinic acid and nordihydroguaiaretic acid were purchased from Sigma-Aldrich (St. Louis, MO) and curcumin was purchased from Acros Organics (Pittsburgh, PA).

\subsubsection{Spectroscopic Measurements and Data Analysis}

The amyloid- $\beta$ (1-42) protein was prepared and characterized as described in Chapter 2, section 2.3.2. Circular dichroism (CD) and deep-ultraviolet resonance Raman 
(dUVRR) were collected, processed, and analyzed as described in Chapter 2, sections

\subsection{3-2.3.5.}

\subsection{Results}

\subsubsection{AB Untreated}

Figure 3.4 shows the $C D$ spectra of $A \beta$ during the 12-day incubation period.

Initially on Day 0, the protein is a disordered in structure, as indicated by the minimum at $197 \mathrm{~nm}$. Over time, this feature decreases in intensity and another minimum forms at $217 \mathrm{~nm}$ indicative of $\beta$-sheet formation.

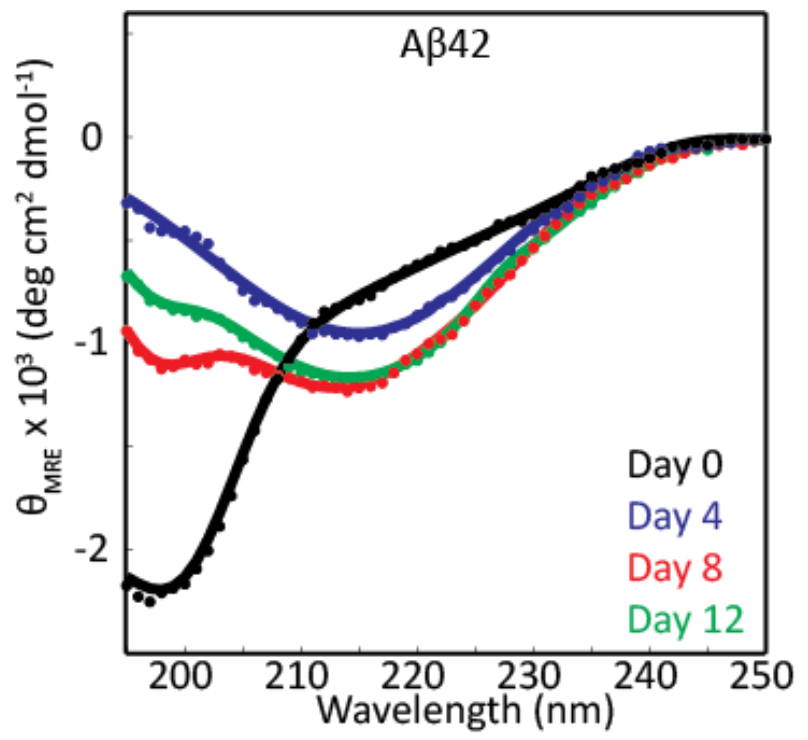

Figure 3.4: CD spectra of AB42 during a 12-day incubation period; Day 0 (black), Day 4 (blue), Day 8 (red), and Day 12 (green). 


\subsubsection{Nordihydroguaiaretic Acid}

Figure 3.5 shows the $C D$ spectra of nordihydroguaiaretic acid-treated $A \beta$ during the incubation period. Similar to the untreated $A \beta$ sample, on Day 0 , the treated protein is a disordered in structure, as indicated by the minimum at $197 \mathrm{~nm}$. There is a slight loss of intensity at $197 \mathrm{~nm}$ on Day 4 but over time, the structure of the treated protein remains stable and disordered.

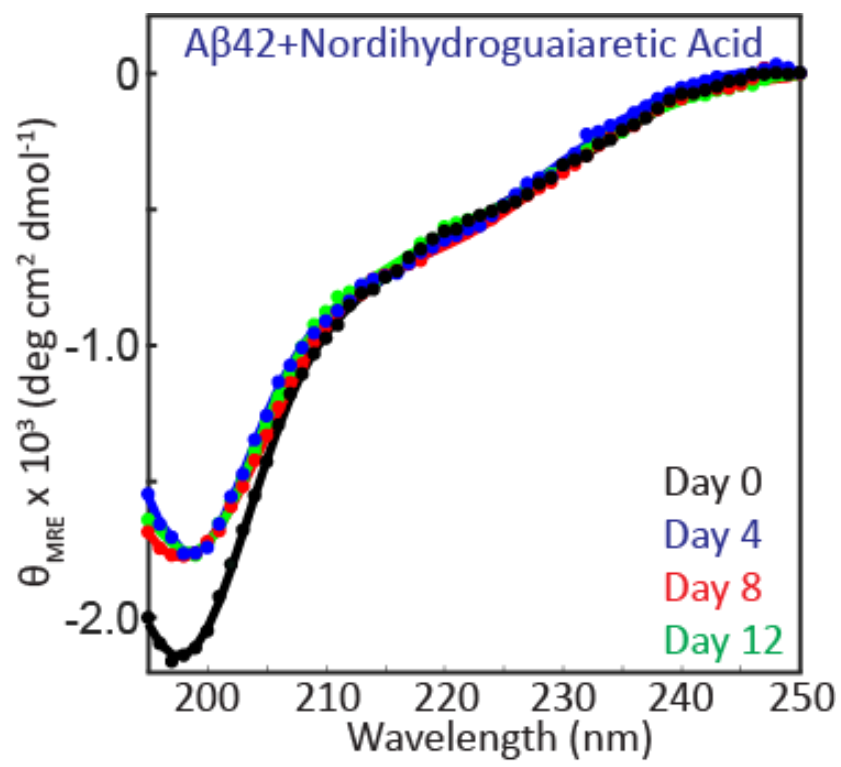

Figure 3.5: CD spectra of AB42 treated with nordihydroguaiaretic acid during a 12-day incubation period; Day 0 (black), Day 4 (blue), Day 8 (red), and Day 12 (green). 
Figure 3.6 shows the $A \beta$ protein treated with nordihydroguaiaretic acid on Days 0 and 12 . The most noteworthy difference is the peak at $1295 \mathrm{~cm}^{-1}$. This extra peak is attributed to the stretching of the hydroxyl groups on the rings of the polyphenol itself. Over time, there is no visible change between the Day 0 and Day 4 dUVRR spectra other than this peak from nordihydroguaiaretic acid. After 12 days, this peak disappears due to the oxidation of the polyphenol. The hydroxyl groups donate a hydrogen to the protein which could be crucial in preventing protein aggregation. Observing this peak on Day 0 and not on Day 12 confirms that the polyphenol is undergoing oxidation.

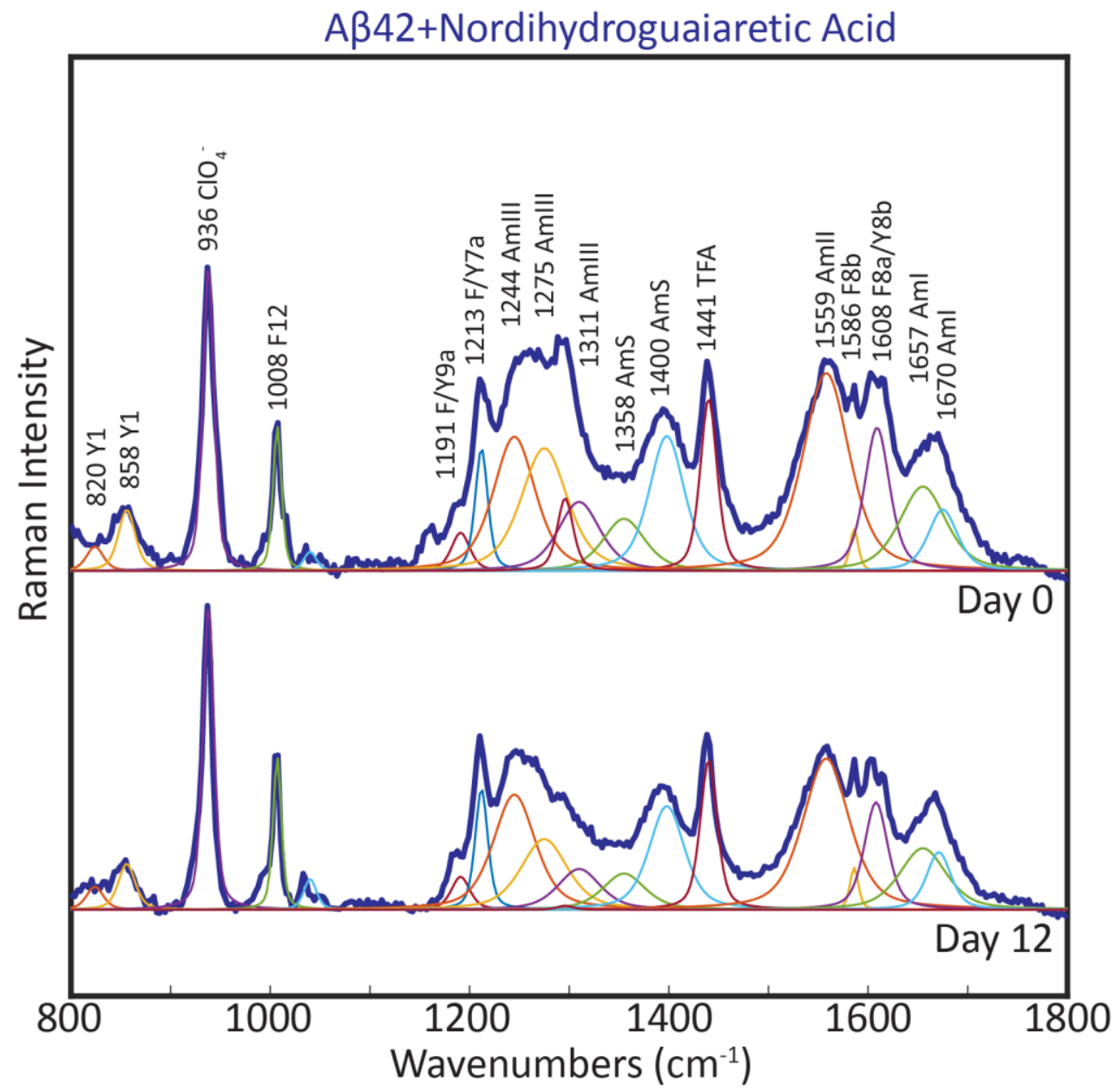

Figure 3.6: Deconvoluted dUVRR spectra of AB42 treated with nordihydroguaiaretic acid on Days 0 and 12 (blue). Deconvolution of the individual amide and aromatic modes was performed and those peaks are shown under each spectra. 
Figure 3.7 shows the overlaid spectra of untreated $A \beta$ and $A \beta$ treated with nordihydroguaiaretic acid on Days 0 and 12. The most pronounced difference between the untreated and treated $A \beta$ dUVRR spectra is the peak at $1295 \mathrm{~cm}^{-1}$ from the polyphenol. Other differences include a slight increase in the intensity of the $F / Y$ modes at 1586 and $1608 \mathrm{~cm}^{-1}$ as well as the $Y$ mode at $858 \mathrm{~cm}^{-1}$ for the treated sample.

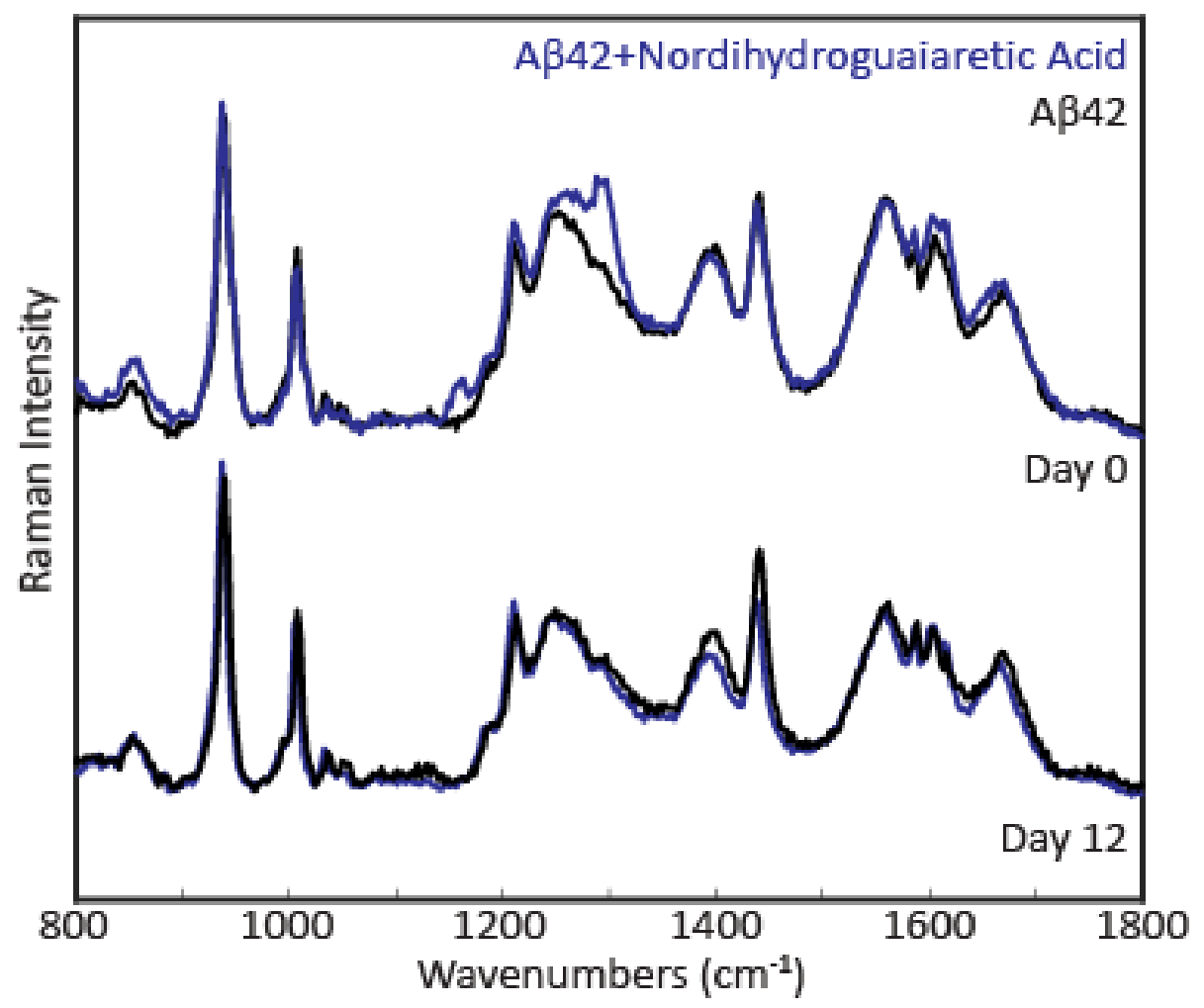

Figure 3.7: DUVRR spectra of AB42 (black) overlaid with AB42 treated with nordihydroguaiaretic acid (blue) on days 0 and 12 . 
Figure 3.8 shows the differences between the untreated $A \beta$ protein and the nordihydroguaiaretic acid-treated $A \beta$ dUVRR spectra on Days 0 and 12 . The largest difference between the Day 0 spectra is in the amide III region, which is primarily due to the loss of the $1295 \mathrm{~cm}^{-1}$ peak from the polyphenol. On Day 0, tyrosine associated modes appear to be stronger in the spectrum of the treated $A \beta$ sample, but the phenylalanine mode at $1008 \mathrm{~cm}^{-1}$ appears to have lower intensity. By Day 12 , the differences in the aromatic modes have diminished.

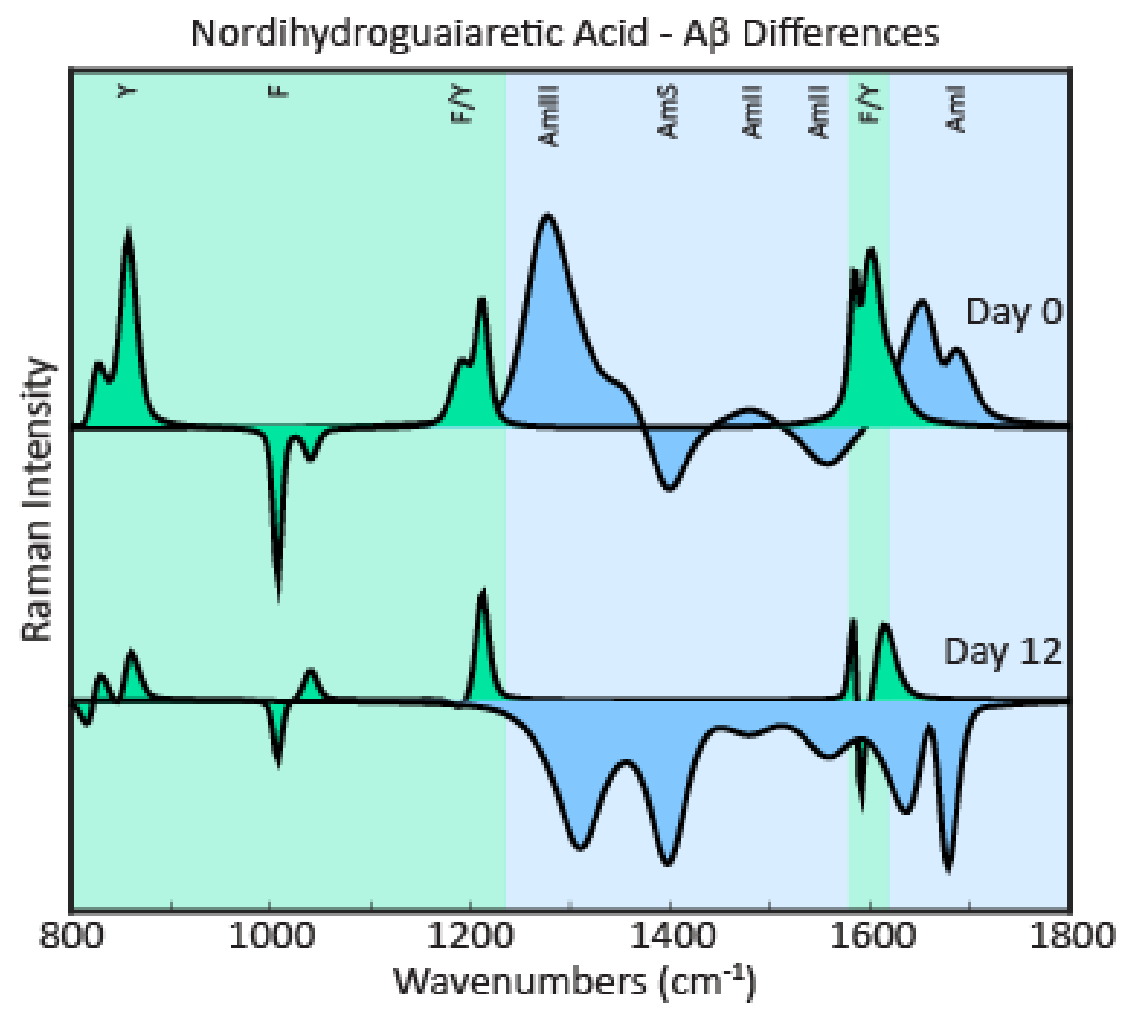

Figure 3.8: DUVRR difference spectra of the deconvoluted peaks of AB42 treated with nordihydroguaiaretic acid and the untreated $A B 42$ on Day 0 and Day 12. The amide modes are shown in blue and the aromatic modes are shown in green. 


\subsubsection{Curcumin}

Figure 3.9 shows the $C D$ spectra of curcumin-treated $A \beta$ during the incubation period. Initially on Day 0, the curcumin-treated protein is disordered in structure, as indicated by the minimum at $197 \mathrm{~nm}$. Although there is a slight loss of intensity at 197 $\mathrm{nm}$ after each time point, the treated protein remains in disordered state.

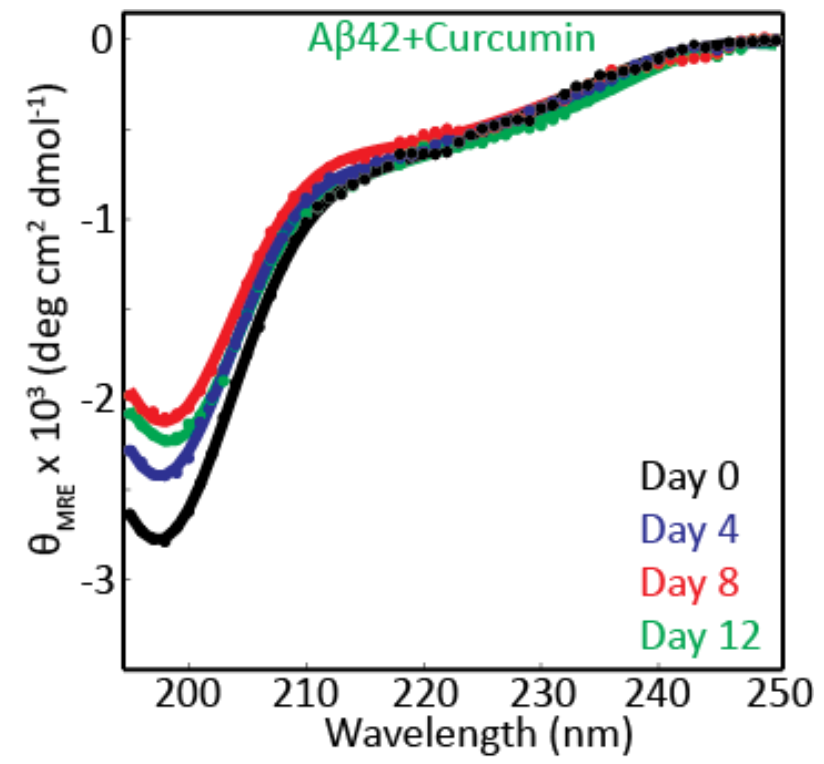

Figure 3.9: CD spectra of AB42 treated with curcumin during a 12-day incubation period; Day 0 (black), Day 4 (blue), Day 8 (red), and Day 12 (green). 
Figure 3.10 shows the dUVRR spectra of the $A \beta$ protein treated with curcumin on Days 0 and 12. Difference spectra are needed to observe any changes that are occurring. The $F / Y$ modes at 1589 and $1611 \mathrm{~cm}^{-1}$ are visibly different between Days 0 and 12 .

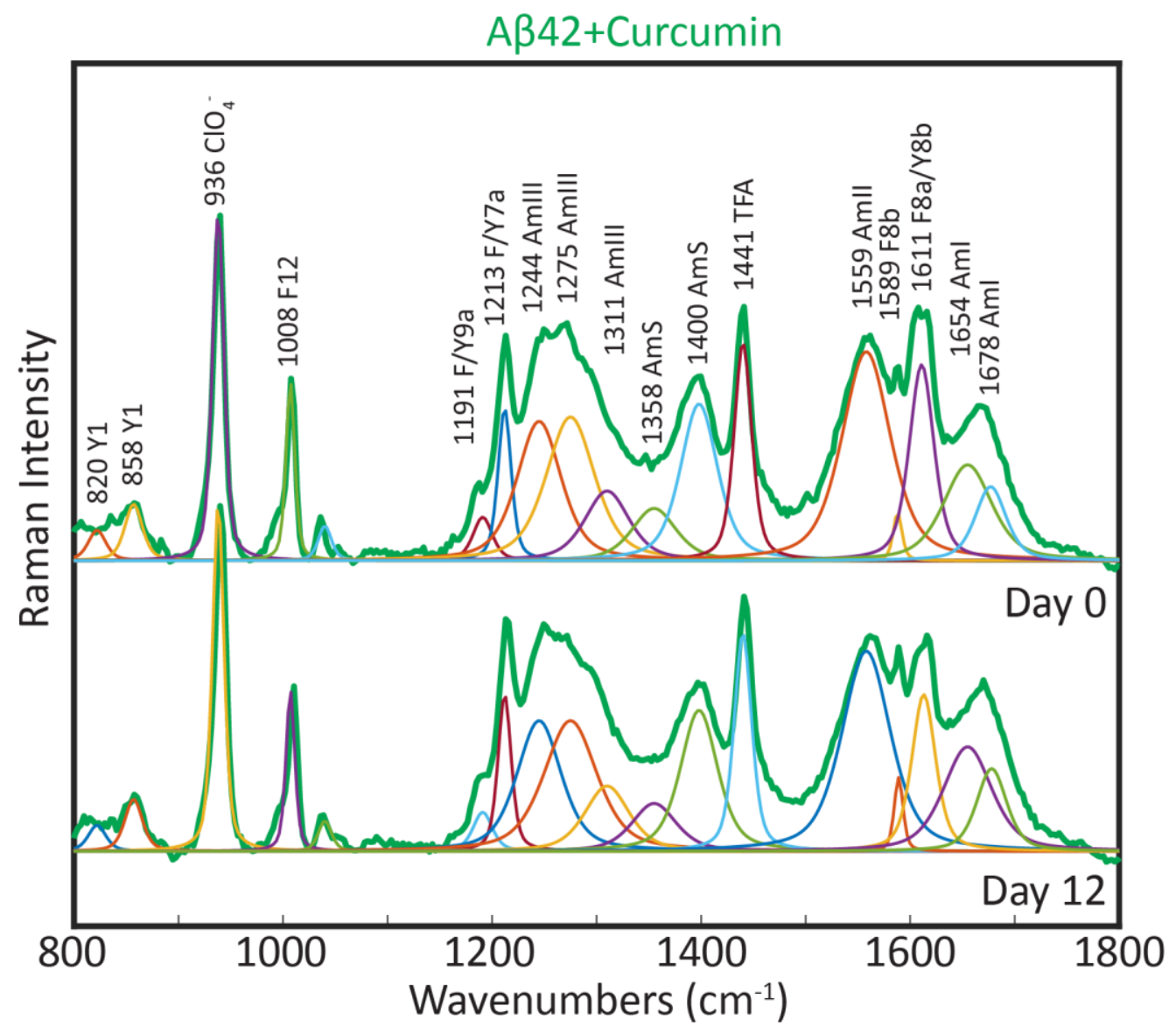

Figure 3.10: Deconvoluted dUVRR spectra of AB42 treated with curcumin on Days 0 and 12 (green). Deconvolution of the individual amide and aromatic modes was performed and those peaks are shown under each spectra. 
Figure 3.11 shows the overlaid spectra of curcumin treated and untreated $A \beta$ samples on Days 0 and 12. The differences between the dUVRR spectra of the untreated and treated $A \beta$ samples on Day 0 are predominately limited to the aromatic ring modes at 1213,1589 , and $1611 \mathrm{~cm}^{-1}$. On Day 12 , the $A \beta$ sample differs from the curcumintreated sample spectrum in the amide I, II, and III modes. Overall, the amide I, II, and III modes are less intense.

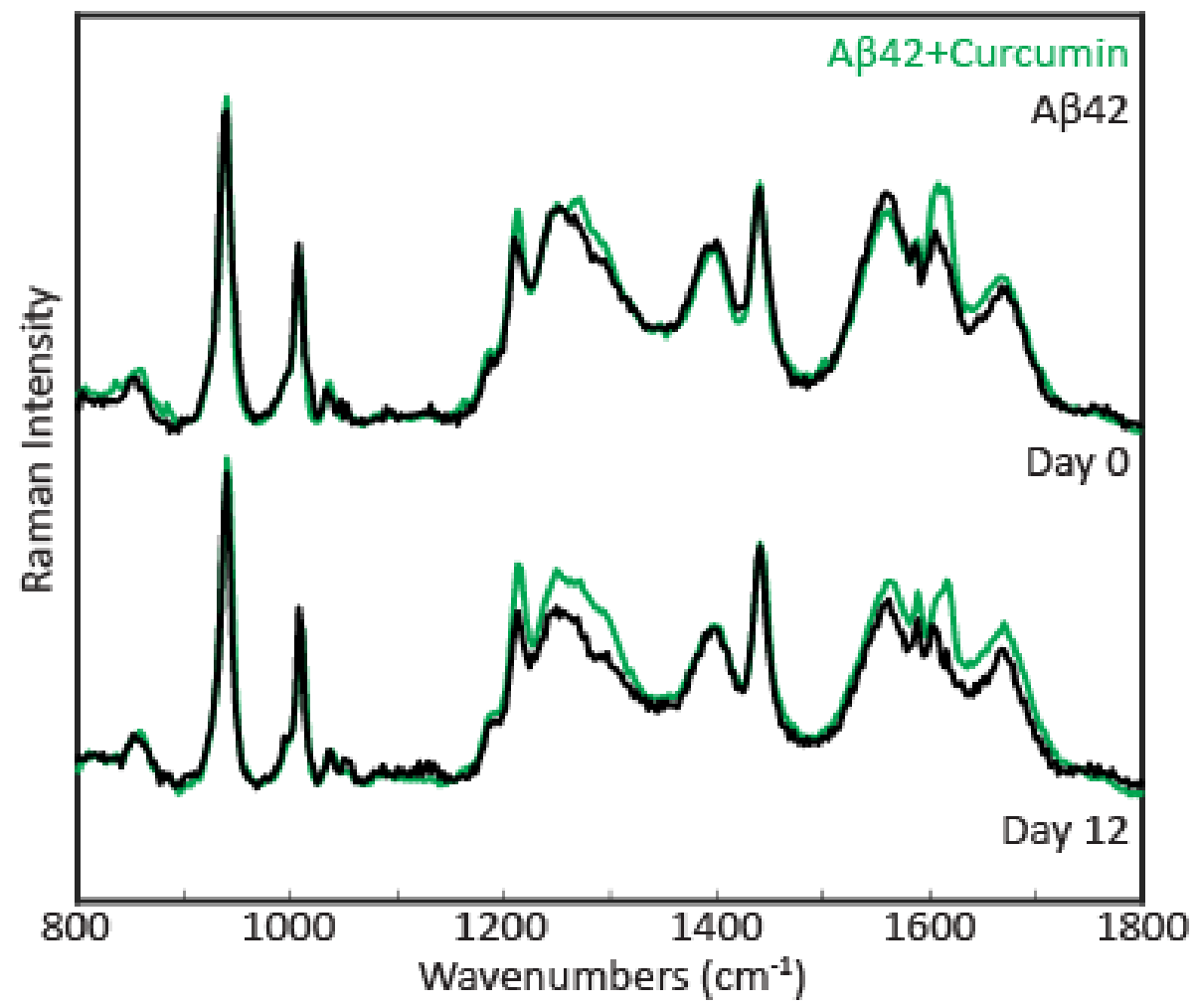

Figure 3.11: DUVRR spectra of AB42 (black) overlaid with AB42 treated with curcumin (green) on Days 0 and 12. 
Figure 3.12 shows the difference between the untreated $A \beta$ protein and the curcumin treated $A \beta$ dUVRR spectra on Days 0 and 12. Initially on Day 0 , the fitted aromatic modes are more intense in the spectrum of the treated sample. By Day 12 , differences in the amide regions are observed due to differences in the structure of the protein between the untreated and curcumin-treated samples.

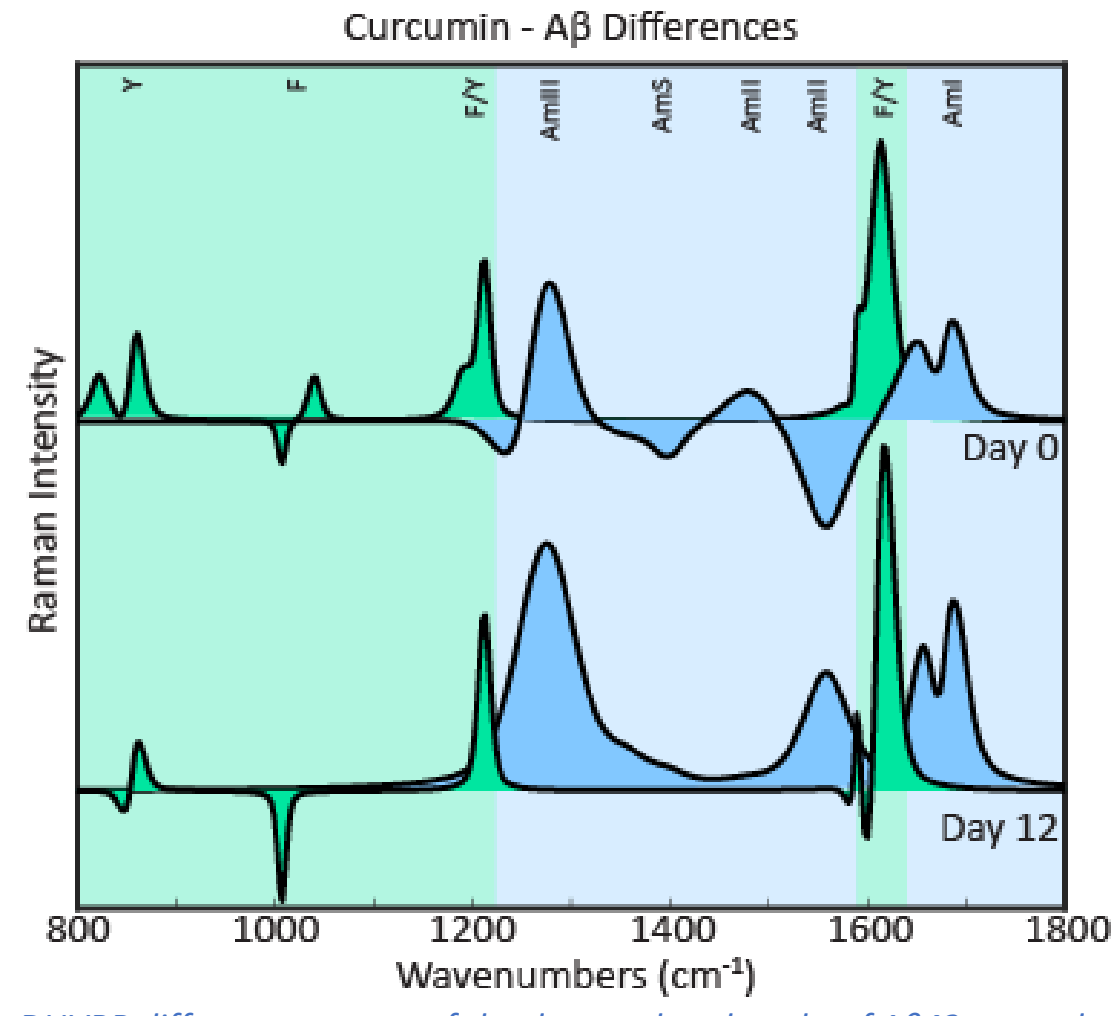

Figure 3.12: DUVRR difference spectra of the deconvoluted peaks of AB42 treated with curcumin and the untreated $A B 42$ on Day 0 and Day 12. The amide modes are shown in blue and the aromatic modes are shown in green. 


\subsubsection{Rosmarinic Acid}

Figure 3.13 shows the $C D$ spectra of rosmarinic acid-treated $A \beta$ during the $12-$ day incubation period. On Day 0 , the protein is disordered in structure, as indicated by the minimum at $197 \mathrm{~nm}$. Although the protein remains disordered in structure over time, there is a loss of intensity at $197 \mathrm{~nm}$, and a minimum at $217 \mathrm{~nm}$ begins to develop.

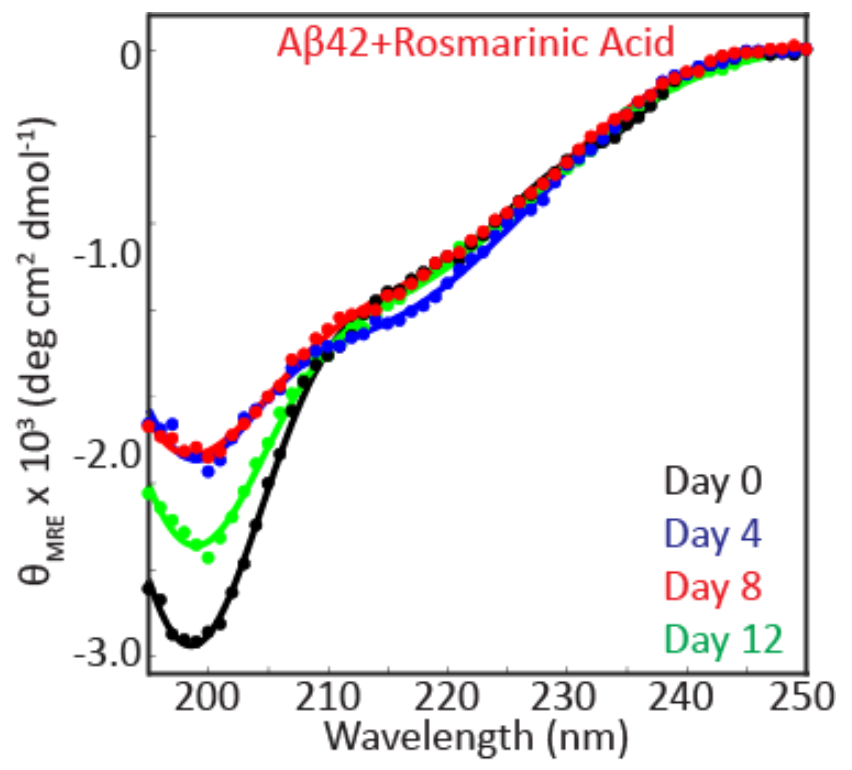

Figure 3.13: CD spectra of $A B 42$ treated with rosmarinic acid during a 12-day incubation period; Day 0 (black), Day 4 (blue), Day 8 (red), and Day 12 (green). 
Figure 3.14 shows the dUVRR spectra of the $A \beta$ protein treated with rosmarinic acid on Days 0 and 12. There is a visible loss of intensity in the amide III mode at 1275 $\mathrm{cm}^{-1}$ and the amide I mode at 1654 and $1678 \mathrm{~cm}^{-1}$. There are also changes in the aromatic modes at 1586 and $1608 \mathrm{~cm}^{-1}$.

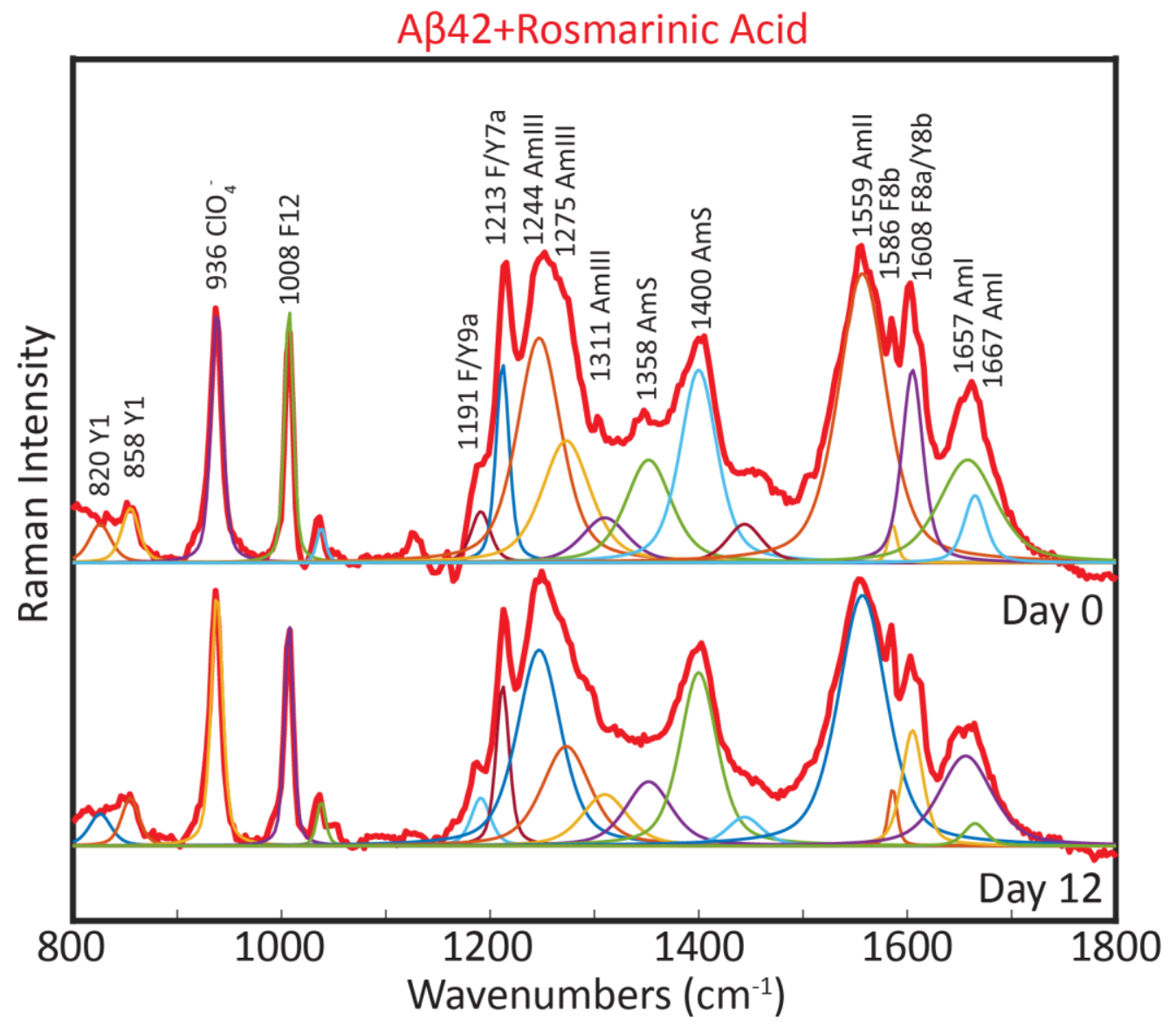

Figure 3.14: Deconvoluted dUVRR spectra of AB42 treated with rosmarinic acid on Days 0 and 12 (red). Deconvolution of the individual amide and aromatic modes was performed and those peaks are shown under each spectra. 
Figure 3.15 shows the overlaid spectra of $A \beta$ treated with rosmarinic acid protein and the untreated protein sample on Days 0 and 12 . The most pronounced difference between the untreated and treated $A \beta$ spectra is the peak in the amide III region around $1295 \mathrm{~cm}^{-1}$. Loss of intensity in this region without a concurrent decrease in the amide III intensity at $1244 \mathrm{~cm}^{-1}$, may suggest a greater proportion of $\beta$-structure in the samples treated with rosmarinic acid. The strong feature at $1441 \mathrm{~cm}^{-1}$ arises from TFA, as mentioned earlier. Addition of rosmarinic acid also results in a decrease in the amide I mode at 1657 and $1667 \mathrm{~cm}^{-1}$ and the $F / Y$ modes at 1586 and $1608 \mathrm{~cm}^{-1}$.

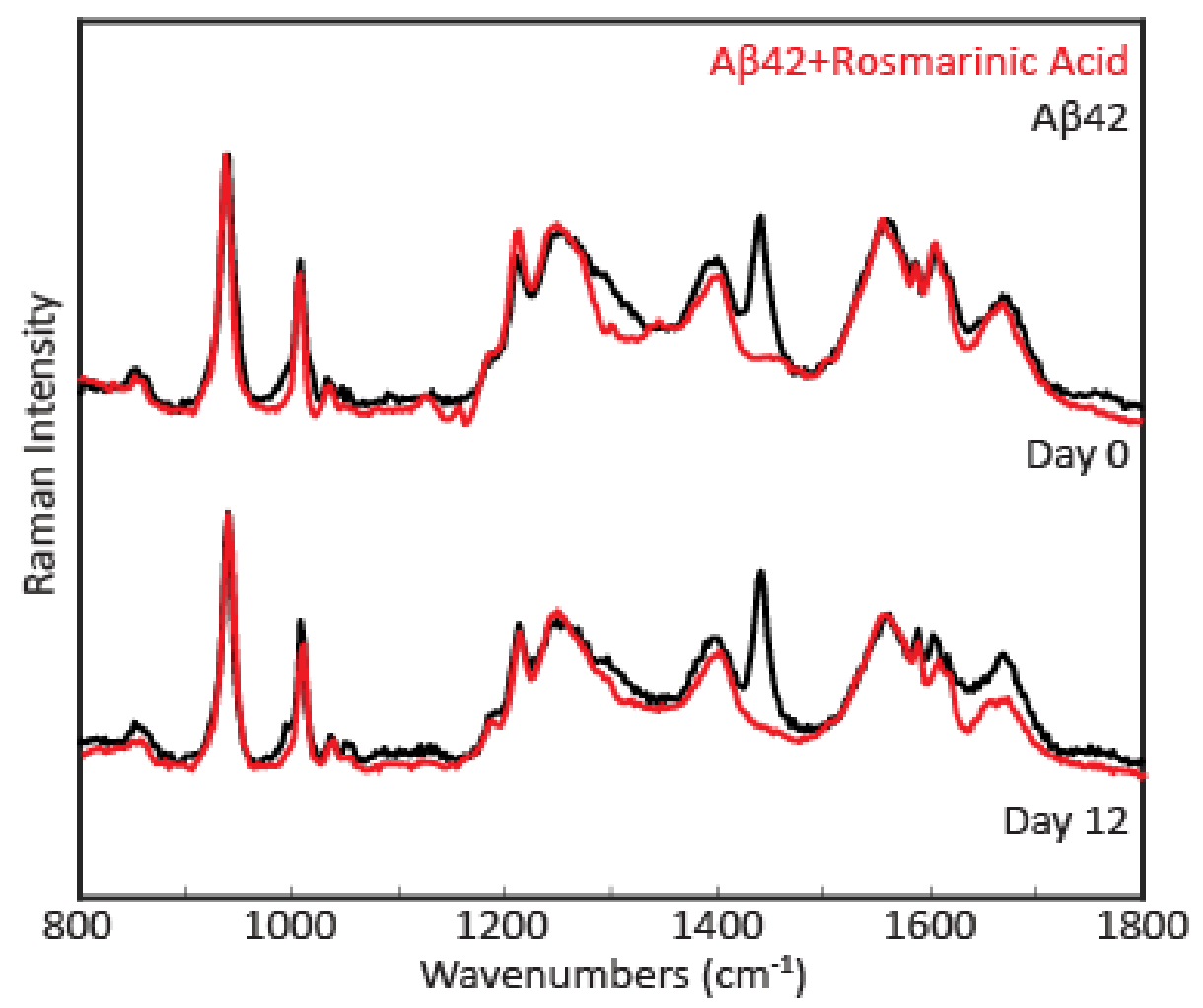

Figure 3.15: DUVRR spectra of AB42 (black) overlaid with AB42 treated with rosmarinic acid (red) on Days 0 and 12. 
Figure 3.16 shows the differences between the dUVRR spectra of the untreated $A \beta$ protein and rosmarinic acid-treated $A \beta$ on Days 0 and 12 . Initially, the differences are predominantly located in the amide regions. Specifically, the amide III band at $1295 \mathrm{~cm}^{-1}$ appear lower in intensity suggesting a structural difference. By Day 12 , the amide III and II modes are stronger in the dUVRR spectrum of the treated sample. Small differences in the $\mathrm{F} / \mathrm{Y}$ modes at 1586 and $1608 \mathrm{~cm}^{-1}$ and the $\mathrm{F}$ mode at $1008 \mathrm{~cm}^{-1}$ are also observed upon rosmarinic acid treatment of $A \beta$.

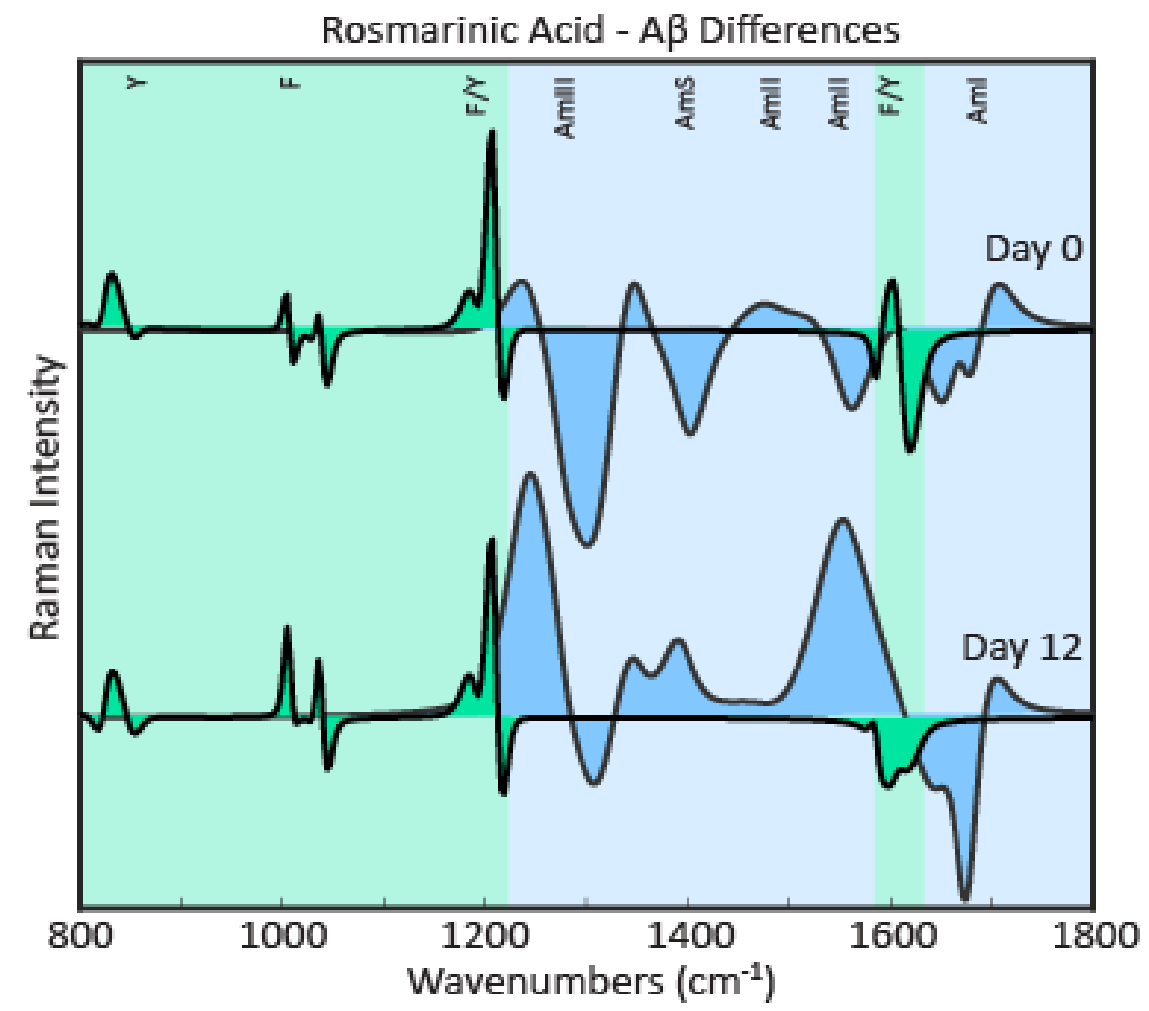

Figure 3.16: DUVRR difference spectra of the deconvoluted peaks of AB42 treated with rosmarinic acid and the untreated AB42 on Day 0 and Day 12. The amide modes are shown in blue and the aromatic modes are shown in green. 


\subsection{Discussion}

As the polarity of the polyphenolic linker increased, the $A \beta$ protein had a greater tendency to form a $\beta$-structure when exposed to polyphenols. Thus more non-polar linkers between the phenol rings should promote disordered structure. A more polar linker leads to the reduction of disordered content in $A \beta$ and an increase in $\beta$-sheet structure. This was first observed by analyzing the $C D$ spectra of $A \beta$ with each polyphenol. Each polyphenol-treated $A \beta$ samples starts off predominantly disordered protein structure on Day 0. The Day 0 CD spectra have the most intense minima at 197 $\mathrm{nm}$, which was observed throughout the 12-day incubation period. The CD spectra of all the polyphenol-treated $A \beta$ samples become less intense over time, but by differing amounts. The CD spectra indicate the nordihydroguaiaretic acid-treated $A \beta$ changes structure only a very small amount and then the structure stabilizes. When curcumin was added to the sample, $A \beta^{\prime}$ 's structure remained disordered, but its structure slowly changed throughout the 12-day incubation period. Rosmarinic acid slowed but did not prevent formation of $\beta$-structure.

Previous studies have shown that when a protein goes from a disordered to $\beta$ sheet structure, changes in the dUVRR spectrum are minimal. There can be an overall loss of intensity, seen mainly in the amide modes, if aggregates form. ${ }^{[15]}$ Also, a blue shift of the amide $S$ mode in the dUVRR spectra can indicate $\beta$-sheet formation from disordered structure. ${ }^{[15]}$ This shift is observed in dUVRR spectra of $A \beta$ in the presence of polyphenols with linkers of increasing polarity (Figure 3.17). The Day 12 dUVRR spectra of $A \beta$ with each polyphenol are stacked to show the peak shift. The 
nordihydroguaiaretic acid-treated protein has the lowest peak maxima at $1394 \mathrm{~cm}^{-1}$, followed by the curcumin-treated protein at $1397 \mathrm{~cm}^{-1}$, and then the rosmarinic acidtreated protein, which occurs at $1405 \mathrm{~cm}^{-1}$. The CD spectra of the rosmarinic acidtreated $A \beta$ changed the most over time. Whereas the $C D$ spectra of the nordihydroguaiaretic acid-treated $A \beta$ remained stable, indicating the protein remained in a stable disordered structure over the 12-day incubation period. Thus, the dUVRR and CD data correlate well.

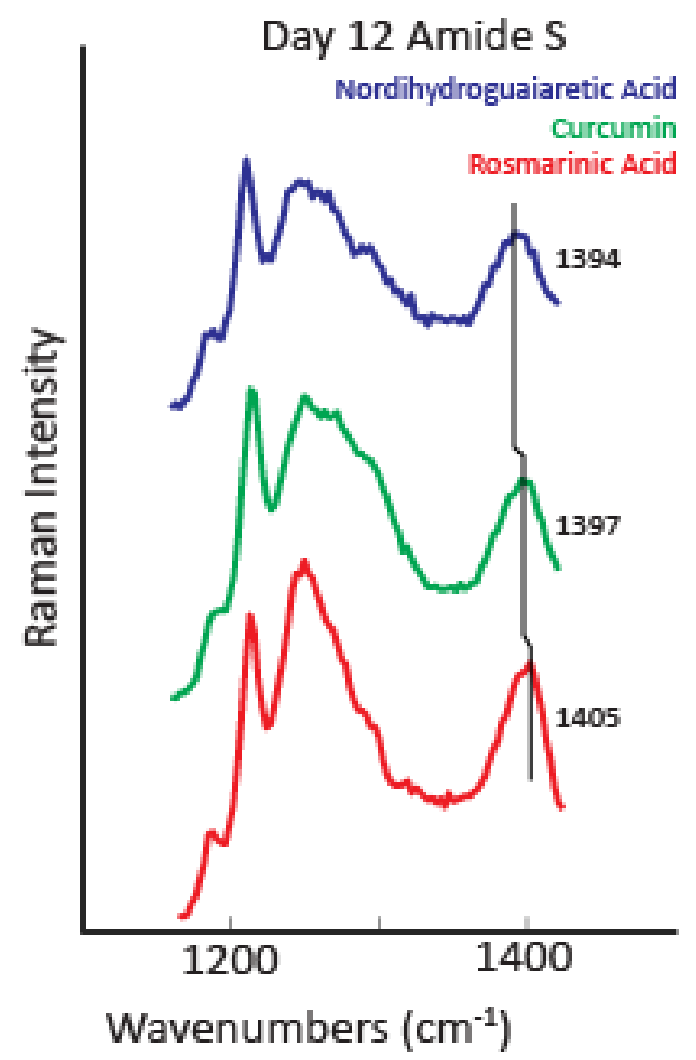

Figure 3.17: Blue shift of the amide $S$ mode of $A B$; nordihydroguaiaretic acid (blue), curcumin (green), and rosmarinic acid (red) treatments.

DUVRR spectroscopy is useful for observing protein conformational changes, but the aromatic modes in dUVRR spectra are also useful for monitoring changes in the environment of aromatic residues within a protein. The two aromatic amino acids 
present in $A \beta$ are $Y$ and $F$. For all polyphenol-treated $A \beta$ samples, the $Y$ mode at $820 \mathrm{~cm}^{-1}$ in dUVRR spectra decreased as compared to untreated $A \beta$ samples. The $Y$ mode at 858 $\mathrm{cm}^{-1}$ also decreases to the greatest extent with nordihydroguaiaretic acid treatment, whereas the $Y$ mode at $1608 \mathrm{~cm}^{-1}$ decreases with all of the polyphenols tested. The more polar the chain linker, the more dramatic the observed decrease was observed. Another mode that follows this polarity trend is the $\mathrm{F}$ mode at $1008 \mathrm{~cm}^{-1}$, for which only nordihydroguaiaretic acid, the least polar polyphenol, caused an increase in its intensity. Curcumin treatment caused a small decrease in this $\mathrm{F}$ mode, whereas and rosmarinic acid treatment caused a large decrease in the F mode intensity as compared to untreated $A \beta$. Among the polyphenols tested, the $Y / F$ mode at $1213 \mathrm{~cm}^{-1}$ decreased the most dramatically when rosmarinic acid was added.

In general, the $Y$ modes decreased in intensity over time when $A \beta$ has been treated with polyphenolic compounds. Polyphenols containing a more polar chain linker, i.e., curcumin and rosmarinic acid, causes decreases in the F mode intensity, while a nonpolar chain linker as in nordihydroguaiaretic acid caused an increase in this mode. Increasing the polarity of the chain linker results in a greater change in the environment of the aromatic residues of the protein. This is seen in Figure 3.18, where $A \beta$ samples treated with rosmarinic acid seem to have the largest differences in the aromatic regions. 


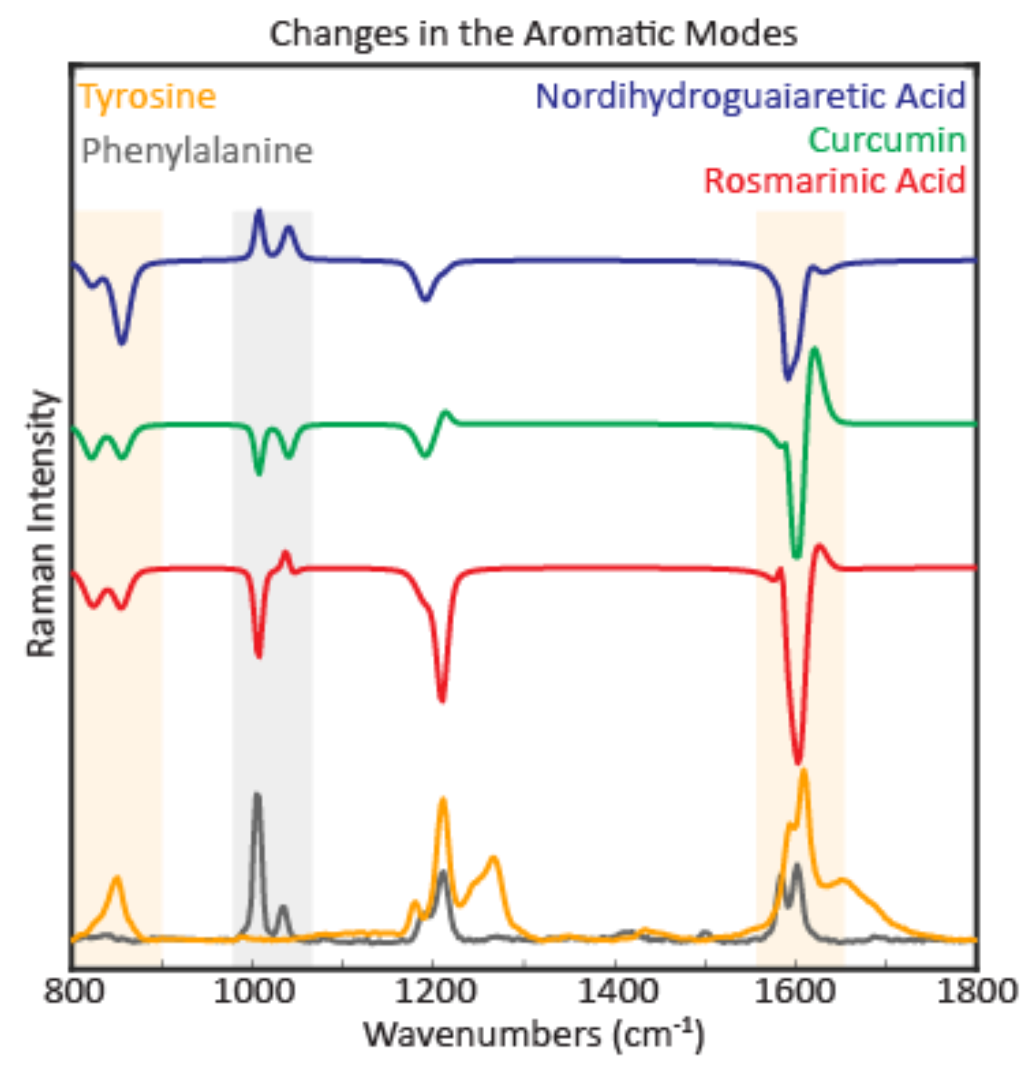

Figure 3.18: Changes over the 12-day incubation period in the individual aromatic modes of the dUVRR spectra. Nordihydroguaiaretic acid (blue), curcumin (green), and rosmarinic acid (red) are shown, as well as spectra of the aromatics tyrosine (orange) and phenylalanine (grey). 


\subsection{References}

1. Lakey-Beitia, J.; Berrocal, R.; Rao, K. S.; Durant, A. A., Polyphenols as therapeutic molecules in Alzheimer's disease through modulating amyloid pathways. Mol Neurobiol 2015, 51 (2), 466-479.

2. Ono, K.; Yoshiike, Y.; Takashima, A.; Hasegawa, K.; Naiki, H.; Yamada, M., Potent antiamyloidogenic and fibril-destabilizing effects of polyphenols in vitro: implications for the prevention and therapeutics of Alzheimer's disease. J Neurochem 2003, 87 (1), 172-181.

3. Shoval, H.; Lichtenberg, D.; Gazit, E., The molecular mechanisms of the anti-amyloid effects of phenols. Amyloid 2007, 14 (1), 73-87.

4. Porat, Y.; Abramowitz, A.; Gazit, E., Inhibition of amyloid fibril formation by polyphenols: structural similarity and aromatic interactions as a common inhibition mechanism. Chem Biol Drug Des 2006, 67 (1), 27-37.

5. Gazit, E., Mechanisms of amyloid fibril self-assembly and inhibition: Model short peptides as a key research tool. FEBS J 2005, 272, 5971-5978.

6. Gazit, E., Global analysis of tandem aromatic octapeptide repeats: The significance of the aromatic-glycine motif. Bioinformatics 2002, 18 (6), 880-883.

7. Porat, Y.; Mazor, Y.; Efrat, S.; Gazit, E., Inhibition of islet amyloid polypeptide fibril formation: a potential role for heteroaromatic interactions. Biochem 2004, 43, 14454-14462.

8. Azriel, R.; Gazit, E., Analysis of the minimuml amyloid-forming fragment of the islet amyloid polypeptide. An experimental support for the key role of the phenylalanine residue in amyloid formation. J Biol Chem 2001, 276 (36), 34156-24161.

9. Hudson, S. A.; Ecroyd, H.; Dehle, F. C.; Musgrave, I. F.; Carver, J. A., (-)-Epigallocatechin-3-gallate (EGCG) maintains kappa-casein in its pre-fibrillar state without redirecting its aggregation pathway. J Mol Biol 2009, 392 (3), 689-700.

10. Convertino, M.; Pellarin, R.; Catto, M.; Carotti, A.; Caflisch, A., 9,10-Anthraquinone hinders betaaggregation: how does a small molecule interfere with Abeta-peptide amyloid fibrillation? Protein Sci 2009, 18 (4), 792-800. 
11. Lashuel, H. A.; Hartley, D. M.; Balakhaneh, D.; Aggarwal, A.; Teichberg, S.; Callaway, D. J., New class of inhibitors of amyloid-beta fibril formation. Implications for the mechanism of pathogenesis in Alzheimer's disease. J Biol Chem 2002, 277 (45), 42881-42890.

12. Arteaga, S.; Andrade-Cetto, A.; Cárdenas, R. Larrea tridentate (Creosote bush), an abundant plant of Mexican and US-American deserts and its metabolite nordihydroguaiaretic acid. J Ethnopharmacol 2005, 98 (3), 231-239.

13. Gupta, S. C.; Kismali, G.; Aggarwal B. B. Curcumin, a component of turmeric: from farm to pharmacy. Biofactors 2013, 39 (1), 2-13.

14. al-Sereiti, M. R.; Abu-Amer, K. M.; Sen, P. Pharmacology of rosemary (Rosmarinus officinalis Linn.) and its therapeutic potentials. Indian J Exp Biol 1999, 37 (2), 124-130.

15. Oladepo, S. A.; Xiong, K.; Hong, Z.; Asher, S. A.; Handen, J.; Lednev, I. K. UV resonance Raman investigations of peptide and protein structure and dynamics. Chem Rev 2012, 112, 2604-2628. 


\section{Chapter IV: Increasing Ring Substituents Destabilizes Pre-formed Amyloid- $\beta$ Fibrils}

\subsection{Abstract}

Stilbenoid compounds have common structural components consisting of two phenolic rings with hydroxyl group substituents and a chain linker between the rings. Hydroxyl groups on polyphenolic rings not only facilitate hydrogen bonding with the protein backbone, but also provide the compound with the ability to be an antioxidant. The antioxidant capacity increases with the number of hydroxyl substituents. This study investigated the ability of stilbenoid compounds differing in the number of hydroxyl substituents, to destabilize pre-formed $A \beta$ fibrils. The polyphenols diethylstilbestrol, resveratrol, and piceatannol are structurally similar, but differ in the number of hydroxyl group substituents, i.e., two, three, and four, respectively. It was found that with increased number of hydroxyl ring substituents, pre-formed $A \beta$ fibrils were destabilized more readily by the polyphenol. 


\subsection{Introduction}

Hydroxyl group substituents on polyphenolic rings allow for essential binding to the protein through hydrogen bonds from the polyphenol to the amide rich backbone of the protein. ${ }^{[1-3]}$ Studies have shown that the more hydroxyl groups, the higher the antioxidant activity. ${ }^{[4]}$ Ono et al. compared four flavonoid compounds (myricetin, morin, quercetin, and kaempferol ) that have similar structures and differ only in the numbers of hydroxyl substituents. It was found that decreasing the number of hydroxyl substituents decreased the anti-amyloidogenic and fibril-destabilizing activities of the polyphenol. ${ }^{[4]}$ Polyphenols can act on $A \beta$ aggregation at three different stages; initial formation of fibrils, extension of formed fibrils, and destabilization of pre-formed $A \beta$ fibrils. A polyphenol may act at only one of these stages or possibly at all three. ${ }^{[4,5]}$ In Chapter 2, it was shown that increasing the number of hydroxyl substituents on polyphenols incubated with $A \beta$ retarded structural changes that promote progression from a disordered to $\beta$-sheet structure. Using the same polyphenols as in Chapter 2 (i.e., diethylstilbestrol, resveratrol, and piceatannol), their effect on destabilization of pre-formed A $\beta$ fibrils was assessed. The polyphenols diethylstilbestrol, resveratrol, and piceatannol shown in Figure 4.1 have an increasing numbers of hydroxyl substituents on their rings, having two, three, and four, respectively. They each have a 2-atom carbon chain between the two rings allowing for a conjugated double bond system. This study aims to examine how the number of hydroxyl group substituents affects the polyphenol's ability to destabilize pre-formed $A \beta$ fibrils. 


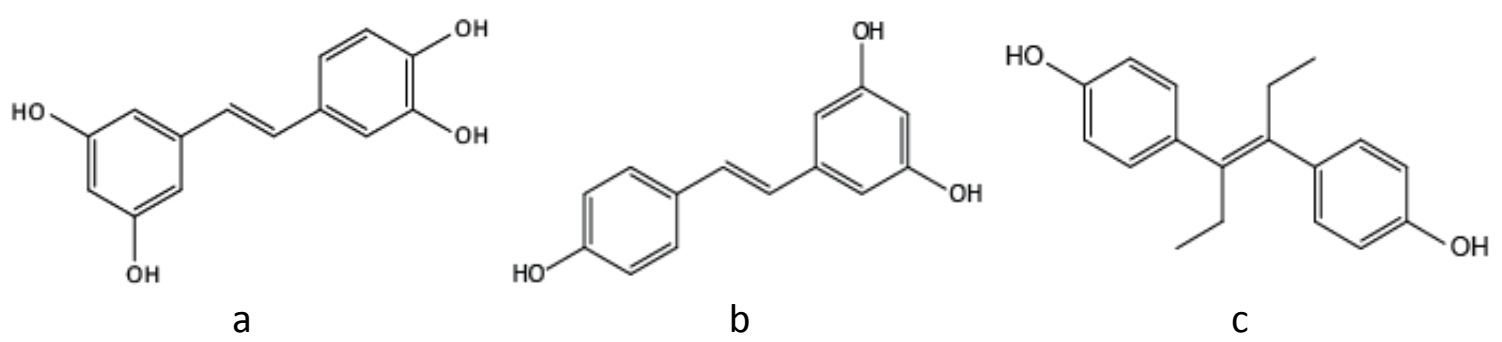

Figure 4.1: Structures of piceatannol (a), resveratrol (b), and diethylstilbestrol (c).

\subsection{Materials and Methods}

\subsubsection{Materials}

A $\beta$ (1-42) (>95\% pure) was purchased from Atlantic Peptides (Scarborough, ME). Ethanol and sodium perchlorate were purchased from Sigma-Aldrich (St. Louis, MO) and used without further purification. Sodium phosphate monobasic, sodium phosphate dibasic, sodium chloride, and cyclohexane were purchased from Fisher Scientific (Pittsburgh, PA). Thioflavin T, resveratrol, piceatannol, and diethylstilbestrol were purchased from Sigma-Aldrich (St. Louis, MO).

\subsubsection{Amyloid- $\beta$ (1-42) Protein Preparation}

$\mathrm{A} \beta$ (1-42) (M.W. $4512.3 \mathrm{kDa}$ ) was dissolved in $10 \mathrm{mM}$ sodium phosphate buffer, $20 \mathrm{mM} \mathrm{NaCl}, \mathrm{pH}$ 7.4, sonicated for $10 \mathrm{~min}$ in an ice bath and centrifuged for 30 minutes at $14,000 \mathrm{rcf}$ and $4^{\circ} \mathrm{C}$ (Centrifuge $5804 \mathrm{R}$, Eppendorf AG, Hamburg Germany). The concentration was estimated using the extinction coefficient of tyrosine, which is 1,280 $\mathrm{M}^{-1} \mathrm{~cm}^{-1}$ at $280 \mathrm{~nm}$ using a Varian Cary Bio 50 UV-Vis spectrophotometer (Varian, Palo Alto, $\mathrm{CA})^{[6]}$. Peptide concentrations ranged from $70-80 \mu \mathrm{M}$ and were normalized in all the spectral processing. $A \beta$ was prepared and incubated at $37^{\circ} \mathrm{C}$ for a 12 -day time period. During this time, samples were characterized every two days by CD spectroscopy. Stock solutions of each polyphenolic compound were prepared in 
ethanol at a concentration of $5 \mathrm{mM}$. Polyphenolic compounds were then added to the protein solutions on Day 6, mid-way through the incubation period, for a final concentration of $25 \mu \mathrm{M}$.

\subsubsection{Circular Dichroism (CD)}

A Jasco J-815 CD Spectropolarimeter (Jasco, Easton, MD) was used to collect CD spectra in the range of $190-250 \mathrm{~nm}$. A scan speed of $50 \mathrm{~nm} / \mathrm{min}$ with a response time of $4 \mathrm{sec}$ was used. Five spectra were averaged for each sample. A $1 \mathrm{~mm}$ optical path length quartz cuvette (Hellman, Plainview, NY) was employed for all measurements.

\subsubsection{Thioflavin T Assays}

Fluorescence was measured on a Cary Eclipse spectrometer (Varian, Palo Alto, CA). A $3 \mathrm{~mm}$ path length quartz cuvette (Hellman Plainview, NY) was employed for all measurements. The emission spectra were measured using an excitation wavelength of $450 \mathrm{~nm}$, and the fluorescence was collected from 470-700 nm.

\subsubsection{Data Analysis}

Data analysis was carried out in the MATLAB R206b (MathWorks, Natick, MA) environment. For $\mathrm{CD}$, all spectra were averaged and the background buffer spectrum was subtracted from the raw data. The mean residue ellipticity $\left(\theta_{\mathrm{MRE}}\right)$ was calculated using Equation 1 with $\vartheta$ being the raw ellipticity from the measurement (degrees), $M W$ being the molecular weight of the protein $(\mathrm{g} / \mathrm{mol}), n$ being the number of protein residues, $d$ being the path length of the cell $(\mathrm{cm})$, and $C$ being the concentration of the sample (M). ${ }^{[6-8]}$ All spectra were the average of triplicate trials.

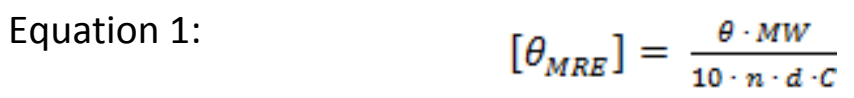




\subsection{Results}

\subsubsection{AB Untreated}

$A \beta$ samples were prepared and incubated for 12 days. Figure 4.2 shows the $C D$ spectra of $A \beta$ incubated at $37^{\circ} \mathrm{C}$ over a 12 -day period. On Day 0 , the $C D$ spectrum indicates that the protein is predominantly disordered. By Day 6, the protein was beginning to form $\beta$-sheet structure. The ThT assays indicated that the increase in $\beta$ sheet formation was associated with an increase in fibrils which is correlated with an increase in ThT fluorescence intensity (Figure 4.3). The CD spectra of $A \beta$ on Days 8-12 indicate the complete loss of any disordered content. A single minimum present around $217 \mathrm{~nm}$ indicated that the peptide is predominantly $\beta$-sheet structured. There is a general loss in the overall intensity of the CD spectra in a manner that would suggest that the protein concentration may be decreasing over time. It is possible that more of the protein starts to form $\beta$-sheet structures, small oligomers form. These oligomers can be soluble or insoluble. The insoluble oligomers may fall out of solution causing the spectrum to lose intensity in all regions. 


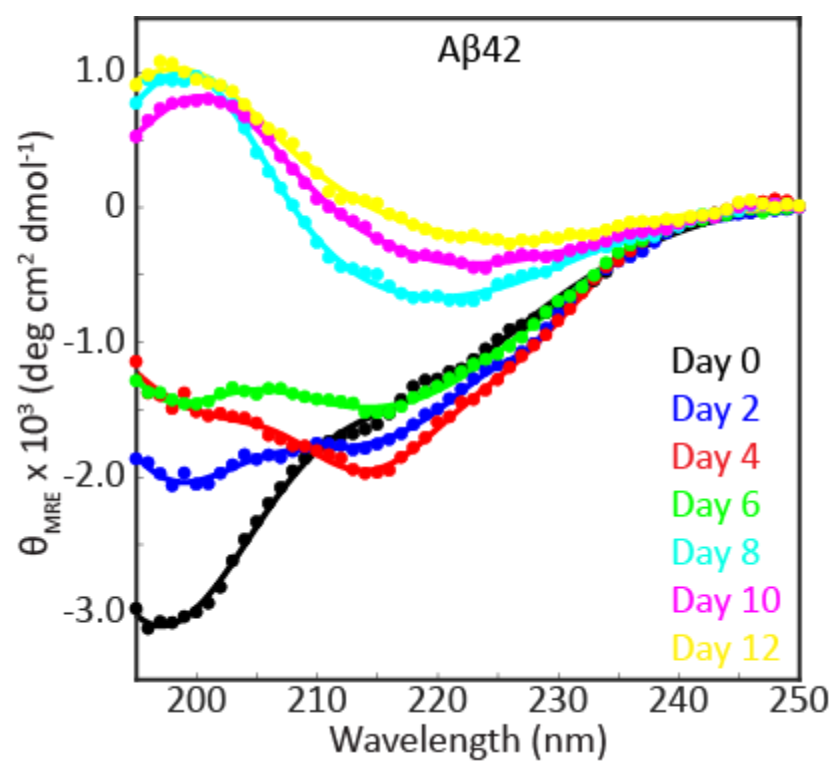

Figure 4.2: CD spectra of AB42 over a 12-day incubation period; Day 0 (black), Day 2 (blue), Day 4 (red), Day 6 (green), Day 8 (cyan), Day 10 (pink), and Day 12 (yellow).

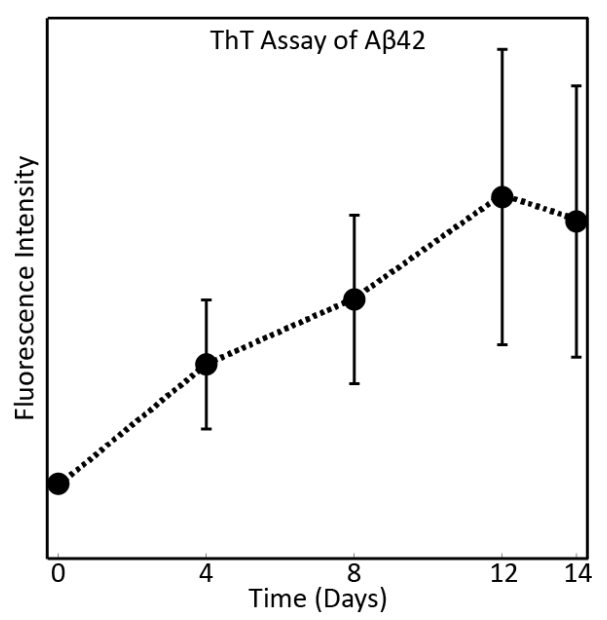

Figure 4.3: ThT fluorescence of AB42 fibrillization over a 14-day incubation period. 


\subsubsection{Piceatannol}

Piceatannol, which has four hydroxyl substituents, shifted the structure of $A \beta$ from $\beta$-sheet to disordered which is evidenced by the re-emergence of a minima at 197 $\mathrm{nm}$ in the CD spectra (Figure 4.4). The CD spectra did not change dramatically over the remaining 6 days, though the minima at $217 \mathrm{~nm}$ decreased in intensity slightly indicating a small progressive loss in the remaining $\beta$-sheet content.

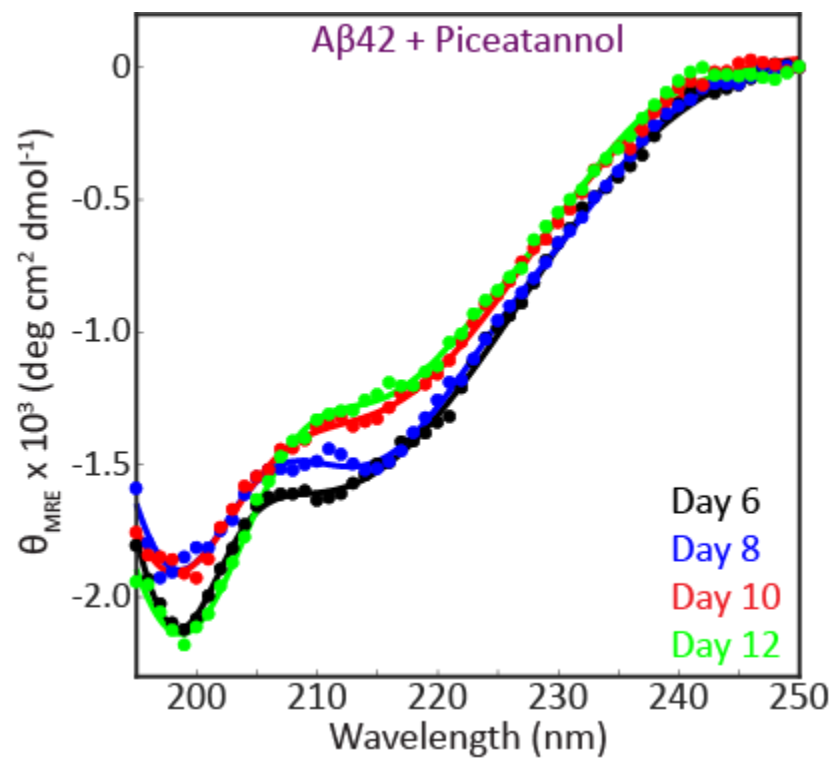

Figure 4.4: $C D$ spectra of $A B 42$ treated with piceatannol midway during a 12-day incubation period. The AB protein was treated with piceatannol on Day 6 (black). The protein continued to incubate through Day 8 (blue), Day 10 (red), and Day 12 (green). 


\subsubsection{Resveratrol}

Resveratrol, which has three hydroxyl substituents, also shifted the structure of $A \beta$ from $\beta$-sheet to disordered, based on the appearance of a minima at $197 \mathrm{~nm}$ in the CD spectrum (Figure 4.5). Although the addition of piceatannol and resveratrol had very similar immediate effects on $A \beta^{\prime}$ 's structure, the minima at $197 \mathrm{~nm}$ completely disappeared between Days 10 and 12 indicating the resveratrol did not prevent $\beta$-sheet formation after destabilizing fibrils. These data indicate that resveratrol can destabilize the $\beta$-sheet structure in the fibrils or protofibrils but is not able to prevent their reformation. It is possible that oxidation of resveratrol makes it less effective at later points. In Chapter 2, it was observed the resveratrol delayed but did not prevent $\beta$-sheet formation, which is consistent with these results.

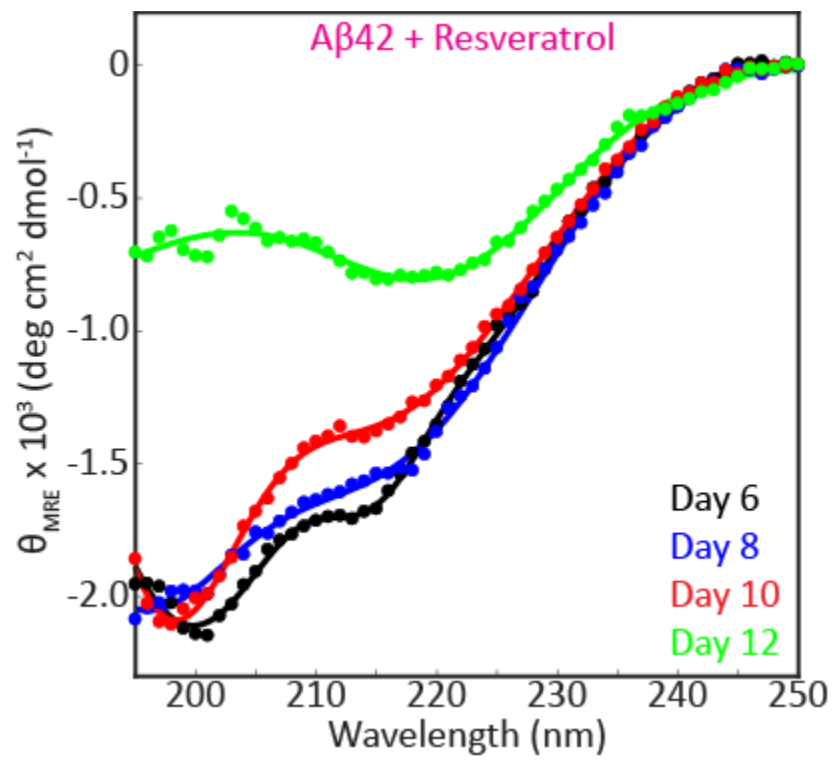

Figure 4.5: $C D$ spectra of $A B 42$ treated with resveratrol midway during a 12-day incubation period. The $A B$ protein was treated with resveratrol on Day 6 (black). The protein continued to incubate through Day 8 (blue), Day 10 (red), and Day 12 (green). 


\subsubsection{Diethylstilbestrol}

Diethylstilbestrol, which has two hydroxyl substituents, appears to promote $\beta$ sheet formation as evidenced by a stronger minima at $215 \mathrm{~nm}$ (Figure 4.6). On Day 6, with the addition of the polyphenol to the $A \beta$ sample, a very small feature with a minimum at $197 \mathrm{~nm}$ is seen, however this feature is small in comparison to the other minimum at $215 \mathrm{~nm}$. By Day 8 , this feature is no longer present in the CD spectrum and the protein continues to stay predominantly $\beta$-sheet in structure. The overall loss in intensity of the spectra is similar to that of the untreated $A \beta$ sample most likely due to the formation of insoluble small oligomers.

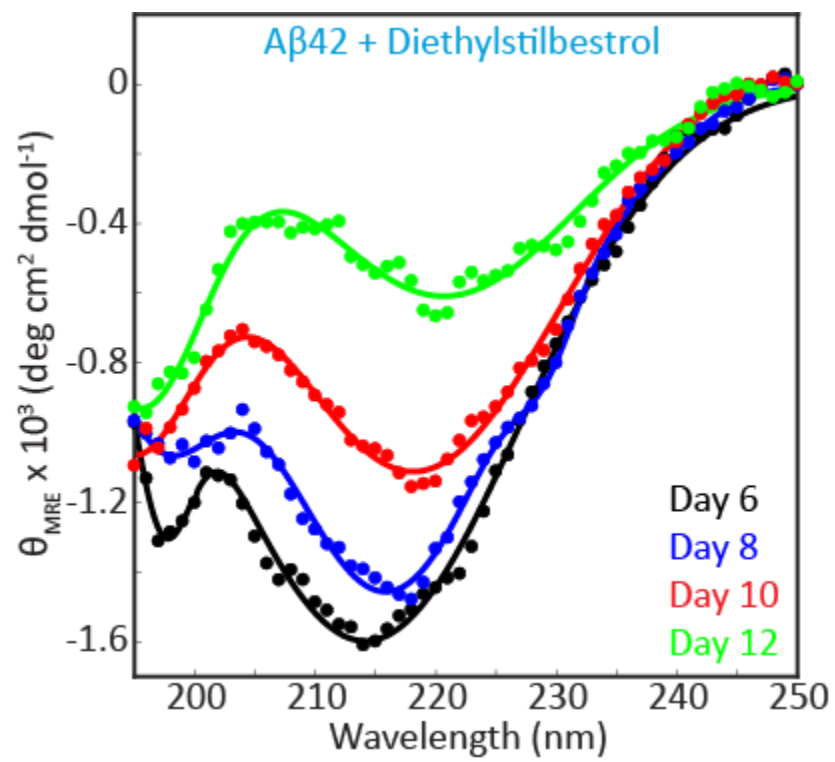

Figure 4.6: CD spectra of AB42 treated with diethylstilbestrol midway during a 12-day incubation period. The AB protein was treated with diethylstilbestrol on Day 6 (black). The protein continued to incubate through Day 8 (blue), Day 10 (red), and Day 12 (green).

\subsection{Discussion}

As the number of hydroxyl substituents is increased on the rings, the polyphenol is more capable of destabilizing the pre-formed $A \beta$ fibrils. Piceatannol, with 4 hydroxyl groups, has an immediate effect on the secondary structure, changing the structure of 
the $A \beta$ protein from $\beta$-sheet to disordered. This effect remained throughout the incubation period. Resveratrol, with 3 hydroxyl groups, initially promoted disordered structure in the $A \beta$ protein, however the polyphenol was incapable of preventing the protein from reforming fibrils potentially due to a loss of activity over time.

Diethylstilbestrol, with only 2 hydroxyl groups, had a limited initial effect on the protein's structure. The protein formed predominantly $\beta$-sheet structure similar to the untreated $A \beta$ solution.

Figure 4.7 shows the intensity of the CD spectra of untreated and treated samples at $217 \mathrm{~nm}$. Evaluation of the intensity of the CD spectra at $217 \mathrm{~nm}$, shows that the structure of the piceatannol-treated sample is relatively stable over time. The resveratrol-treated $A \beta$ sample is also is fairly stable with changes in structure occurring after Day 10. The diethylstilbestrol-treated $A \beta$ sample has the most noticeable change in structure when compared to the other two polyphenols. Diethylstilbestrol is the least effective polyphenol in destabilizing pre-formed $A \beta$ fibrils. Thus, increasing the number of hydroxyl substituents, which increases the antioxidant potency of the polyphenol, makes the polyphenol capable of destabilizing pre-formed $A \beta$ fibrils. 


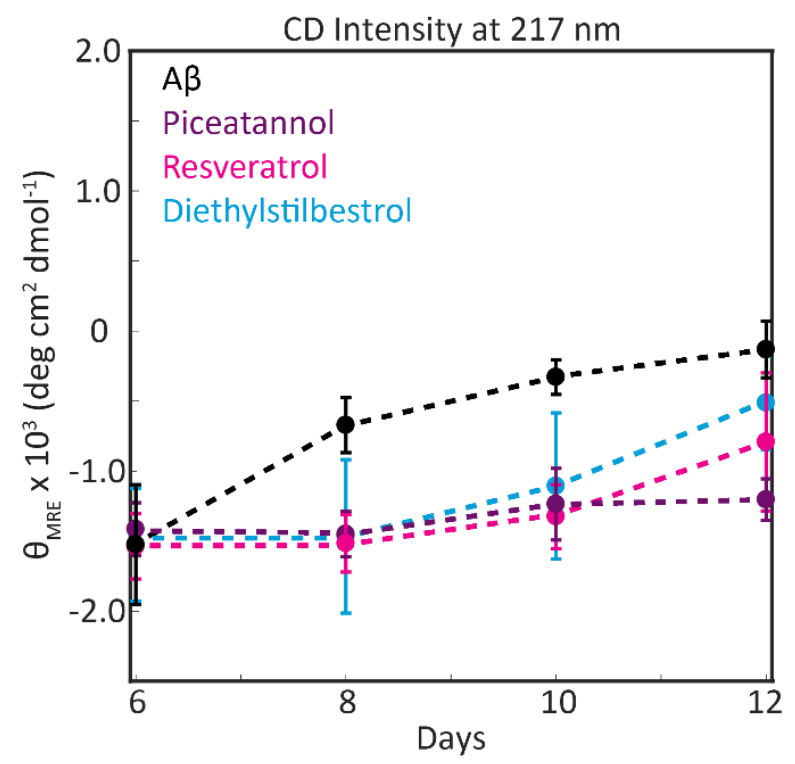

Figure 4.7: Intensity changes at $217 \mathrm{~nm}$ of the polyphenol-treated and untreated AB samples, over a 6-day incubation period; piceatannol (purple), resveratrol (pink), diethylstilbestrol (blue), and $A B$ (black). Error bars are shown based on three repetitive trials. 


\subsection{References}

1. Lakey-Beitia, J.; Berrocal, R.; Rao, K. S.; Durant, A. A., Polyphenols as therapeutic molecules in Alzheimer's disease through modulating amyloid pathways. Mol Neurobiol 2015, 51 (2), 466-479.

2. Porat, Y.; Abramowitz, A.; Gazit, E., Inhibition of amyloid fibril formation by polyphenols: structural similarity and aromatic interactions as a common inhibition mechanism. Chem Biol Drug Des 2006, 67 (1), 27-37.

3. Gauci, A. J. C., M.; Giese, A.; Scerri, C.; Vassallo, V. , Identification of polyphenolic compounds and black tea extract as potent inhibitors of lipid membrane destabilization by A beta(42) aggregates - ResearchGate. J Alz Dis 2011, 24 (4), 767-779.

4. Ono, K.; Yoshiike, Y.; Takashima, A.; Hasegawa, K.; Naiki, H.; Yamada, M., Potent antiamyloidogenic and fibril-destabilizing effects of polyphenols in vitro: implications for the prevention and therapeutics of Alzheimer's disease. J Neurochem 2003, 87 (1), 172-181.

5. Shoval, H.; Lichtenberg, D.; Gazit, E., The molecular mechanisms of the anti-amyloid effects of phenols. Amyloid 2007, 14 (1), 73-87.

6. Wang, M.; JiJi, R.D. Spectroscopic detection of $\beta$-sheet structure in nascent A $\beta$ oligomers. J Biophotonics 2011, 4, 637-644.

7. Simpson, J. V.; Balakrishnan, G.; JiJi, R. D. MCR-ALS analysis of two-way UV resonance Raman spectra to resolve discrete protein secondary structural motifes. Analyst 2009, 134, 138-147.

8. Wang, M.; JiJi, R. D. Resolution of localized small molecule-A $\beta$ interactions by deep-ultraviolet resonance Raman spectroscopy. Biophys Chem 2011, 158, 96-103. 


\section{Chapter V: Increasing Chain Linker Polarity Destabilizes Pre-formed Amyloid- $\beta$ Fibrils}

\subsection{Abstract}

Diaryl polyphenolic compounds have common structural components consisting of two phenolic rings with hydroxyl group substituents and a chain linker between the rings. The chain between the two phenol rings can vary significantly both in length and atom makeup, altering the anti-amyloidgenic properties of the polyphenol. Flavonoid compounds do not contain a chain linker and understanding how the chain linker can affect the activity of linear polyphenols is imperative. The aromatic rings of polyphenols are important for their binding to proteins and their effects on protein aggregation. The type of linker between these rings is also important because it could also affect protein binding and aggregation. The polyphenols nordihydroguaiaretic acid, curcumin, and rosmarinic acid have increasing polarity, respectively, in the chain linker region between the phenolic rings. Each of these polyphenols has four ring substituents and $4-6$ atoms in their chain linker. In this study, it was found that all the polyphenols disrupted and at least partially reversed fibrillization, though no clear trend with polarity of the linker region was observed. 


\subsection{Introduction}

The chain between the two phenol rings can vary significantly both in length and atom makeup. These differences have an important impact on how the polyphenol metabolizes and behaves. ${ }^{[1-2]}$ It is suggested that the optimum chain length between the rings should be 2-6 atoms long. ${ }^{[3]}$ Studies have shown that diaryl polyphenols such as curcumin, are better than flavonoids at inhibiting the amyloidogenic activity of $A \beta$ possibly due to the type of chain linker in between the aromatic rings. ${ }^{[1-2]}$

The aromatic rings are important for polyphenol binding to proteins and their effect on protein aggregation. Aromatic rings of polyphenols provide the necessary hydrophobic interactions with proteins that can interrupt endogenous hydrophobic interactions within the protein which promote aggregation..$^{[3,4-9]}$ The rings of polyphenols have the necessary planarity to bind to $A \beta$ in the hydrophobic groove, interrupting the $\pi$ stacking, and preventing aggregation by intercalating within the $\beta$ sheets. $^{[1,4,10-11]}$ The chain linker polarity determines the polyphenol's overall polarity and its ability to disrupt the hydrophobic interactions necessary for protein aggregation.

The polyphenols nordihydroguaiaretic acid, curcumin, and rosmarinic acid have increasing polarity respectively in the chain linker region between their phenolic rings. Each of these polyphenols has four ring substituents and 4 - 6 atoms in their chain linker. In Chapter 3, the effect of polarity in the chain linker region on the structure of the $A \beta$ protein during the aggregation process was evaluated. It was shown that decreasing the polarity of the chain linker increased the ability of the polyphenol to interfere with $A \beta$ fibrillization. Using the same polyphenols, i.e., nordihydroguaiaretic 
acid, curcumin, and rosmarinic acid, shown in Figure 5.1, it was evaluated whether these molecules can also destabilize pre-formed $A \beta$ fibrils.

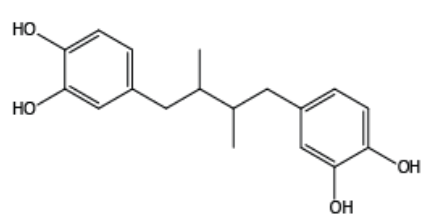

a

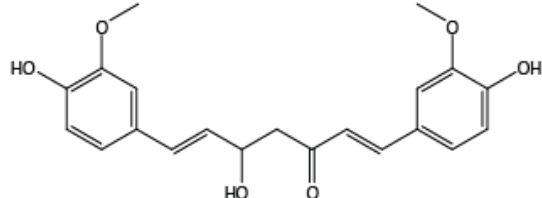

b

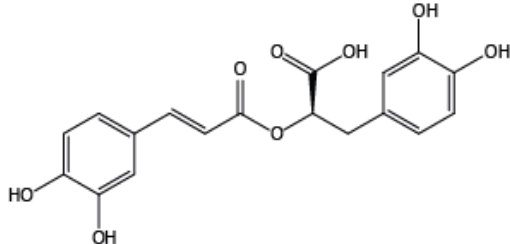

C

Figure 5.1: Structures of nordihydroguaiaretic acid (a), curcumin (b), and rosmarinic acid (c).

\subsection{Materials and Methods}

\subsubsection{Materials}

A $\beta$ (1-42) (>95\% pure) was purchased from Atlantic Peptides (Scarborough, ME). Ethanol and sodium perchlorate were purchased from Sigma-Aldrich (St. Louis, MO) and used without further purification. Sodium phosphate monobasic, sodium phosphate dibasic, sodium chloride, and cyclohexane were purchased from Fisher Scientific (Pittsburgh, PA). Thioflavin T, rosmarinic acid, and nordihydroguaiaretic acid were purchased from Sigma-Aldrich (St. Louis, MO) and curcumin was purchased from Acros Organics (Pittsburgh, PA).

\subsubsection{Methods}

The amyloid- $\beta$ (1-42) protein was prepared and characterized as described in Chapter 4, sections 4.3.2-4.3.4. Circular dichroism (CD) and were analyzed as described in Chapter 4, section 4.3.5. 


\subsection{Results}

\subsubsection{AB Untreated}

The initial $C D$ spectra of the $A \beta$ protein indicated that the protein was predominantly disordered in structure, with a strong minima at $197 \mathrm{~nm}$ (Figure 5.2). By Day 6 , the protein had formed $\beta$-sheet fibrils as evidenced by the characteristic minima at $217 \mathrm{~nm}$ in the CD spectrum and concurrent increase in ThT fluorescence (Figure 5.3). Between Days 8-12 the CD spectra indicate a complete loss of any disordered content as the only minimum present is around $217 \mathrm{~nm}$. The CD spectra generally lose overall intensity in a manner that would suggest that the protein concentration may be decreasing over time.

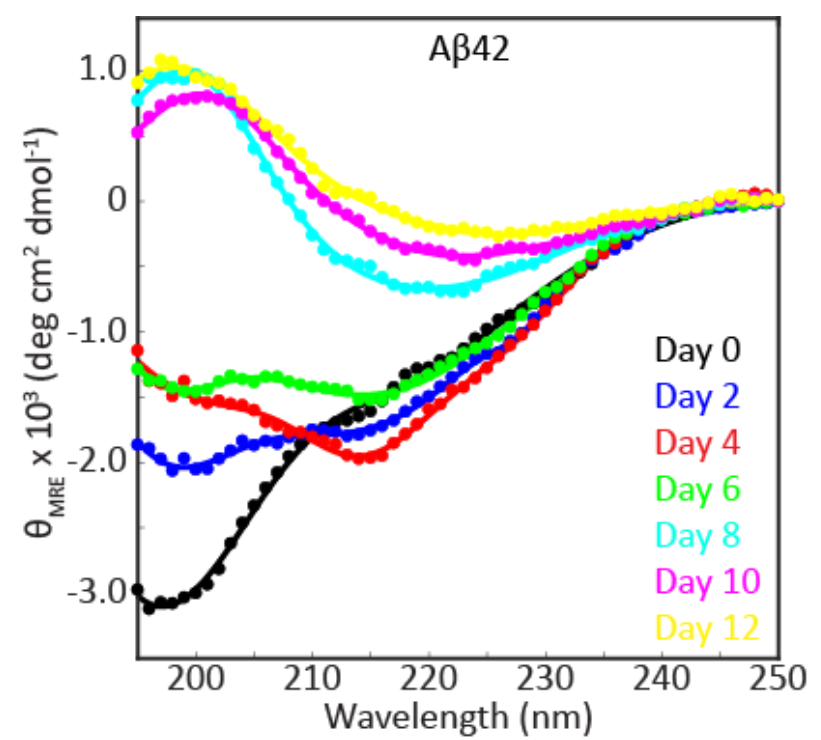

Figure 5.2: CD spectra of AB42 over a 12-day incubation period; Day 0 (black), Day 2 (blue), Day 4 (red), Day 6 (green), Day 8 (cyan), Day 10 (pink), and Day 12 (yellow). 


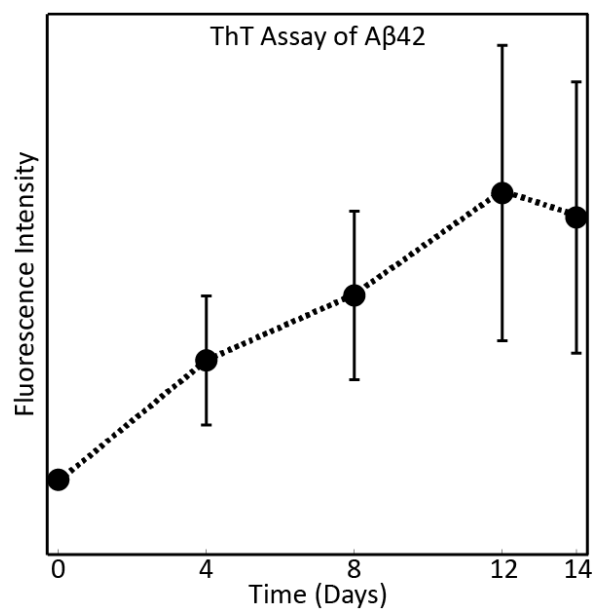

Figure 5.3: ThT fluorescence of AB42 fibrillization over a 14-day incubation period. 


\subsubsection{Nordihydroguaiaretic Acid}

$A \beta$ was incubated at $37^{\circ} \mathrm{C}$ for 6 days. On Day 6 , the polyphenols nordihydroguaiaretic acid, curcumin, and rosmarinic acid were added to aliquots of the $A \beta$ protein solution. Nordihydroguaiaretic acid, having a nonpolar hydrocarbon chain linker, was added to $A \beta$ on Day 6 and the protein remained predominantly $\beta$-sheet structured, as seen in Figure 5.4. However, a small disordered feature with a minimum at $197 \mathrm{~nm}$ is seen, showing that there were initial structural perturbation by the polyphenol on the protein. By Day 8 , a loss in the intensity of the minima at $217 \mathrm{~nm}$ is observed, which continues through Day 12. By Day 12, the protein is almost completely disordered with appearance of the strong minimum at $197 \mathrm{~nm}$ and a significant loss of the minimum at $217 \mathrm{~nm}$. Although, the effects of nordihydroguaiaretic acid are slow, the end result shows that the polyphenol does have the ability to destabilize pre-formed $A \beta$ fibrils.

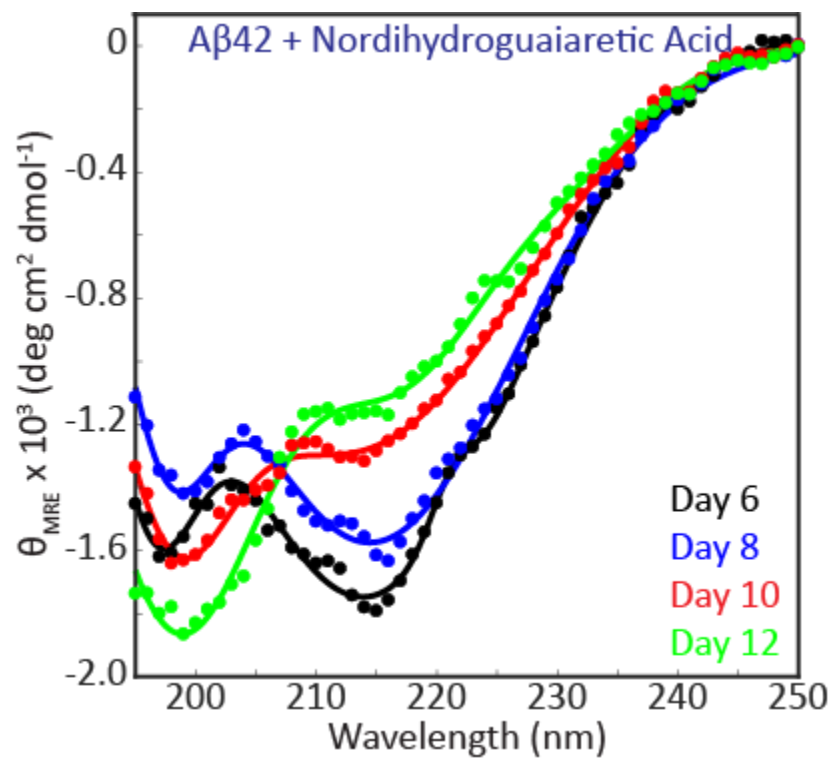

Figure 5.4: CD spectra of AB42 treated with nordihydroguaiaretic acid midway during a 12-day incubation period. The AB protein was treated with nordihydroguaiaretic acid on Day 6 (black). The protein continued to incubate through Day 8 (blue), Day 10 (red), and Day 12 (green). 


\subsubsection{Curcumin}

Curcumin, has a more polar chain linker than nordihydroguaiaretic acid. When curcumin was added to the $A \beta$ sample on Day 6 , the protein's structure stopped changing for the remainder of the incubation period. Additionally, curcumin immediately caused the protein to shift structure from predominantly $\beta$-sheet to partially disordered, as seen in Figure 5.5. Curcumin seems to stabilize the protein in an intermediate state with both disordered and $\beta$-sheet structures. With curcumin, the minimum at $197 \mathrm{~nm}$ indicative of disordered structure is more intense than the minimum at $217 \mathrm{~nm}$ which is indicative of $\beta$-sheet structure, showing that the destabilizing effects of curcumin are strong.

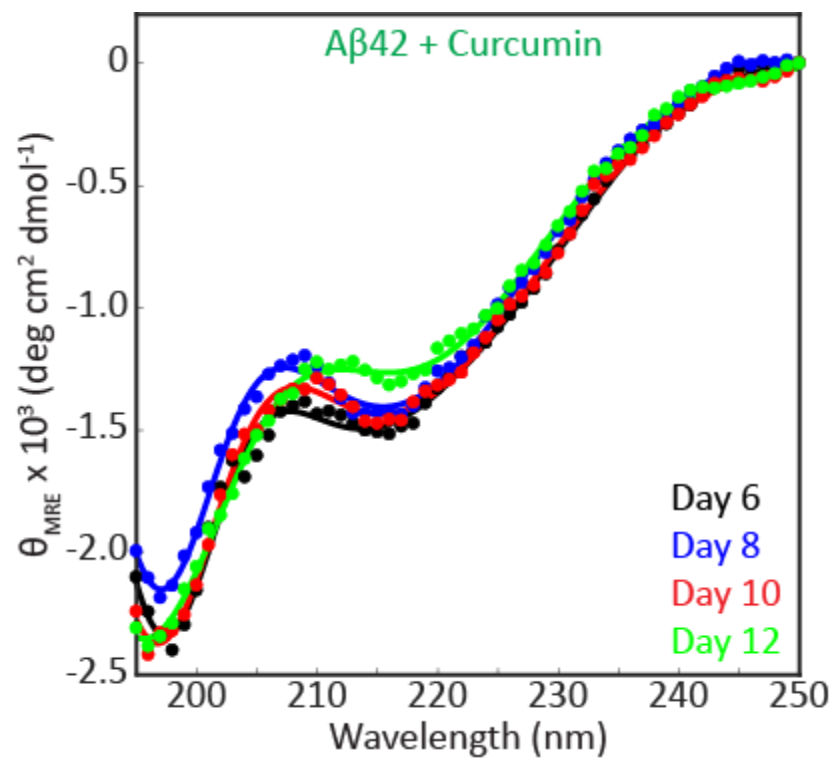

Figure 5.5: $C D$ spectra of $A B 42$ treated with curcumin midway during a 12-day incubation period. The AB protein was treated with curcumin on Day 6 (black). The protein continued to incubate through Day 8 (blue), Day 10 (red), and Day 12 (green). 


\subsubsection{Rosmarinic Acid}

Rosmarinic acid has the most polar chain linker. Again, it was added to the $A \beta$ sample on Day 6 . The structure of the protein immediately shifted toward a more disordered state with the minimum at $197 \mathrm{~nm}$ strengthening, as seen in Figure 5.6.

There was still a slight feature at $217 \mathrm{~nm}$ suggestive of residual $\beta$-sheet structure, however the intensity of this feature reduces at each of the following time points.

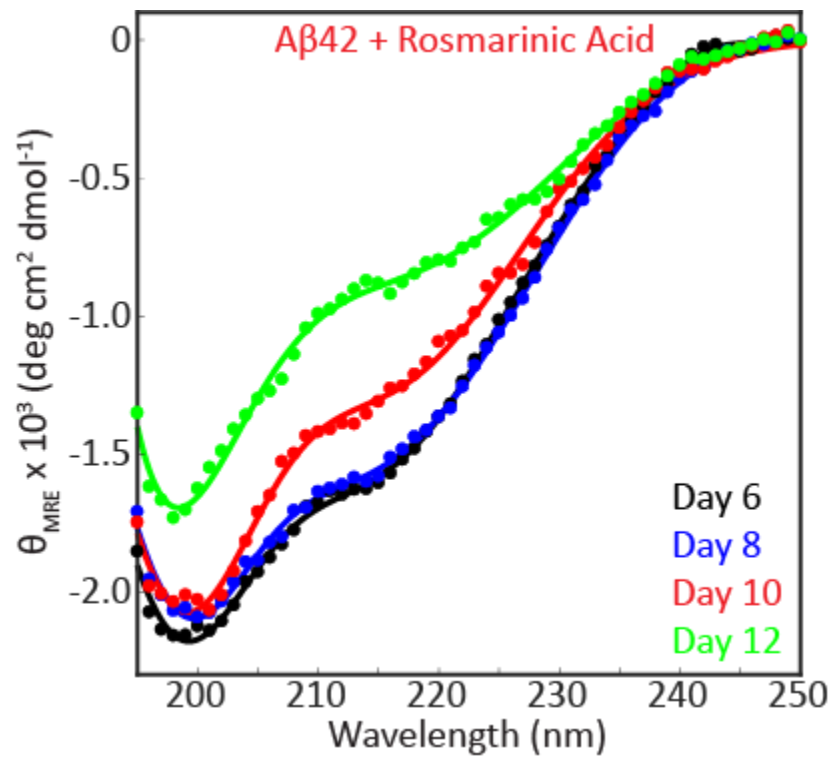

Figure 5.6: CD spectra of AB42 treated with rosmarinic acid midway during a 12-day incubation period. The AB protein was treated with rosmarinic acid on Day 6 (black). The protein continued to incubate through Day 8 (blue), Day 10 (red), and Day 12 (green).

\subsection{Discussion}

Each of the polyphenols tested here caused a shift from $\beta$-sheet to more disordered structure when added to pre-formed fibrils. However, the effect manifested differently for each polyphenol. Interestingly nordihydroguaiaretic acid appeared to initially promote $\beta$-sheet structure and then disrupt it over the remaining 6 days of incubation. Curcumin on the other hand, appeared to freeze $A \beta$ in an intermediate state containing significant amounts of both disordered and $\beta$-sheet structures. Rosmarinic 
acid also shifted the overall structure of $A \beta$ toward disordered structure and continued to do so throughout the experiment. Although there is no clear trend with the increase in the polarity of the chain linker region, all three polyphenols disrupted the fibrillization process. Nordihydroguaiaretic acid and rosmarinic acid continued to disrupt fibrils throughout the remaining incubation period but curcumin appeared to freeze the peptide in an intermediate state. Curcumin has the longest linker region between the phenols, which may be a factor. 


\subsection{References}

1. Lakey-Beitia, J.; Berrocal, R.; Rao, K. S.; Durant, A. A., Polyphenols as therapeutic molecules in Alzheimer's disease through modulating amyloid pathways. Mol Neurobiol 2015, 51 (2), 466-479.

2. Ono, K.; Yoshiike, Y.; Takashima, A.; Hasegawa, K.; Naiki, H.; Yamada, M., Potent antiamyloidogenic and fibril-destabilizing effects of polyphenols in vitro: implications for the prevention and therapeutics of Alzheimer's disease. J Neurochem 2003, 87 (1), 172-181.

3. Shoval, H.; Lichtenberg, D.; Gazit, E., The molecular mechanisms of the anti-amyloid effects of phenols. Amyloid 2007, 14 (1), 73-87.

4. Porat, Y.; Abramowitz, A.; Gazit, E., Inhibition of amyloid fibril formation by polyphenols: structural similarity and aromatic interactions as a common inhibition mechanism. Chem Biol Drug Des 2006, 67 (1), 27-37.

5. Gazit, E., Mechanisms of amyloid fibril self-assembly and inhibition: model short peptides as a key research tool. FEBS J 2005, 272, 5971-5978.

6. Gazit, E., Global analysis of tandem aromatic octapeptide repeats: The significance of the aromatic-glycine motif. Bioinformatics 2002, 18 (6), 880-883.

7. Porat, Y.; Mazor, Y.; Efrat, S.; Gazit, E., Inhibition of islet amyloid polypeptide fibril formation: a potential role for heteroaromatic interactions. Biochem 2004, 43, 14454-14462.

8. Azriel, R.; Gazit, E., Analysis of the minimuml amyloid-forming fragment of the islet amyloid polypeptide. An experimental support for the key role of the phenylalanine residue in amyloid formation. J Biol Chem 2001, 276 (36), 34156-61.

9. Hudson, S. A.; Ecroyd, H.; Dehle, F. C.; Musgrave, I. F.; Carver, J. A., (-)-Epigallocatechin-3-gallate (EGCG) maintains kappa-casein in its pre-fibrillar state without redirecting its aggregation pathway. J Mol Biol 2009, 392 (3), 689-700.

10. Convertino, M.; Pellarin, R.; Catto, M.; Carotti, A.; Caflisch, A., 9,10-Anthraquinone hinders betaaggregation: how does a small molecule interfere with Abeta-peptide amyloid fibrillation? Protein Sci 2009, 18 (4), 792-800. 
11. Lashuel, H. A.; Hartley, D. M.; Balakhaneh, D.; Aggarwal, A.; Teichberg, S.; Callaway, D. J., New class of inhibitors of amyloid-beta fibril formation. Implications for the mechanism of pathogenesis in Alzheimer's disease. J Biol Chem 2002, 277 (45), 42881-90. 


\section{Chapter VI: Conclusions}

The aggregation pathway of the amyloid- $\beta$ protein can be altered by introduction of polyphenolic compounds. This alteration can occur during the formation and extension stages of the aggregation, or during the destabilization stage of the pathway. How a polyphenol behaves when introduced to the protein solution is dependent both on chain linker polarity and number of hydroxyl ring substituents on the polyphenol. This investigation examined the effects of nordihydroguaiaretic acid, curcumin, rosmarinic acid, resveratrol, piceatannol, and diethylstilbestrol on $A \beta$ aggregation and destabilization using circular dichroism and deep ultraviolet resonance Raman spectroscopies.

Increasing the number of ring substitutions was correlated with an increased ability to prevent of the formation of fibrils and also to destabilize pre-formed fibrils. Increasing the hydroxyl group substituents on the polyphenol likely facilitates increased hydrogen bonding required with $A \beta$. The polyphenols diethylstilbestrol, resveratrol, and piceatannol have increasing numbers of hydroxyl substituents on their rings, having two, three, and four respectively. Piceatannol, having the greatest number of hydroxyl substituents has the highest efficacy in preventing formation of fibrils and destabilizing pre-formed fibrils.

Increasing the polarity of the chain linker resulted in decreased stability of the protein during the aggregation process. The nonpolar chain linker of nordihydroguaiaretic acid preserved the disordered structure of the $A \beta$ protein and prevented it from forming $\beta$-sheet structure. Conversely, rosmarinic acid having the 
most polar chain linker was not able to perform in the same manner, increasing the amount of $\beta$-sheet structure in the protein solution. Nordihydroguaiaretic acid, curcumin, and rosmarinic acid were able to disrupt pre-formed $A \beta$ fibrils, each at its own rate, however the final state was similar regardless of added polyphenol. Decreasing the polarity of the chain linker would be optimal in order to prevent, or slow, the formation of fibrils while still having the ability to destabilize pre-formed $A \beta$ fibrils as well.

Future studies including a wider array of polyphenols with varying phenolic ring substitutions and chain linkers is necessary to confirm the results shown here. Amyloid$\beta$ has many unique fragments within its 42 residues which all play a different role in how the protein aggregates. Examples of these fragments include the transmembrane region, extracellular region, glycine zipper motifs, HHQK motif, and KLVFF motif. Investigating how the different structural components of the polyphenols affect each of these $A \beta$ fragments is crucial to gain a full understanding on how they are playing a role in the complete aggregation pathway of $A \beta$. 


\section{VITA}

Brittany L. Hagenhoff was born on August $2^{\text {nd }}, 1990$ in Jefferson City, Missouri where she was raised. She attended the University of Central Missouri where she obtained her B.S. in Chemistry in the Spring of 2012. She received a Ph. D. in Chemistry under the supervision of Dr. Renee D. JiJi at the University of Missouri-Columbia, with a graduation date of Fall 2017. She attained a position as a tenure track analytical faculty member at Truman State University in Kirksville, Missouri beginning in the Fall of 2017. 